LU TP 06-07

HISKP - TH - 05-26

hep-lat/0602003

revised February 2006

\title{
Three-Flavor Partially Quenched Chiral Perturbation Theory at NNLO for Meson Masses and Decay Constants
}

\author{
Johan Bijnens $^{a}$, Niclas Danielsson ${ }^{a, b}$ and Timo A. Lähde ${ }^{c}$ \\ ${ }^{a}$ Department of Theoretical Physics, Lund University, \\ Sölvegatan 14A, SE - 22362 Lund, Sweden \\ ${ }^{b}$ Division of Mathematical Physics, Lund Institute of Technology, Lund University, \\ Box 118, SE - 22100 Lund, Sweden \\ ${ }^{c}$ Helmholtz-Institut für Strahlen- und Kernphysik (HISKP), Bonn University, \\ Nußallee 14-16, D - 53115 Bonn, Germany
}

\begin{abstract}
We discuss Partially Quenched Chiral Perturbation Theory (PQ $\chi \mathrm{PT})$ and possible fitting strategies to Lattice QCD data at next-to-next-to-leading order (NNLO) in the mesonic sector. We also present a complete calculation of the masses of the charged pseudoscalar mesons, in the supersymmetric formulation of PQ $\chi \mathrm{PT}$. Explicit analytical results are given for up to three nondegenerate sea quark flavors, along with the previously unpublished expression for the pseudoscalar meson decay constant for three nondegenerate sea quark flavors. The numerical analysis in this paper demonstrates that the corrections at NNLO are sizable, as expected from earlier work.
\end{abstract}

PACS: 12.38.Gc, 12.39.Fe, 11.30.Rd 


\title{
Three-Flavor Partially Quenched Chiral Perturbation Theory at NNLO for Meson Masses and Decay Constants
}

\author{
Johan Bijnens, ${ }^{1}$ Niclas Danielsson, ${ }^{1,2}$ and Timo A. Lähde ${ }^{3}$ \\ ${ }^{1}$ Department of Theoretical Physics, Lund University, \\ Sölvegatan 14A, SE - 223 62 Lund, Sweden \\ ${ }^{2}$ Division of Mathematical Physics, Lund Institute of Technology, Lund University, \\ Box 118, SE - 22100 Lund, Sweden \\ ${ }^{3}$ Helmholtz-Institut für Strahlen- und Kernphysik (HISKP), Bonn University, \\ Nußallee 14-16, D - 53115 Bonn, Germany
}

\begin{abstract}
We discuss Partially Quenched Chiral Perturbation Theory (PQ $\chi \mathrm{PT})$ and possible fitting strategies to Lattice QCD data at next-to-next-to-leading order (NNLO) in the mesonic sector. We also present a complete calculation of the masses of the charged pseudoscalar mesons, in the supersymmetric formulation of PQ $\chi \mathrm{PT}$. Explicit analytical results are given for up to three nondegenerate sea quark flavors, along with the previously unpublished expression for the pseudoscalar meson decay constant for three nondegenerate sea quark flavors. The numerical analysis in this paper demonstrates that the corrections at NNLO are sizable, as expected from earlier work.
\end{abstract}

PACS numbers: 12.38.Gc, 12.39.Fe, 11.30.Rd

\section{INTRODUCTION}

The generally accepted theory of the strong interaction, Quantum Chromo Dynamics (QCD), provides in principle a way to derive various low-energy hadronic observables, such as masses and decay constants. However, an analytic $a b$ initio derivation of such properties has not yet been possible. An alternative approach called Lattice QCD, where the functional integrals are evaluated using numerical Monte Carlo techniques on a discretized spacetime lattice, suggests a way around this problem, but is nevertheless not free of difficulties of its own. In particular, the properties of low-mass particles are especially difficult to calculate as they can propagate over large distances on the lattice, giving rise to large nonlocal correlations which limit the precision obtainable with given computer resources. As a consequence, most simulations have been performed with heavier quark masses than those of the physical world. The quark masses used in present Lattice QCD simulations typically fulfill $m_{u, d} \geq m_{s} / 8$. The results then have to be extrapolated down to the physical masses of $\sim m_{s} / 25$.

The preferable way to perform the extrapolation to physical quark masses is by means of Chiral Perturbation Theory $(\chi \mathrm{PT})[1,2]$, which provides a theoretically correct description of the low-energy properties of QCD within the range of quark masses where $\chi \mathrm{PT}$ calculations can be considered accurate enough. In particular, the appearance of so-called chiral logarithms renders simple polynomial extrapolation insufficient. If the Lattice QCD simulations are performed with quark masses in the chiral regime, i.e. with quark masses for which $\chi \mathrm{PT}$ calculations are reliable, then they can be used to e.g. determine the effective low-energy constants (LEC:s) of $\chi$ PT. Nevertheless, in practice it is still difficult to reach the chiral regime and therefore many large scale lattice simulations of so-called quenched QCD have been per- formed, in which the effects of closed (sea) quark loops are neglected. Consideration of such loop effects requires repeated evaluation of fermion determinants, which is computationally extremely expensive. It should be noted that first Lattice QCD calculations with sea quark masses significantly below $m_{s}$ are now becoming available.

From a computational point of view, it is however much cheaper to vary the valence quark masses $m_{\text {val }}$ rather than those of the sea quarks, $m_{\text {sea }}$. Therefore it is not uncommon to perform simulations where the sea quark loops are not neglected, but rather suppressed by choosing $m_{\text {sea }}>m_{\text {val }}$, or even $n_{\text {sea }} \neq n_{\text {val }}$, where $n_{\text {val }}$ and $n_{\text {sea }}$ denote the number of each quark species in the theory. Such procedures are referred to as partial quenching and lead into a space of unphysical theories.

\section{A. Partially Quenched Theories}

At first, one might expect that partially quenched (PQ) theories could yield only qualitative information about QCD. However, since unquenched QCD may be recovered from partially quenched QCD (PQQCD) in the limit of equal sea and valence quark masses, it follows that QCD and PQQCD are continuously connected by variation of sea quark masses. In contrast, this is not true for fully quenched QCD. Nevertheless, $\chi \mathrm{PT}$ can be extended to the case of quenched QCD, which was done by Bernard, Golterman and Sharpe [3, 4] after earlier work by Morel [5]. The extension to the partially quenched case was done in Ref. 6]. A more extensive discussion can be found in the work of Sharpe and Shoresh [7, 8].

The formulation of Partially Quenched Chiral Perturbation Theory $(\mathrm{PQ} \chi \mathrm{PT})$ is such that the dependence on the sea quark masses is explicit, and thus the limit of equal sea and valence quark masses can also be considered for $\mathrm{PQ} \chi \mathrm{PT}$. It follows that $\chi \mathrm{PT}$ is recovered as a continuous limit of $\mathrm{PQ} \chi \mathrm{PT}$ just as QCD is from PQQCD. 
In particular, the LEC:s of $\chi \mathrm{PT}$, which are of physical significance, can be obtained directly from those of $\mathrm{PQ} \chi \mathrm{PT}$. In addition, the ability to vary valence and sea quark masses separately allows more information to be extracted at fewer values of the sea quark masses. This is another reason to consider PQQCD in lattice simulations as discussed in detail in Ref. 7].

This paper is devoted to the extension of $\mathrm{PQ} \chi \mathrm{PT}$ to next-to-next-to-leading-order (NNLO). The discussions already given in Refs. [9, 10, 11] are completed and the calculations of the masses and decay constants of the charged, or off-diagonal, pseudoscalar mesons are completed for the case of $n_{\text {sea }}=3$. It should be noted that indications already exist, by the $[\mathrm{qq}+\mathrm{q}]$ collaboration 12 and the MILC collaboration 13], that the $\mathcal{O}\left(p^{6}\right)$ contributions to these quantities are sizable, and that the inclusion of such effects can have a significant impact on the chiral extrapolations down to the physical quark masses. More examples can be found in the proceedings of the Lattice 2005 conference.

\section{B. Partially Quenched $\chi \mathbf{P T}$ at NNLO}

The meson masses and decay constants of the pseudoscalar mesons in PQ $\chi \mathrm{PT}$ for $n_{\text {sea }}=3$ to NLO, one loop, or $\mathcal{O}\left(p^{4}\right)$ in the momentum expansion, were calculated in Refs. 6, 7, 8]. First results for these quantities at NNLO, two loops, or $\mathcal{O}\left(p^{6}\right)$, namely the mass of a charged pseudoscalar meson for degenerate sea and degenerate valence quark masses, can be found in Ref. [9]. The NNLO expression for the decay constant of a charged pseudoscalar meson with two nondegenerate sea quarks has been calculated in Ref. [10]. The full results for pseudoscalar meson masses and decay constants for $n_{\text {sea }}=2$ were given in Ref. [11]. This paper presents the full nondegenerate calculations of the charged pseudoscalar meson mass to NNLO for $n_{\text {sea }}=3$, along with the calculation of the decay constant for three nondegenerate sea quarks.

In general, the NNLO expressions are very long, but it is possible to shorten them considerably by introducing a specialized notation which can accommodate the complications inherent in $\mathrm{PQ} \chi \mathrm{PT}$. Furthermore, this notation satisfies many different algebraic relations, which allows for a systematic simplification to be carried out. In the next sections, the technical background for the NNLO calculations, together with the above-mentioned notation is presented, followed by the results for the NNLO masses and decay constants of the charged pseudoscalar mesons, and a numerical analysis. All analytical formulas are given explicitly, but they can also be downloaded from the website [14].

This paper is organized in the following manner: Sect. III introduces PQ $\chi \mathrm{PT}$ and discusses the various aspects relevant for calculations at NNLO. In particular, the relations between the various sets of LEC:s are highlighted. This section also includes an overview of all no- tation used for loop integrals and combinations of quark masses. Sects. III and IV] contain the analytical NNLO expressions for the masses and decay constants of the charged pseudoscalar mesons. Sect. $\mathbb{\nabla}$ presents a discussion of the checks performed on the analytical and numerical calculations, a numerical analysis of the results, and an elaboration on the extraction of the various LEC:s from Lattice QCD calculations is given in Sect. $\mathrm{VE}$ Our conclusions are shortly discussed in Sect. VI]

\section{TECHNICAL ASPECTS OF PQ $\chi$ PT}

The technical aspects of $\mathrm{PQ} \chi \mathrm{PT}$ calculations to NLO have been thoroughly covered, for the supersymmetric formulation of PQ $\chi \mathrm{PT}$, in Refs. [7, 8]. The new parts specific to the NNLO calculations have been covered briefly in Refs. 9, 10, 11]. The aim of this section is to collect all this information in one place and describe the NNLO aspects in somewhat greater detail.

\section{A. Supersymmetric $\mathbf{P Q} \chi \mathbf{P T}$}

Let us first recall a few aspects of $\chi \mathrm{PT}$, and note in passing that introductions to the subject can be found e.g. in Ref. [15]. For $n_{f}$ massless quark flavors, QCD has a chiral symmetry

$$
G=S U\left(n_{f}\right)_{L} \times S U\left(n_{f}\right)_{R},
$$

which is spontaneously broken by the vacuum to its diagonal subgroup $H=S U\left(n_{f}\right)_{V}$, where $V=L+R$. The Goldstone bosons produced by this spontaneous breakdown live on the coset $G / H$ which is also an $S U\left(n_{f}\right)$ manifold and can be parameterized in terms of a special unitary matrix $U$. This matrix is conventionally written in terms of a traceless Hermitian matrix according to

$$
U \equiv \exp (i \sqrt{2} \phi / F)
$$

where the constant $F$ with dimension of energy can be shown to be related to the decay constant of the pion. For three quark flavors $(u, d, s)$, the matrix $\phi$ has a flavor structure

$$
\phi=\left(\begin{array}{ccc}
u \bar{u} & u \bar{d} & u \bar{s} \\
d \bar{u} & d \bar{d} & d \bar{s} \\
s \bar{u} & s \bar{d} & s \bar{s}
\end{array}\right) .
$$

The above considerations do not distinguish between valence and sea quarks whereas the construction of a PQ theory requires a mechanism which gives different masses to sea quarks and valence quarks. This effect may be produced in a systematical way by adding to $\chi \mathrm{PT}$ explicit sea quarks as well as unphysical bosonic ghost quarks. The latter cancel exactly all effects of closed valence quark loops due to their different statistics, provided that their masses are identical to those of the valence quarks. 
Supersymmetric $\mathrm{PQ} \chi \mathrm{PT}$ thus contains a set of fermionic as well as bosonic quarks. This leads to a modification of the chiral symmetry group as given by Eq. (1), which in the PQ theory has the graded structure

$$
G=S U\left(n_{\mathrm{val}}+n_{\text {sea }} \mid n_{\mathrm{val}}\right)_{L} \times S U\left(n_{\mathrm{val}}+n_{\mathrm{sea}} \mid n_{\mathrm{val}}\right)_{R} .
$$

The precise structure of $G$ is somewhat different as discussed in [7, 8]. This theory contains $n_{\text {val }}$ valence and $n_{\text {val }}$ ghost quarks, as well as $n_{\text {sea }}$ flavors of sea quarks. As the PQ theories include bosonic ghost quarks, they are not normal relativistic quantum field theories since they violate the spin-statistics theorem. However, under the assumption that the low-energy structure of such a theory can be described similarly to the case of normal $\mathrm{QCD}$, one arrives at an effective low-energy theory in terms of a matrix $U$, according to

$$
U \equiv \exp (i \sqrt{2} \Phi / \hat{F})
$$

where the matrix $\Phi$ now has a more complicated flavor structure than $\phi$ in Eq. (3) because of the different types of quarks present. In terms of a sub-matrix notation for the flavor structure

$$
q_{a} \bar{q}_{b}=\left(\begin{array}{ccc}
u_{a} \bar{u}_{b} & u_{a} \bar{d}_{b} & u_{a} \bar{s}_{b} \\
d_{a} \bar{u}_{b} & d_{a} \bar{d}_{b} & d_{a} \bar{s}_{b} \\
s_{a} \bar{u}_{b} & s_{a} \bar{d}_{b} & s_{a} \bar{s}_{b}
\end{array}\right),
$$

the matrix $\Phi$ becomes

$$
\Phi=\left(\begin{array}{ccc}
{\left[q_{V} \bar{q}_{V}\right]} & {\left[q_{V} \bar{q}_{S}\right]} & {\left[q_{V} \bar{q}_{B}\right]} \\
{\left[q_{S} \bar{q}_{V}\right]} & {\left[q_{S} \bar{q}_{S}\right]} & {\left[q_{S} \bar{q}_{B}\right]} \\
{\left[q_{B} \bar{q}_{V}\right]} & {\left[q_{B} \bar{q}_{S}\right]} & {\left[q_{B} \bar{q}_{B}\right]}
\end{array}\right),
$$

where the labels $V, S$ and $B$ stand for valence, sea and bosonic quarks, respectively. The size of each sub-matrix depends on the exact number of quark flavors used.

The quarks $q_{V}, q_{S}$ and their respective antiquarks are fermions, while the quarks $q_{B}$ and their antiquarks are bosons. Thus a combination of a fermionic quark and a bosonic quark yield a fermionic (anticommuting) field, while a combination of two fermionic or two bosonic quarks result in a bosonic field. Each sub-matrix in Eq. (7) therefore consists of either fermionic of bosonic fields only. Although in principle arbitrary, the bosonic quarks are given the same masses as the corresponding valence quarks in order to cancel the contributions from closed valence quark loops. The above formalism is often referred to as supersymmetric $\mathrm{PQ} \chi \mathrm{PT}$, although this only refers to the graded group structure of the matrices used in the construction of the theory. Furthermore, the term 'bosonic' merely indicates that those quark terms are treated as commuting variables. They are still spin $1 / 2$ particles since the quark-antiquark pairs should build up particles with spin 0 , representing the mesons of the theory. This violation of the spin-statistics theorem implies, as stated above, that in general the PQ theory is not a (fully causal) field theory.

In the version of $\mathrm{PQ} \chi \mathrm{PT}$ used in this paper, the singlet $\Phi_{0}$ field has been dropped as discussed in Ref. [8]. This PQ singlet is expected to be heavy due to the axial anomaly in the same way as the $\eta^{\prime}$ which is not included in unquenched $\chi \mathrm{PT}$. Having defined the supersymmetric field matrix $\Phi$ in Eq. (7), one then proceeds by constructing an effective Lagrangian for the PQ theory by requiring that this Lagrangian should be invariant under $S U\left(n_{\text {val }}+n_{\text {sea }} \mid n_{\text {val }}\right)_{L} \times S U\left(n_{\text {val }}+n_{\text {sea }} \mid n_{\text {val }}\right)_{R}$ in the same way as the Lagrangian of $\chi \mathrm{PT}$ was required to be invariant under $S U\left(n_{f}\right)_{L} \times S U\left(n_{f}\right)_{R}$. This modification results in the same Lagrangian structure as for $\chi \mathrm{PT}$, provided that the traces of matrix products in those Lagrangians are replaced by supertraces. The supertraces are defined in terms of ordinary traces by

$$
\operatorname{Str}\left(\begin{array}{ll}
A & B \\
C & D
\end{array}\right)=\operatorname{Tr} A-\operatorname{Tr} D
$$

where $A, B, C$ and $D$ denote block matrices. For example, the block $B$ corresponds to the $\left[q_{V} \bar{q}_{B}\right]$ and $\left[q_{S} \bar{q}_{B}\right]$ sectors of the field matrix in Eq. (17) and contains anticommuting fields, while the block $D$ represents the $\left[q_{B} \bar{q}_{B}\right]$ sector of Eq. (7). Note that the removal of $\Phi_{0}$ implies the assumption

$$
\operatorname{Str}(\Phi)=0 .
$$

The entire external field formalism introduced by Gasser and Leutwyler 2] for $\chi \mathrm{PT}$ can be generalized to include the extra degrees of freedom. In practice, external fields will be used in the valence sector only.

\section{B. Lagrangians and LECs}

We now proceed with the construction of the Lagrangians for the Goldstone bosons and organize them according to the Weinberg power counting scheme. The external fields included are the vector and axial-vector fields $v_{\mu}$ and $a_{\mu}$, as well as the scalar and pseudo-scalar external sources $s$ and $p$. These are the suitably generalized versions of those used in standard $\chi \mathrm{PT}$ [2]. Under a symmetry transformation $g_{L, R} \in S U\left(n_{\text {val }}+n_{\text {sea }} \mid n_{\text {val }}\right)_{L, R}$ the fields transform as

$$
\begin{aligned}
U & \rightarrow g_{R} U g_{L}^{\dagger}, \\
\chi \equiv 2 \hat{B}(s+i p) & \rightarrow g_{R} \chi g_{L}^{\dagger}, \\
l_{\mu} \equiv v_{\mu}-a_{\mu} & \rightarrow g_{L} l_{\mu} g_{L}^{\dagger}-i \partial_{\mu} g_{L} g_{L}^{\dagger}, \\
r_{\mu} \equiv v_{\mu}+a_{\mu} & \rightarrow g_{R} r_{\mu} g_{R}^{\dagger}-i \partial_{\mu} g_{R} g_{R}^{\dagger} .
\end{aligned}
$$

However, one can also define another set of quantities which behave differently under chiral symmetry transformations, and which turn out to be more useful for the construction of the Lagrangians. If one considers

$$
u \equiv \exp (i \Phi /(\sqrt{2} \hat{F})),
$$


then it is possible to find a matrix $h$ such that

$$
u \rightarrow g_{R} u h^{\dagger}=h u g_{L}^{\dagger} .
$$

One can then proceed by constructing a set of quantities that transform under chiral symmetry as $X \rightarrow h X h^{\dagger}$. Such quantities are

$$
\begin{aligned}
u_{\mu} & =i\left\{u^{\dagger}\left(\partial_{\mu}-i r_{\mu}\right) u-u\left(\partial_{\mu}-i l_{\mu}\right) u^{\dagger}\right\} \\
\chi_{ \pm} & =u^{\dagger} \chi u^{\dagger} \pm u \chi^{\dagger} u \\
f_{ \pm}^{\mu \nu} & =u F_{L}^{\mu \nu} u^{\dagger} \pm u^{\dagger} F_{R}^{\mu \nu} u
\end{aligned}
$$

where $F_{L}$ and $F_{R}$ denote the field strengths of the external fields $l$ and $r$, such that $F_{L}^{\mu \nu}=\partial^{\mu} l^{\nu}-\partial^{\nu} l^{\mu}-i\left[l^{\mu}, l^{\nu}\right.$. $F_{R}^{\mu \nu}$ is then defined analogously in terms of $r$. It should be noted that the Minkowski convention is used throughout this paper instead of the Euclidean one needed in lattice QCD, in order to maintain compatibility with the existing literature in NNLO $\chi \mathrm{PT}$. In terms of the quantities defined in Eq. (13) the lowest order, or $\mathcal{O}\left(p^{2}\right)$ Lagrangian is given by

$$
\mathcal{L}_{2}=\frac{\hat{F}^{2}}{4}\left\langle u^{\mu} u_{\mu}+\chi_{+}\right\rangle,
$$

where the shorthand notation

$$
\langle A\rangle=\operatorname{Str} A
$$

has been introduced. At this level there are two parameters $\hat{F}$ and $\hat{B}$, which depend on the number of sea quark flavors in the PQ theory. In the power counting scheme, each derivative or factor of $l_{\mu}, r_{\mu}$ counts as one, and each factor of $s, p$ as two powers of the momentum $p$. The order in $p$ is indicated by the subscript of the Lagrangian. The $\mathcal{O}\left(p^{4}\right)$ Lagrangian has the generic form [2]

$$
\begin{aligned}
\mathcal{L}_{4} & =\sum_{i=0}^{12} \hat{L}_{i} X_{i}+\text { contact terms } \\
& =\hat{L}_{0}\left\langle u^{\mu} u^{\nu} u_{\mu} u_{\nu}\right\rangle+\hat{L}_{1}\left\langle u^{\mu} u_{\mu}\right\rangle^{2}+\hat{L}_{2}\left\langle u^{\mu} u^{\nu}\right\rangle\left\langle u_{\mu} u_{\nu}\right\rangle \\
& +\hat{L}_{3}\left\langle\left(u^{\mu} u_{\mu}\right)^{2}\right\rangle+\hat{L}_{4}\left\langle u^{\mu} u_{\mu}\right\rangle\left\langle\chi_{+}\right\rangle+\hat{L}_{5}\left\langle u^{\mu} u_{\mu} \chi_{+}\right\rangle \\
& +\hat{L}_{6}\left\langle\chi_{+}\right\rangle^{2}+\hat{L}_{7}\left\langle\chi_{-}\right\rangle^{2}+\frac{\hat{L}_{8}}{2}\left\langle\chi_{+}^{2}+\chi_{-}^{2}\right\rangle \\
& -i \hat{L}_{9}\left\langle f_{+}^{\mu \nu} u_{\mu} u_{\nu}\right\rangle+\frac{\hat{L}_{10}}{4}\left\langle f_{+}^{2}-f_{-}^{2}\right\rangle \\
& +i \hat{L}_{11}\left\langle\hat{\chi}_{-}\left(\nabla^{\mu} u_{\mu}-\frac{i}{2} \hat{\chi}_{-}\right)\right\rangle \\
& +\hat{L}_{12}\left\langle\left(\nabla^{\mu} u_{\mu}-\frac{i}{2} \hat{\chi}_{-}\right)^{2}\right\rangle \\
& +\hat{H}_{1}\left\langle F_{L}^{2}+F_{R}^{2}\right\rangle+\hat{H}_{2}\left\langle\chi \chi^{\dagger}\right\rangle
\end{aligned}
$$

where the definition $\hat{\chi}_{-} \equiv \chi_{-}-\left\langle\chi_{-}\right\rangle / n_{\text {sea }}$ has been applied. Furthermore, the lowest order equation of motion is given by

$$
\nabla^{\mu} u_{\mu}-\frac{i}{2} \hat{\chi}_{-}=0
$$

The Lagrangian of Eq. (16) contains three types of terms. Of these, the terms proportional to $\hat{H}_{i}$ are contact terms which contain external fields only. Thus they are not relevant for low-energy phenomenology, but they are necessary for the computation of operator expectation values. Their values are determined by the precise definition used for the QCD currents, and they are conventionally labeled $h_{i}^{r}$ and $H_{i}^{r}$ for unquenched $\chi \mathrm{PT}$ with $n_{f}=2$ and $n_{f}=3$ quark flavors, respectively. The terms that depend on $\hat{L}_{11}$ and $\hat{L}_{12}$ are proportional to the equations of motion, and as such they can always be reabsorbed in higher order LEC:s, see Ref. [16] for a full proof. The Lagrangian at $\mathcal{O}\left(p^{6}\right)$ is also known [17] and can be written in the form

$$
\mathcal{L}_{6}=\sum_{i=1}^{112} \hat{K}_{i} Y_{i}+\text { contact terms, }
$$

where the form of the operators $Y_{i}$ can be found in Refs. 16, 17]. The PQ $\chi \mathrm{PT}$ operators may again be obtained from the results of Refs. 2, 16, 17] if all traces are replaced by supertraces. All manipulations and identities used there to decrease the number of terms in the $\mathcal{O}\left(p^{6}\right)$ Lagrangian, such as integration by parts and usage of the equation of motion, remain valid under this modification. This is why it is possible to apply the $n_{f}$ flavor results of Refs. 2, 16, 17] to $\mathrm{PQ} \chi \mathrm{PT}$ by using the appropriate graded matrices and supertraces.

\begin{tabular}{|c|c|c|c|c|c|}
\hline$n_{f}, n_{\text {sea }}$ & $\begin{array}{c}\chi \mathrm{PT} \\
2\end{array}$ & $\begin{array}{c}\chi \mathrm{PT} \\
3\end{array}$ & $\begin{array}{c}\chi \mathrm{PT} \\
n\end{array}$ & $\begin{array}{c}\mathrm{PQ} \chi \mathrm{PT} \\
2\end{array}$ & $\begin{array}{c}\mathrm{PQ} \chi \mathrm{PT} \\
3\end{array}$ \\
\hline LO & $F, B$ & $F_{0}, B_{0}$ & $F_{0}^{(n)}, B_{0}^{(n)}$ & $F, B$ & $F_{0}, B_{0}$ \\
\hline NLO & $l_{i}^{r}$ & $L_{i}^{r}$ & $L_{i}^{r(n)}$ & $L_{i}^{r(2 p q)}$ & $L_{i}^{r(3 p q)}$ \\
\hline$n_{\mathrm{ph}}+n_{\mathrm{ct}}$ & $7+3$ & $10+2$ & $11+2$ & $11+2$ & $11+2$ \\
\hline & $c_{i}^{r}$ & $C_{i}^{r}$ & & $K_{i}^{r(2 p q)}$ & $K_{i}^{r(3 p q)}$ \\
\hline$n_{\mathrm{ph}}+n_{\mathrm{ct}}$ & $53+4$ & $90+4$ & $112+3$ & $112+3$ & $112+3$ \\
\hline
\end{tabular}

The divergences of $\mathrm{PQ} \chi \mathrm{PT}$ follow directly from those of $n_{f}$ flavor $\chi \mathrm{PT}$ calculated in Ref. 16], provided that $n_{f}$ is set equal to the number of sea-quark flavors. This is so since all the manipulations in Ref. [16] for $n_{f}$ flavors remain valid when traces are replaced with supertraces. Alternatively, this derivation of the Lagrangians of PQ $\chi \mathrm{PT}$ can be argued for with the Replica method of Ref. [18]. The renormalization procedure is thus identical with the one of $\chi \mathrm{PT}$ also in the partially quenched case. An extensive discussion of this procedure can be found in

TABLE I: The different sets of LEC:s for unquenched $\left(n_{f}\right)$ and partially quenched $\left(n_{\text {sea }}\right) \chi \mathrm{PT}$. The number of physically relevant terms in each set is indicated by $n_{\mathrm{ph}}$, and the number of contact terms by $n_{\mathrm{ct}}$. The relationships between the various LEC:s are discussed in the text. 
Ref. [16] and references therein. The finite parts of the $\hat{L}_{i}$ and $\hat{K}_{i}$ for the different theories considered are summarized in Table \ For the case of $\chi \mathrm{PT}$ with two or three flavors the generic Lagrangians referred to above can be further simplified using the Cayley-Hamilton relations for $2 \times 2$ or $3 \times 3$ matrices. These allow for a further reduction of the number of operators, resulting in the different sets of constants shown in Table The superscript $r$ for the NLO and NNLO LEC:s indicates that they are the renormalized versions as defined in Ref. 16. In particular, the NNLO coefficients are given by the finite parts of the $\hat{K}_{i}$, multiplied by $\hat{F}^{2}$ in order to obtain dimensionless quantities.

Consider next the LEC:s of PQ $\chi$ PT with $n$ flavors of sea-quarks, which to lowest order are identical to those of unquenched $n$ flavor $\chi \mathrm{PT}$. At NLO, they are $L_{0}^{r(n p q)}$ through $L_{12}^{r(n p q)}$, but as argued above the two terms $L_{11}^{r(n p q)}$ and $L_{12}^{r(n p q)}$ can be removed using field redefinitions or the equations of motion [16, 17]. For three flavors of sea-quarks, the standard choice is

$$
L_{11}^{r(3 p q)}=L_{12}^{r(3 p q)}=0,
$$

whereas the unquenched two-flavor Lagrangian as defined in the first paper of Ref. [2] differs by an $L_{11}^{r}$ type term. This makes no difference at NLO since that term does not contribute, but in order to get the correct correspondence at NNLO between the $l_{i}^{r}, c_{i}^{r}$ and the $L_{i}^{r(2 p q)}, K_{i}^{r(2 p q)}$ one should take

$$
L_{11}^{r(2 p q)}=-l_{4}^{r} / 4, \quad L_{12}^{r(2 p q)}=0 .
$$

For the case of three sea-quarks, the $L_{i}^{r}$ are simple linear combinations of the $L_{i}^{r(3 p q)}$, which have been given in Refs. [10, 17]. Explicitly,

$$
\begin{aligned}
& L_{1}^{r}=L_{1}^{r(3 p q)}+L_{0}^{r(3 p q)} / 2, \\
& L_{2}^{r}=L_{2}^{r(3 p q)}+L_{0}^{r(3 p q)}, \\
& L_{3}^{r}=L_{3}^{r(3 p q)}-2 L_{0}^{r(3 p q)},
\end{aligned}
$$

and

$$
L_{4 \ldots 12}^{r}=L_{4 \ldots 12}^{r(3 p q)},
$$

which indicates, as also discussed in Ref. 19], that $L_{0}^{r(3 p q)}$ is an independent LEC in partially quenched $\chi \mathrm{PT}$ with $n_{\text {sea }}=3$, but in unquenched $\chi \mathrm{PT}$ it can be absorbed into $L_{1}^{r}, L_{2}^{r}$ and $L_{3}^{r}$. One can therefore conclude that the numerical value of $L_{0}^{r(3 p q)}$ cannot be determined by experiment, but is accessible via partially quenched Lattice QCD simulations. Similarly at NNLO, the $C_{i}^{r}$ of unquenched $\chi \mathrm{PT}$ are linear combinations of the $K_{i}^{r(3 p q)}$, and the corresponding relations have been derived in Ref. 17].

The expressions given in the following sections of this paper concern PQ $\chi \mathrm{PT}$ with $n_{\text {sea }}=3$ only, and therefore the superscripts (3pq) of the $L_{i}^{r(3 p q)}$ and the $K_{i}^{r(3 p q)}$ have been suppressed in most of the remaining equations.

\section{Quark Masses and Propagators}

The version of $\mathrm{PQ} \chi \mathrm{PT}$ considered in this paper has three flavors of valence quarks $\left(n_{\mathrm{val}}=3\right)$, three flavors of sea quarks $\left(n_{\text {sea }}=3\right)$ and consequently three flavors of bosonic 'ghost' quarks. For simplicity, the different quark masses are identified in the following calculations by the flavor indices $i=1, \ldots, 9$, rather than by the indices $u, d, s$ and $V, S, B$ of Eqs. (6) and (7). The results are expressed in terms of the quark masses $m_{q}$ via the quantities $\chi_{i}=2 B_{0} m_{q i}$ such that $\chi_{1}, \chi_{2}, \chi_{3}$, belong to the valence sector, $\chi_{4}, \chi_{5}, \chi_{6}$ to the sea sector, and $\chi_{7}, \chi_{8}, \chi_{9}$ to the ghost sector. The latter ones do not appear in the results since the ghost quark masses are always set equal to the masses of the corresponding valence quarks, such that $\chi_{7}=\chi_{1}, \chi_{8}=\chi_{2}$ and $\chi_{9}=\chi_{3}$. As discussed above, this is necessary in order to cancel the disconnected valence quark loops, which are replaced by the shifted mass contributions that involve the sea quarks.

Furthermore, the quantities $d_{\text {val }}$ and $d_{\text {sea }}$ are used to indicate the number of nondegenerate quark masses in each sector. Thus $d_{\mathrm{val}}=1$ has all valence quark masses degenerate, such that $\chi_{1}=\chi_{2}=\chi_{3}$, while $d_{\mathrm{val}}=2$ indicates that $\chi_{1}=\chi_{2} \neq \chi_{3}$. Finally, in the case of $d_{\mathrm{val}}=3$, which is not needed in the present calculations, $\chi_{1} \neq \chi_{2} \neq \chi_{3}$. In a similar way, for the sea quark masses $d_{\text {sea }}=1$ implies that $\chi_{4}=\chi_{5}=\chi_{6}$, while for $d_{\text {sea }}=2$ one has $\chi_{4}=\chi_{5} \neq \chi_{6}$, and for $d_{\text {sea }}=3$ all the sea quark masses are nondegenerate, such that $\chi_{4} \neq \chi_{5} \neq$ $\chi_{6}$. The notation $\left(d_{\text {val }}+d_{\text {sea }}\right)$ is often used in this paper to indicate what degree of degeneracy in the quark masses is being considered. For example, the expression for the pseudoscalar meson mass for $n_{\text {val }}=2$ and $n_{\text {sea }}=3$ in the $(1+2)$ case depends on one (degenerate) valence quark mass and two distinct (nondegenerate) sea quark masses.

The meson propagators for the supersymmetric formulation of PQ $\chi \mathrm{PT}$ have been calculated in Ref. [8], and they correspond to the limit $m_{0} \rightarrow \infty$ of the results in Ref. [3], where $m_{0}$ is the mass parameter of the singlet field $\Phi_{0}$. Again, for calculational reasons, the results of Ref. [8] have been translated from the Euclidean formalism into Minkowski space. In general, three distinct types of propagators are encountered in the calculations. The simplest one is the propagator of a charged, or flavor-offdiagonal meson, connecting the meson field $q_{i} \bar{q}_{j}$ with its antifield $q_{j} \bar{q}_{i}$. It is given by $[8]$

$$
-i G_{i j}^{c}(k)=\frac{\epsilon_{j}}{k^{2}-\chi_{i j}+i \varepsilon} \quad(i \neq j) .
$$

where the combination of quark masses $\chi_{i j} \equiv\left(\chi_{i}+\chi_{j}\right) / 2$ corresponds to the lowest order meson masses, and the signature vector $\epsilon_{j}$ is defined as +1 for the flavor indices of the $n_{\text {val }}+n_{\text {sea }}$ fermionic quarks, and as -1 for the flavor indices of the $n_{\mathrm{val}}$ bosonic ghost quarks. In the present calculation, with the number of valence and sea 
quarks as given above, $\epsilon_{j}$ assumes the values

$$
\epsilon_{j}=\left\{\begin{array}{l}
+1 \text { for } j=1, \ldots, 6 \\
-1 \text { for } j=7,8,9
\end{array}\right.
$$

The propagator of a neutral, or flavor-diagonal meson, has a more complicated structure, since it connects mesons with different flavor indices as well. A propagator which connects the meson fields $q_{i} \bar{q}_{i}$ and $q_{j} \bar{q}_{j}$ is written in the form [8]

$$
G_{i j}^{n}(k)=G_{i j}^{c}(k) \delta_{i j}-G_{i j}^{q}(k) / n_{\text {sea }},
$$

where the second term $G_{i j}^{q}(k)$ is expressed in terms of a sum of single and double poles [7, [8]. There are two distinct cases, as the double pole only appears if either $i=j$ or $\chi_{i}=\chi_{j}$. For $i \neq j$ and $\chi_{i} \neq \chi_{j}$, the propagator $G_{i j}^{q}$ is given by

$$
\begin{aligned}
-i G_{i j}^{q}(k) & =\frac{R_{j \pi \eta}^{i}}{k^{2}-\chi_{i}+i \varepsilon}+\frac{R_{i \pi \eta}^{j}}{k^{2}-\chi_{j}+i \varepsilon} \\
& +\frac{R_{\eta i j}^{\pi}}{k^{2}-\chi_{\pi}+i \varepsilon}+\frac{R_{\pi i j}^{\eta}}{k^{2}-\chi_{\eta}+i \varepsilon}
\end{aligned}
$$

where the factors $R$ are referred to collectively as the single pole residues. For $i=j$ or $\chi_{i}=\chi_{j}$, the residues of the first two terms in eq. (26) become singular and the whole expression should be replaced by

$$
\begin{aligned}
-i G_{i j}^{q}(k) & =\frac{R_{i}^{d}}{\left(k^{2}-\chi_{i}+i \varepsilon\right)^{2}}+\frac{R_{i}^{c}}{k^{2}-\chi_{i}+i \varepsilon} \\
& +\frac{R_{\eta i i}^{\pi}}{k^{2}-\chi_{\pi}+i \varepsilon}+\frac{R_{\pi i i}^{\eta}}{k^{2}-\chi_{\eta}+i \varepsilon},
\end{aligned}
$$

where the residue of the double pole is denoted $R_{i}^{d}$. Note also the appearance of an auxiliary residue $R_{i}^{c}$ in eq. (27). All expressions for the propagator $G_{i j}^{q}$ depend on the lowest order neutral pion and eta meson masses in the sea quark sector, which are denoted by $\chi_{\pi}$ and $\chi_{\eta}$. For $d_{\text {sea }}=3$ these are determined by the relations

$$
\begin{aligned}
\chi_{\pi}+\chi_{\eta} & =\frac{2}{3}\left(\chi_{4}+\chi_{5}+\chi_{6}\right), \\
\chi_{\pi} \chi_{\eta} & =\frac{1}{3}\left(\chi_{4} \chi_{5}+\chi_{5} \chi_{6}+\chi_{4} \chi_{6}\right),
\end{aligned}
$$

which have no polynomial solution, but for $d_{\text {sea }}=2$ one has $\chi_{\pi}=\chi_{4}$ and $\chi_{\eta}=1 / 3 \chi_{4}+2 / 3 \chi_{6}$. The propagators for $d_{\text {sea }}=2$ can then be obtained from Eqs. (26) and (27) by taking the appropriate limits. In particular, the terms with a pole in $\chi_{\pi}$ are no longer present. For $d_{\text {sea }}=1$, the fact that $\chi_{\pi}=\chi_{\eta}=\chi_{4}$ gives rise to several further simplifications, and in that case the terms with a pole in $\chi_{\eta}$ vanish as well.

The residues $R$ of the neutral meson propagators in $\mathrm{PQ} \chi \mathrm{PT}$ are in general rational functions of the sea and valence quark masses which can be expressed in terms of the more general quantities $R_{a \ldots b}^{z}$ defined by

$$
\begin{aligned}
R_{a b}^{z} & =\chi_{a}-\chi_{b}, \\
R_{a b c}^{z} & =\frac{\chi_{a}-\chi_{b}}{\chi_{a}-\chi_{c}}, \\
R_{a b c d}^{z} & =\frac{\left(\chi_{a}-\chi_{b}\right)\left(\chi_{a}-\chi_{c}\right)}{\chi_{a}-\chi_{d}}, \\
R_{a b c d e f g}^{z} & =\frac{\left(\chi_{a}-\chi_{b}\right)\left(\chi_{a}-\chi_{c}\right)\left(\chi_{a}-\chi_{d}\right)}{\left(\chi_{a}-\chi_{e}\right)\left(\chi_{a}-\chi_{f}\right)\left(\chi_{a}-\chi_{g}\right)},
\end{aligned}
$$

and so on. Note that $R_{a \ldots b}^{z}$ has the same dimension as $\chi_{i}$ for an even number of indices and is dimensionless for an odd number of indices. For the case of $d_{\text {sea }}=3$, the residues generated by Eqs. (26) and (27) are

$$
\begin{aligned}
R_{j k l}^{i} & =R_{i 456 j k l}^{z}, \\
R_{i}^{d} & =R_{i 456 \pi \eta}^{z}, \\
R_{i}^{c} & =R_{4 \pi \eta}^{i}+R_{5 \pi \eta}^{i}+R_{6 \pi \eta}^{i}-R_{\pi \eta \eta}^{i}-R_{\pi \pi \eta}^{i} .
\end{aligned}
$$

From these definitions, it is apparent that residues of the type $R_{i}^{d}$ or $R_{j \pi \eta}^{i}$ vanish if $i$ is a sea-quark index. Thus the propagators in the sea-quark sector of $\mathrm{PQ} \chi \mathrm{PT}$ contain no double poles as expected, since the origin of the double poles lies in the quenching of the valence quark loops. For the case of $d_{\text {sea }}=2$, due to the cancellations in the seaquark sector (as discussed above) the residues simplify to

$$
\begin{aligned}
R_{j k}^{i} & =R_{i 46 j k}^{z}, \\
R_{i}^{d} & =R_{i 46 \eta}^{z}, \\
R_{i}^{c} & =R_{4 \eta}^{i}+R_{6 \eta}^{i}-R_{\eta \eta}^{i},
\end{aligned}
$$

so that the index $\pi$ no longer appears. For the case of $d_{\text {sea }}=1$, all residues associated with the sea quark sector have reduced to numbers. Some nontrivial residues can still appear if the valence quarks are nondegenerate. These are

$$
\begin{aligned}
R_{j}^{i} & =R_{i 4 j}^{z}, \\
R_{i}^{d} & =R_{i 4}^{z},
\end{aligned}
$$

where the double-pole residue has been retained mainly for notational consistency. Finally, it should be noted that if the sea quark masses are set equal to the valence quark masses, the propagator residues of $\mathrm{PQ} \chi \mathrm{PT}$ reduce so that the $\pi^{0}$ and $\eta$ meson propagators of unquenched $\chi \mathrm{PT}$ are recovered.

Typically, a direct NNLO calculation with the propagators of Eqs. (26) and (27) produces a large number of redundant residues in the output. This problem is especially troublesome for the larger values of $d_{\text {sea }}$ and $d_{\text {val }}$. It is thus useful to note that the various residues $R$ satisfy a large number of algebraic relations, which provide an efficient, albeit tedious, way to simplify and compress the end results of the NNLO calculations. 


\section{Loop Integrals at NNLO}

The expressions for the NNLO masses and decay constants of the charged pseudoscalar mesons in PQ $\chi \mathrm{PT}$ depend on several one- and two-loop integrals. After regularization and renormalization has been carried out, the finite contributions from these integrals are written in terms of the functions $\bar{A}, \bar{B}$ and $\bar{C}$, which are defined as

$$
\begin{aligned}
\bar{A}(\chi) & =-\pi_{16} \chi \log \left(\chi / \mu^{2}\right) \\
\bar{B}\left(\chi_{i}, \chi_{j} ; 0\right) & =-\pi_{16} \frac{\chi_{i} \log \left(\chi_{i} / \mu^{2}\right)-\chi_{j} \log \left(\chi_{j} / \mu^{2}\right)}{\chi_{i}-\chi_{j}} \\
\bar{C}(\chi, \chi, \chi ; 0) & =-\pi_{16} /(2 \chi)
\end{aligned}
$$

where $\mu$ denotes the renormalization scale and $\pi_{16}=$ $1 /\left(16 \pi^{2}\right)$. These integrals are often referred to as chiral logarithms, although the integral $\bar{C}$ does not contain any logarithm when all three arguments are equal. In the often encountered limit $\chi_{i}=\chi_{j}$, the expression for $\bar{B}$ reduces to

$$
\bar{B}(\chi, \chi ; 0)=-\pi_{16}\left(1+\log \left(\chi / \mu^{2}\right)\right) .
$$

The functions described above are in principle sufficient to express all one-loop integrals encountered in the NNLO calculations, but the introduction of further combinations of integrals is desirable in order to reduce the size and complexity of the results. For this purpose, the following three combinations of integrals have been introduced,

$$
\begin{aligned}
\bar{A}(\chi ; \varepsilon) & =\bar{A}(\chi)^{2} /\left(2 \pi_{16} \chi\right) \\
& +\pi_{16} \chi\left(\pi^{2} / 12+1 / 2\right), \\
\bar{B}(\chi, \chi ; 0, \varepsilon) & =\bar{A}(\chi) \bar{B}(\chi, \chi ; 0) /\left(\pi_{16} \chi\right) \\
& -\bar{A}(\chi)^{2} /\left(2 \pi_{16} \chi^{2}\right) \\
& +\pi_{16}\left(\pi^{2} / 12+1 / 2\right), \\
\bar{B}\left(\chi_{i}, \chi_{j} ; 0, k\right) & =\chi_{i} \bar{B}\left(\chi_{i}, \chi_{j} ; 0\right)+\bar{A}\left(\chi_{j}\right),
\end{aligned}
$$

of which the first two expressions are naturally generated by the dimensional regularization procedure, whereas the third one is useful since it is symmetric under the interchange of $\chi_{i}$ and $\chi_{j}$.

The NNLO calculation of pseudoscalar meson masses and decay constants also introduces a number of genuine, nonfactorizable two-loop integrals, which can be evaluated using a generalization of the methods described in Ref. 20]. The two-loop integrals encountered have the following general structure,

$$
\begin{aligned}
\langle\langle X\rangle\rangle= & \frac{1}{i^{2}} \int \frac{d^{d} q}{(2 \pi)^{d}} \frac{d^{d} r}{(2 \pi)^{d}} \times \\
& \frac{X}{\left(q^{2}-\chi_{1}\right)^{a}\left(r^{2}-\chi_{2}\right)^{b}\left((q+r-p)^{2}-\chi_{3}\right)^{c}}
\end{aligned}
$$

where $a, b, c=1$ or 2 , and $X$ represents the different combinations of momentum factors $q$ and $r$ that can occur. The integrals thus generated by Eq. (36) are sometimes referred to as sunset integrals, and they can be expressed in terms of the $H$ functions according to the definitions in Ref. 20]. The $H$ functions satisfy a number of integral relations such that only $H, H_{1}$ and $H_{21}$ are required to express all sunset integrals encountered. Furthermore, after regularization of the $H$ functions, only the finite parts denoted $H^{F}, H_{1}^{F}$ and $H_{21}^{F}$ appear in the end results. In the case of the decay constants, sunset integrals differentiated with respect to $p^{2}$ are also required, and are denoted by the primed quantities $H^{F^{\prime}}, H_{1}^{F^{\prime}}$ and $H_{21}^{F^{\prime}}$.

However, the appearance of double poles in the neutral meson propagators of Eqs. (26) and (27) leads to a significant complication in Eq. (36), as cases with $a, b, c \neq 1$ will then show up. This added layer of complexity is accounted for by an extra integer argument $n$, which indicates the pole configuration of Eq. (36) according to Table II The finite parts of the $H$ functions are thus generalized as

$$
H^{F}\left(\chi_{i}, \chi_{j}, \chi_{k} ; p^{2}\right) \rightarrow H^{F}\left(n, \chi_{i}, \chi_{j}, \chi_{k} ; p^{2}\right),
$$

and similarly for the $H_{1}^{F}$ and $H_{21}^{F}$. In principle, eight different configurations can show up, and the value of $n$ for each one of them is given in Table III However, this expanded set of $H$ functions obeys a generalization of the symmetries (under the exchange of mass arguments) discussed in Ref. [20], and thus some of the pole configurations turn out to be redundant, such that only $n=1,2,3,5$ and 7 are required for a complete NNLO calculation of the pseudoscalar meson masses and decay constants. The case with $n=8$ would however appear for a NNLO calculation of the coefficient of the double pole in the neutral meson propagator. Such a calculation has not yet been performed, but it should be noted that a NLO calculation of that quantity has been published in Ref. 7].

TABLE II: Overview of the notation for the possible configurations of double poles in the $H$ functions generated by Eq. (36) in PQ $\chi \mathrm{PT}$. Redundant configurations are given in parentheses.

\begin{tabular}{c|ccc} 
& $a$ & $b$ & $c$ \\
\hline \hline$n=1$ & 1 & 1 & 1 \\
\hline$n=2$ & 2 & 1 & 1 \\
$n=3$ & 1 & 2 & 1 \\
$(n=4)$ & 1 & 1 & 2 \\
\hline$n=5$ & 2 & 2 & 1 \\
$(n=6)$ & 2 & 1 & 2 \\
$n=7$ & 1 & 2 & 2 \\
\hline$n=8$ & 2 & 2 & 2
\end{tabular}


The explicit expressions for the expanded set of $H$ functions with $n \neq 1$ can be obtained by differentiation with respect to the masses of the expressions for $n=1$ given in Ref. 20]. It should also be noted that the finite contributions from the set of $H$ functions contain integrals which have to be evaluated by numerical integration, and these are again generalizations of the integrals for $n=1$ in Ref. [20].

\section{E. Notation for NNLO Results}

The length of the final NNLO expressions presents a problem. One major culprit has already been identified in the discussion of the $\mathrm{PQ}$ propagator residues, but even after that problem is dealt with, the size of the PQ expressions far exceeds that of the analogous ones in unquenched $\chi \mathrm{PT}$. One reason for this is that the NNLO results in $\mathrm{PQ} \chi \mathrm{PT}$ are highly symmetric under the interchange of quark masses, both in the sea and valence quark sectors, and thus contain a lot of unnecessary repetition. This observation suggests that the NNLO expressions could be efficiently compressed by summation over sea and valence quark indices.

The summation conventions in the sea-quark sector are implemented through two new indices $s$ and $t$, which can appear as indices of explicit quark masses $\chi$, as well as among the indices of the residue functions $R$ or the predefined combinations thereof. These sea-quark summation indices should be interpreted as follows: If an index $t$ is present once or several times, then there will always be an occurrence of the $s$ index as well, and the entire term is then to be summed over all pairs of different sea quark indices. If the index $s$ is present but $t$ is not, then the entire term is to be summed over all sea quark indices. Elementary examples are

$$
\begin{aligned}
\chi_{s} & =\sum_{i=4,5,6} \chi_{i}, \\
\chi_{s t} & =\sum_{(i j)=(45),(46),(56)} \chi_{i j}
\end{aligned}
$$

Thus all the terms in the end result, where the dependence on the sea-quark masses is expressed in terms of the indices $s$ and $t$, explicitly have the (required) symmetry under interchange of sea-quark masses. It should be noted that the summation is over all three sea-quark flavors irrespective of the value of $d_{\text {sea }}$.

Further symmetries exist which can also be used to advantage in the compactification of the NNLO results. Firstly, the valence quark sector has a symmetry under the interchange of valence quark masses. This symmetry has been implemented by introduction of the summation indices $p$ and $q$. In this paper, they are necessary only for the expressions with $d_{\mathrm{val}}=2$, and then always occur for the valence quark masses $\chi_{1}$ and $\chi_{3}$. If the index $q$ is present, there will always be an index $p$ and the resulting sum is over the pairs $(p, q)=(1,3)$ and $(p, q)=(3,1)$. If only $p$ is present, the sum is over the indices 1 and 3 . As an example, consider

$$
\bar{A}\left(\chi_{p}\right) R_{q \eta}^{p} \chi_{p}=\bar{A}\left(\chi_{1}\right) R_{3 \eta}^{1} \chi_{1}+[1 \leftrightarrow 3],
$$

which demonstrates that any contribution written in terms of the $(p, q)$ notation is symmetric (as required) under the interchange of the valence quark masses $\chi_{1}$ and $\chi_{3}$. Secondly, for $d_{\text {sea }}=3$ the sea-quark sector exhibits an additional symmetry, under the interchange of the lowest order neutral meson masses $\chi_{\pi}$ and $\chi_{\eta}$. This symmetry has been implemented by the indices $m$ and $n$. If the index $m$ is present, there will always be an index $n$ and the corresponding sum is over the pairs $(m, n)=(\pi, \eta)$ and $(m, n)=(\eta, \pi)$. For example

$$
\bar{A}\left(\chi_{m}\right) R_{n 11}^{m} \chi_{m}=\bar{A}\left(\chi_{\eta}\right) R_{\pi 11}^{\eta} \chi_{\eta}+[\eta \leftrightarrow \pi] .
$$

Similar to the earlier cases, if only the index $n$ is present, then the term is to be summed over the $\chi_{\pi}$ and $\chi_{\eta}$ masses.

The summation techniques described above already incorporate most of the recurring combinations of terms in the end results, which has the added benefit of avoiding the introduction of an unreasonable amount of specialized notation. However, certain combinations of quark masses $\chi$ and propagator residues $R$ appear throughout the results and have therefore been given dedicated names. As an example, combinations of the type

$$
\begin{aligned}
\bar{\chi}_{g} & =\frac{1}{3} \sum_{i=4,5,6} \chi_{i}^{g}, \\
\bar{\chi}_{b g}^{a} & =\frac{1}{3} \sum_{i=4,5,6} R_{b i i}^{a} \chi_{i}^{g},
\end{aligned}
$$

where the index $g$ indicates the power of the sea-quark masses averaged, appear throughout the expressions. The corresponding ones for $d_{\text {sea }}=2$ can be obtained by setting $R_{b i i}^{a} \rightarrow R_{b i}^{a}$. Again, it should be kept in mind that the summation always runs over all three quark flavors, regardless of the value of $d_{\text {sea }}$. The calculation of the pseudoscalar meson decay constant of Ref. [10] made use of many more complicated averages of the above type, with products of up to three residues. However, most of the quantities $\bar{\chi}$ which involve the residues $R$ can be reexpressed in terms of simpler quantities, such that e.g. $\bar{\chi}_{\eta 0}^{\pi}=-1, \bar{\chi}_{\eta 1}^{\pi}=-\chi_{\pi}$ and $\bar{\chi}_{\pi 1}^{\eta}=-\chi_{\eta}$. The use of the $\bar{\chi}$ notation has therefore been discontinued, and the only quantities of the type given in Eq. (41) that are used in this paper are $\bar{\chi}_{1}$ and $\bar{\chi}_{2}$. The other named combinations consist of sums of products of quark masses and propagator residues. For $d_{\text {sea }}=3$, these are

$$
\begin{aligned}
R_{i j k l}^{v} & =R_{j k k}^{i}+R_{j l l}^{i}-2 R_{j k l}^{i}, \\
R_{i j k l}^{u} & =R_{\pi \eta k}^{i} R_{\pi \eta k}^{j}-R_{\pi \eta k}^{i} R_{\pi \eta l}^{j} \\
& -R_{\pi \eta l}^{i} R_{\pi \eta k}^{j}+R_{\pi \eta l}^{i} R_{\pi \eta l}^{j}, \\
R_{i j k l}^{u m} & =R_{m m^{\prime} k}^{i} R_{m^{\prime} j k}^{m}-R_{m m^{\prime} k}^{i} R_{m^{\prime} j l}^{m} \\
& -R_{m m^{\prime} l}^{i} R_{m^{\prime} j k}^{m}+R_{m m^{\prime} l}^{i} R_{m^{\prime} j l}^{m},
\end{aligned}
$$




$$
\begin{aligned}
R_{i j k l}^{u m n} & =R_{m^{\prime} i k}^{m} R_{n^{\prime} j k}^{n}-R_{m^{\prime} i k}^{m} R_{n^{\prime} j l}^{n} \\
& -R_{m^{\prime} i l}^{m} R_{n^{\prime} j k}^{n}+R_{m^{\prime} i l}^{m} R_{n^{\prime} j l}^{n}, \\
R_{i j k l}^{w} & =R_{\pi \eta k}^{i} R_{\pi \eta k}^{j} \chi_{k}+2 R_{\pi \eta k}^{i} R_{\pi \eta l}^{j} \chi_{k l} \\
& +2 R_{\pi \eta l}^{i} R_{\pi \eta k}^{j} \chi_{k l}+R_{\pi \eta l}^{i} R_{\pi \eta l}^{j} \chi_{l}, \\
R_{i j k l}^{w m} & =R_{m m^{\prime} k}^{i} R_{m^{\prime} j k}^{m} \chi_{k}+2 R_{m m^{\prime} k}^{i} R_{m^{\prime} j l}^{m} \chi_{k l} \\
& +2 R_{m m^{\prime} l}^{i} R_{m^{\prime} j k}^{m} \chi_{k l}+R_{m m^{\prime} l}^{i} R_{m^{\prime} j l}^{m} \chi_{l}, \\
R_{i j k l}^{w m n} & =R_{m^{\prime} i k}^{m} R_{n^{\prime} j k}^{n} \chi_{k}+2 R_{m^{\prime} i k}^{m} R_{n^{\prime} j l}^{n} \chi_{k l} \\
& +2 R_{m^{\prime} i l}^{m} R_{n^{\prime} j k}^{n} \chi_{k l}+R_{m^{\prime} i l}^{m} R_{n^{\prime} j l}^{n} \chi_{l},
\end{aligned}
$$

and the analogous ones for $d_{\text {sea }}=2$ are

$$
\begin{aligned}
R_{i j k}^{v} & =R_{j j}^{i}+R_{k k}^{i}-2 R_{j k}^{i}, \\
R_{i j k l}^{u} & =R_{\eta k}^{i} R_{\eta k}^{j}-R_{\eta k}^{i} R_{\eta l}^{j} \\
& -R_{\eta l}^{i} R_{\eta k}^{j}+R_{\eta l}^{i} R_{\eta l}^{j}, \\
R_{i j k l}^{u m} & =R_{m k}^{i} R_{j k}^{m}-R_{m k}^{i} R_{j l}^{m} \\
& -R_{m l}^{i} R_{j k}^{m}+R_{m l}^{i} R_{j l}^{m}, \\
R_{i j k l}^{w} & =R_{\eta k}^{i} R_{\eta k}^{j} \chi_{k}+2 R_{\eta k}^{i} R_{\eta l}^{j} \chi_{k l} \\
& +2 R_{\eta l}^{i} R_{\eta k}^{j} \chi_{k l}+R_{\eta l}^{i} R_{\eta l}^{j} \chi_{l}, \\
R_{i j k l}^{w m} & =R_{m k}^{i} R_{j k}^{m} \chi_{k}+2 R_{m k}^{i} R_{j l}^{m} \chi_{k l} \\
& +2 R_{m l}^{i} R_{j k}^{m} \chi_{k l}+R_{m l}^{i} R_{j l}^{m} \chi_{l} .
\end{aligned}
$$

For the $R^{u}$ and $R^{w}$ type terms, the indices $m, n$ denote either $\pi$ or $\eta$ (independently of each other). If $m$ denotes a $\pi$ then the corresponding index $m^{\prime}$ denotes an $\eta$ and vice versa. Similarly an $n$ denoting a $\pi$ implies that the $n^{\prime}$ denotes an $\eta$. As an example, consider

$$
\begin{aligned}
R_{i j k l}^{u \pi \eta} & =R_{\eta i k}^{\pi} R_{\pi j k}^{\eta}-R_{\eta i k}^{\pi} R_{\pi j l}^{\eta} \\
& -R_{\eta i l}^{\pi} R_{\pi j k}^{\eta}+R_{\eta i l}^{\pi} R_{\pi j l}^{\eta},
\end{aligned}
$$

which should suffice to explain the usage of the indices $m, n$ and $m^{\prime}, n^{\prime}$. Note that this particular combination is symmetric under the interchange of $\pi$ and $\eta$ only if $i=j$, which means that this particular $R^{u}$ can appear in the final result only in combinations that are symmetric in the indices $i$ and $j$.

For the case of $d_{\text {sea }}=2$, the indices $m^{\prime}, n^{\prime}$ no longer appear, since $\chi_{\pi}=\chi_{4}$ and has therefore canceled out from the expressions. In fact, the index $m$ in Eq. (43) always represents an $\eta$. However, Eq. (43) has been written in a slightly more formal way to illustrate the similarity with the expressions for $d_{\text {sea }}=3$. It is actually possible to obtain the expressions for $d_{\text {sea }}=2$ from the corresponding ones for $d_{\text {sea }}=3$ in Eq. (42) by removing all occurrences of the indices $\pi$ and $m^{\prime}$ there. It should be noted that the letters $u, v, w$ in $R^{u}, R^{v}$ and $R^{w}$ are not indices, but rather indicate different types of residue combinations. The indices $m, n$ used in the above formulas have nothing to do with the summation indices $m, n$ defined earlier, which are encountered in the analytical expressions in the next sections. In Eqs. (42) and (43) they only indicate an occurrence of either a $\pi$ or an $\eta$.
The implementation of the flavor permutation symmetries described in this section is a complicated task, as the direct output of a NNLO calculation of masses or decay constants using the propagators defined in Eqs. (26) and (27) produces highly redundant expressions. Especially for the higher values of $d_{\text {sea }}$ and $d_{\text {val }}$, the direct output sometimes consists of tens of thousands of terms. Thus the output has to be cleaned up using the many algebraic relations between the residues $R$, before any attempt at implementing the flavor permutation symmetries can be made. This process involves the factorization of up to $\sim 200$ different expressions of varying length and complexity. As an example for $d_{\text {val }}=2$, the parts of the direct output proportional to $\bar{A}\left(\chi_{\eta} ; \varepsilon\right)$ and $\bar{A}\left(\chi_{1} ; \varepsilon\right)$ alone are hundreds of terms long, while in factorized and simplified form, they contain only $\sim 5$ terms. After considerable trial and error in Maple, the simplification of the results was successfully carried through. In addition, the final simplified results have been checked algebraically against the original (long) ones.

\section{ANALYTICAL RESULTS FOR THE MASSES}

\section{A. Masses at NNLO}

The corrections to the mass of a pseudoscalar meson are obtained by consideration of the self-energy contributions to the propagator of the interacting field theory. That propagator is defined in terms of the Fourier transform of the two-point Green's function,

$$
i \Delta(p)=\int d^{4} x e^{i p \cdot x}\left\langle\Omega\left|T\left[\Phi(x)_{j i} \Phi(0)_{i j}\right]\right| \Omega\right\rangle,
$$

where $\Phi_{i j}=q_{i} \bar{q}_{j}$ denotes any of the off-diagonal mesons in the valence sector of $\mathrm{PQ} \chi \mathrm{PT}$, and $\Omega$ denotes the vacuum of the interacting theory. To lowest order, $i \Delta(p)=$ $G_{i j}^{c}(p)$ of Eq. 231). When written in terms of the fields in the Lagrangian, the resulting self-energy diagrams can be summed as a geometric series [21], giving

$$
i \Delta(p)=\frac{i}{p^{2}-M_{0}^{2}-\Sigma\left(p^{2}, \chi_{i}\right)},
$$

where $M_{0}^{2}$ denotes the lowest order mass of the meson which is being considered, and $\chi_{i}$ in $\Sigma$ denotes the dependence of the self-energy on all the lowest order meson masses. The quantity $\Sigma\left(p^{2}, \chi_{i}\right)$ takes into account the contributions from the one-particle-irreducible (1PI) diagrams. The physical masses, which include the interaction, of the off-diagonal mesons in $\mathrm{PQ} \chi \mathrm{PT}$ are defined by the position of the pole in Eq. [46],

$$
M_{\text {phys }}^{2}=M_{0}^{2}+\Sigma\left(M_{\text {phys }}^{2}, \chi_{i}\right),
$$

whereas the propagators of the neutral or diagonal

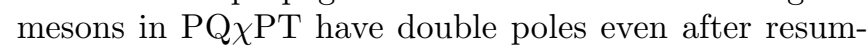
mation. 


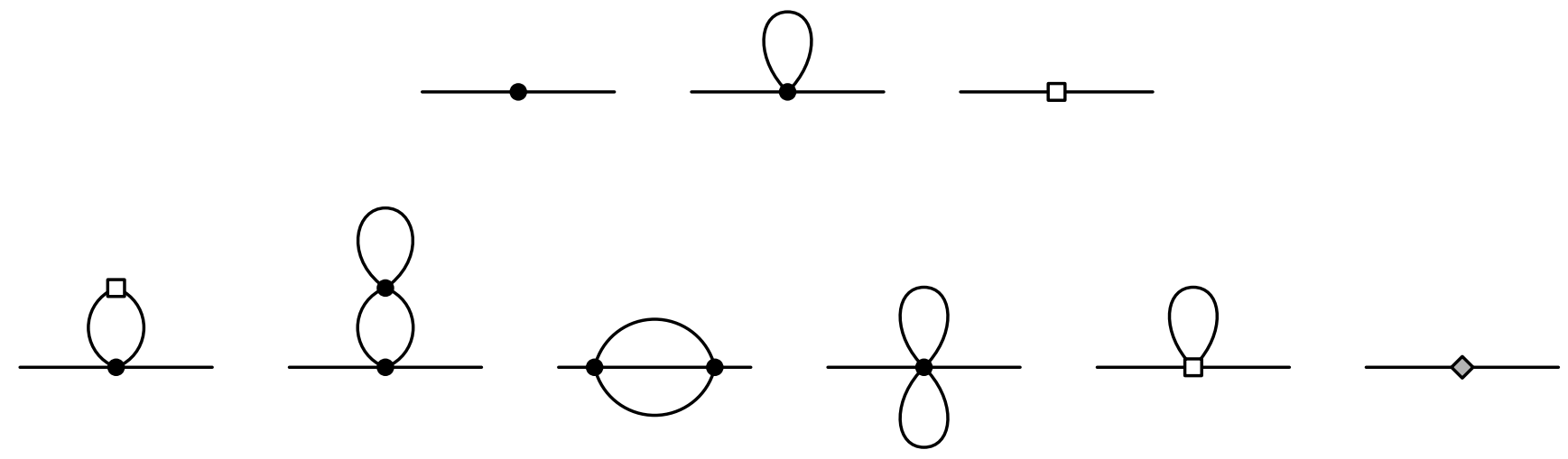

FIG. 1: Feynman diagrams up to $\mathcal{O}\left(p^{6}\right)$ or two loops, for the self-energy $\Sigma\left(M_{\text {phys }}^{2}, \chi_{i}\right)$. Filled circles denote vertices of the $\mathcal{L}_{2}$ Lagrangian, whereas open squares and diamonds denote vertices of the $\mathcal{L}_{4}$ and $\mathcal{L}_{6}$ Lagrangians, respectively. In the top row, the first diagram from the left is of $\mathcal{O}\left(p^{2}\right)$, whereas the other two diagrams are of $\mathcal{O}\left(p^{4}\right)$. The diagrams of $\mathcal{O}\left(p^{6}\right)$, which give the NNLO correction to the meson mass, are shown in the bottom row. Of those, the third diagram from the left is called the "sunset" diagram in the text.

The expression for the self-energy $\Sigma$ can be written as a string of terms which denote the 1PI diagrams of progressively higher order,

$$
\begin{aligned}
\Sigma\left(M_{\text {phys }}^{2}, \chi_{i}\right) & =\Sigma_{4}\left(M_{\text {phys }}^{2}, \chi_{i}\right) \\
& +\Sigma_{6}\left(M_{\text {phys }}^{2}, \chi_{i}\right)+\mathcal{O}\left(p^{8}\right),
\end{aligned}
$$

where $\Sigma_{4}$ contains the self-energy diagrams of $\mathcal{O}\left(p^{4}\right)$, and $\Sigma_{6}$ those of $\mathcal{O}\left(p^{6}\right)$. For the present NNLO calculation, the form of $M_{\text {phys }}^{2}$ has to be determined up to $\mathcal{O}\left(p^{6}\right)$. It is sufficient, for this purpose, to use the lowest order mass instead of $M_{\text {phys }}^{2}$ in $\Sigma_{6}$ since the diagrams in that term are already of $\mathcal{O}\left(p^{6}\right)$. However, in the case of $\Sigma_{4}$ the argument $M_{\text {phys }}^{2}$ has to be expanded in a Taylor series around $M_{0}^{2}$, since the diagrams in $\Sigma_{4}$ are of $\mathcal{O}\left(p^{4}\right)$. If $M_{\text {phys }}^{2}$ is formally written as

$$
M_{\text {phys }}^{2}=M_{0}^{2}+M_{4}^{2}+M_{6}^{2}+\mathcal{O}\left(p^{8}\right),
$$

then an expansion of $\Sigma_{4}$ up to $\mathcal{O}\left(p^{6}\right)$ gives

$$
\begin{aligned}
\Sigma_{4}\left(M_{\text {phys }}^{2}, \chi_{i}\right) & =\Sigma_{4}\left(M_{0}^{2}, \chi_{i}\right)+\left.M_{4}^{2} \frac{\partial \Sigma_{4}\left(p^{2}, \chi_{i}\right)}{\partial p^{2}}\right|_{M_{0}^{2}} \\
& +\mathcal{O}\left(p^{8}\right),
\end{aligned}
$$

where $M_{4}^{2}$ represents the NLO correction to the lowest order meson mass, which is given by $\Sigma_{4}\left(M_{0}^{2}, \chi_{i}\right)$. The final formula for the pseudoscalar meson mass to $\mathcal{O}\left(p^{6}\right)$ is thus

$$
\begin{aligned}
M_{\text {phys }}^{2} & =M_{0}^{2}+\Sigma_{4}\left(M_{0}^{2}, \chi_{i}\right) \\
& +\left.\Sigma_{4}\left(M_{0}^{2}, \chi_{i}\right) \frac{\partial \Sigma_{4}\left(p^{2}, \chi_{i}\right)}{\partial p^{2}}\right|_{M_{0}^{2}} \\
& +\Sigma_{6}\left(M_{0}^{2}, \chi_{i}\right)+\mathcal{O}\left(p^{8}\right),
\end{aligned}
$$

where the last two terms are of $\mathcal{O}\left(p^{6}\right)$ and represent the NNLO correction to the lowest order mass. The Feynman diagrams that contribute to $\Sigma_{6}\left(M_{0}^{2}, \chi_{i}\right)$ are shown in Fig. 11 In the case of the self energies at NNLO, the $\mathcal{O}\left(p^{6}\right)$ vertex introduces the LEC:s $K_{17}^{r}$ through $K_{27}^{r}, K_{39}^{r}$ and $K_{40}^{r}$.

The physical mass of a pseudoscalar meson $\Phi_{i j}$ is given to NNLO in the form

$$
M_{\mathrm{phys}}^{2}=\chi_{i j}+\frac{\delta^{(4) \mathrm{vs}}}{F_{0}^{2}}+\frac{\delta_{\mathrm{ct}}^{(6) \mathrm{vs}}+\delta_{\text {loops }}^{(6) \mathrm{vs}}}{F_{0}^{4}}+\mathcal{O}\left(p^{8}\right),
$$

where the LO result $M_{0}^{2}=\chi_{i j}$ has already been inserted, and the $\mathcal{O}\left(p^{4}\right)$ and $\mathcal{O}\left(p^{6}\right)$ contributions separated. The NNLO contribution $\delta^{(6)}$ has been further split into the contributions from the chiral loops and from the $\mathcal{O}\left(p^{6}\right)$ counterterms or LEC:s. The superscripts (v) and (s) indicate the values of $d_{\mathrm{val}}$ and $d_{\mathrm{sea}}$, respectively. It should be noted that we have chosen to give the results to the various orders in terms of the lowest order decay constant $F_{0}$ and in terms of the lowest order masses, since these are the fundamental inputs in $\mathrm{PQ} \chi \mathrm{PT}$. The situation is different in standard $\chi \mathrm{PT}$, where the main objective is comparison with experiment, in which case the formulas are most often rewritten in terms of the physical decay constants and masses.

\section{B. Expressions for $d_{\mathrm{val}}=1$}

In the previously published paper on the NNLO meson masses in PQ $\chi \mathrm{PT}[9]$, which treated only the most degenerate $(1+1)$ mass case, an overall factor $\chi_{1}$ was factored out from the analytical expressions. However, it has turned out that it is not possible to find such an 
overall factor in a meaningful way for the less degenerate mass cases. This is especially evident for $d_{\text {val }}=2$. Thus, for consistency, the factor $\chi_{1}$ has not been factored out from the expression for the $(1+1)$ mass case reproduced here either. That expression also uses the new $R$ notation as well as a more efficient set of loop integrals, but it is of course equivalent to the one presented previously in Ref. 9].

The NLO result for $d_{\text {val }}=1$ is rather short, and therefore it suffices to give this expression for $d_{\text {sea }}=3$ only. The corresponding results for $d_{\text {sea }}=1,2$ can easily be derived from that expression by consideration of the appropriate limits, i.e. $\chi_{5} \rightarrow \chi_{4}$ for $d_{\text {sea }}=2$ and $\chi_{5}, \chi_{6} \rightarrow \chi_{4}$ for $d_{\text {sea }}=1$. The combined NLO result (loops and counterterms), is

$$
\begin{aligned}
\delta^{(4) 13}= & -24 L_{4}^{r} \bar{\chi}_{1} \chi_{1}-8 L_{5}^{r} \chi_{1}^{2}+48 L_{6}^{r} \bar{\chi}_{1} \chi_{1} \\
& +16 L_{8}^{r} \chi_{1}^{2}-1 / 3 \bar{A}\left(\chi_{m}\right) R_{n 11}^{m} \chi_{1} \\
& -1 / 3 \bar{A}\left(\chi_{1}\right) R_{1}^{c} \chi_{1} \\
& -1 / 3 \bar{B}\left(\chi_{1}, \chi_{1}, 0\right) R_{1}^{d} \chi_{1}
\end{aligned}
$$

which is in agreement with Refs. 3, 7]. The NNLO result has been split into the contribution from the loop diagrams and the contribution from the $\mathcal{O}\left(p^{6}\right)$ counterterms. The latter consists of the finite part of the rightmost diagram in Fig. 11 and is given for $d_{\text {sea }}=3$ by

$$
\begin{aligned}
\delta_{\mathrm{ct}}^{(6) 13}= & -32 K_{17}^{r} \chi_{1}^{3}-96 K_{18}^{r} \bar{\chi}_{1} \chi_{1}^{2}-16 K_{19}^{r} \chi_{1}^{3} \\
& -48 K_{20}^{r} \bar{\chi}_{1} \chi_{1}^{2}-48 K_{21}^{r} \bar{\chi}_{2} \chi_{1} \\
& -144 K_{22}^{r} \bar{\chi}_{1}^{2} \chi_{1}-16 K_{23}^{r} \chi_{1}^{3}+48 K_{25}^{r} \chi_{1}^{3} \\
& +K_{26}^{r}\left[96 \bar{\chi}_{1} \chi_{1}^{2}+48 \bar{\chi}_{2} \chi_{1}\right]+432 K_{27}^{r} \bar{\chi}_{1}^{2} \chi_{1} \\
& +32 K_{39}^{r} \chi_{1}^{3}+96 K_{40}^{r} \bar{\chi}_{1} \chi_{1}^{2} .
\end{aligned}
$$

As for the NLO result above, the contributions from the $\mathcal{O}\left(p^{6}\right)$ counterterms for $d_{\text {sea }}=1,2$ can be derived straightforwardly by taking the appropriate mass limits of this expression.

However, the situation is different for the loop contribution at NNLO, since it is much larger and has a rather complicated structure. This makes it a difficult task to derive the results for $d_{\text {sea }}=1,2$ directly from the $d_{\text {sea }}=3$ case, and therefore the different cases are given separately below. The need for such expressions is clear since many Lattice QCD simulations work with $d_{\text {sea }}=2$ rather than $d_{\text {sea }}=3$. As expected, all infinities have canceled for all expressions in the renormalization procedure, and the result for $\delta_{\text {loops }}^{(6) 11}$ is equivalent to the one published in Ref. [9]. The FORM [22] output with these expressions can be downloaded from the website 14]. The chiral loop contributions to the pseudoscalar meson mass at $\mathrm{NNLO}$ in $\mathrm{PQ} \chi \mathrm{PT}$, for $d_{\mathrm{val}}=1$, are

$$
\begin{aligned}
& \delta_{\text {loops }}^{(6) 11}=\pi_{16} L_{0}^{r}\left[3 \chi_{1} \chi_{4}^{2}+26 / 3 \chi_{1}^{2} \chi_{4}-\chi_{1}^{3}\right]+4 \pi_{16} L_{1}^{r} \chi_{1}^{3}+\pi_{16} L_{2}^{r}\left[16 \chi_{1} \chi_{4}^{2}+2 \chi_{1}^{3}\right]+\pi_{16} L_{3}^{r}\left[3 / 2 \chi_{1} \chi_{4}^{2}\right. \\
& \left.+17 / 3 \chi_{1}^{2} \chi_{4}-5 / 2 \chi_{1}^{3}\right]+\pi_{16}^{2}\left[73 / 64 \chi_{1} \chi_{4}^{2}+15 / 32 \chi_{1}^{2} \chi_{4}-3 / 32 \chi_{1}^{3}\right]+384 L_{4}^{r} L_{5}^{r} \chi_{1}^{2} \chi_{4}-1152 L_{4}^{r} L_{6}^{r} \chi_{1} \chi_{4}^{2} \\
& -384 L_{4}^{r} L_{8}^{r} \chi_{1}^{2} \chi_{4}+576 L_{4}^{r 2} \chi_{1} \chi_{4}^{2}-384 L_{5}^{r} L_{6}^{r} \chi_{1}^{2} \chi_{4}-128 L_{5}^{r} L_{8}^{r} \chi_{1}^{3}+64 L_{5}^{r 2} \chi_{1}^{3}-8 \bar{A}\left(\chi_{1}\right) L_{0}^{r}\left[\chi_{1}^{2}\right. \\
& \left.+R_{1}^{d} \chi_{1}\right]+8 \bar{A}\left(\chi_{1}\right) L_{1}^{r} \chi_{1}^{2}+20 \bar{A}\left(\chi_{1}\right) L_{2}^{r} \chi_{1}^{2}-8 \bar{A}\left(\chi_{1}\right) L_{3}^{r}\left[\chi_{1}^{2}+R_{1}^{d} \chi_{1}\right]+16 \bar{A}\left(\chi_{1}\right) L_{4}^{r} \chi_{1} \chi_{4} \\
& +\bar{A}\left(\chi_{1}\right) L_{5}^{r}\left[32 / 3 \chi_{1}^{2}+16 / 3 R_{1}^{d} \chi_{1}\right]-\bar{A}\left(\chi_{1}\right) L_{6}^{r}\left[16 \chi_{1} \chi_{4}-32 \chi_{1}^{2}\right]+32 \bar{A}\left(\chi_{1}\right) L_{7}^{r} R_{1}^{d} \chi_{1} \\
& \text { - } 64 / 3 \bar{A}\left(\chi_{1}\right) L_{8}^{r} \chi_{1}^{2}+5 / 9 \bar{A}\left(\chi_{1}\right)^{2} \chi_{1}+\bar{A}\left(\chi_{1}\right) \bar{B}\left(\chi_{1}, \chi_{1}, 0\right)\left[11 / 9 \chi_{1}^{2}+1 / 9 R_{1}^{d} \chi_{1}\right] \\
& +2 / 9 \bar{A}\left(\chi_{1}\right) \bar{C}\left(\chi_{1}, \chi_{1}, \chi_{1}, 0\right) R_{1}^{d} \chi_{1}^{2}-\bar{A}\left(\chi_{1}, \varepsilon\right) \pi_{16}\left[11 / 12 \chi_{1}^{2}-1 / 4 R_{1}^{d} \chi_{1}\right]+3 \bar{A}\left(\chi_{14}\right) \pi_{16} \chi_{1} \chi_{4} \\
& +24 \bar{A}\left(\chi_{14}\right) L_{0}^{r} \chi_{1} \chi_{14}+60 \bar{A}\left(\chi_{14}\right) L_{3}^{r} \chi_{1} \chi_{14}-48 \bar{A}\left(\chi_{14}\right) L_{5}^{r} \chi_{1} \chi_{14}+96 \bar{A}\left(\chi_{14}\right) L_{8}^{r} \chi_{1} \chi_{14}-9 / 4 \bar{A}\left(\chi_{14}\right)^{2} \chi_{1} \\
& -2 \bar{A}\left(\chi_{14}\right) \bar{B}\left(\chi_{1}, \chi_{1}, 0\right) \chi_{1} \chi_{4}-\bar{A}\left(\chi_{14}, \varepsilon\right) \pi_{16}\left[9 / 2 \chi_{1} \chi_{4}+5 / 2 \chi_{1}^{2}\right]+128 \bar{A}\left(\chi_{4}\right) L_{1}^{r} \chi_{1} \chi_{4} \\
& +32 \bar{A}\left(\chi_{4}\right) L_{2}^{r} \chi_{1} \chi_{4}-128 \bar{A}\left(\chi_{4}\right) L_{4}^{r} \chi_{1} \chi_{4}+128 \bar{A}\left(\chi_{4}\right) L_{6}^{r} \chi_{1} \chi_{4}+8 / 9 \bar{A}\left(\chi_{4}\right) \bar{B}\left(\chi_{1}, \chi_{1}, 0\right) \chi_{1} \chi_{4} \\
& -2 \bar{A}\left(\chi_{4}, \varepsilon\right) \pi_{16} \chi_{1} \chi_{4}-8 \bar{B}\left(\chi_{1}, \chi_{1}, 0\right) L_{0}^{r} R_{1}^{d} \chi_{1}^{2}-8 \bar{B}\left(\chi_{1}, \chi_{1}, 0\right) L_{3}^{r} R_{1}^{d} \chi_{1}^{2}+\bar{B}\left(\chi_{1}, \chi_{1}, 0\right) L_{4}^{r}\left[8 \chi_{1}^{2} \chi_{4}\right. \\
& \left.+24 R_{1}^{d} \chi_{1} \chi_{4}\right]+\bar{B}\left(\chi_{1}, \chi_{1}, 0\right) L_{5}^{r}\left[8 / 3 \chi_{1}^{3}+16 R_{1}^{d} \chi_{1}^{2}\right]-\bar{B}\left(\chi_{1}, \chi_{1}, 0\right) L_{6}^{r}\left[16 \chi_{1}^{2} \chi_{4}+32 R_{1}^{d} \chi_{1} \chi_{4}\right] \\
& +16 \bar{B}\left(\chi_{1}, \chi_{1}, 0\right) L_{7}^{r}\left(R_{1}^{d}\right)^{2} \chi_{1}-\bar{B}\left(\chi_{1}, \chi_{1}, 0\right) L_{8}^{r}\left[16 / 3 \chi_{1}^{3}+32 R_{1}^{d} \chi_{1}^{2}-16 / 3\left(R_{1}^{d}\right)^{2} \chi_{1}\right] \\
& +\bar{B}\left(\chi_{1}, \chi_{1}, 0\right)^{2}\left[2 / 9 R_{1}^{d} \chi_{1}^{2}+1 / 18\left(R_{1}^{d}\right)^{2} \chi_{1}\right]+2 / 9 \bar{B}\left(\chi_{1}, \chi_{1}, 0\right) \bar{C}\left(\chi_{1}, \chi_{1}, \chi_{1}, 0\right)\left(R_{1}^{d}\right)^{2} \chi_{1}^{2} \\
& +29 / 36 \bar{B}\left(\chi_{1}, \chi_{1}, 0, \varepsilon\right) \pi_{16} R_{1}^{d} \chi_{1}^{2}+16 \bar{C}\left(\chi_{1}, \chi_{1}, \chi_{1}, 0\right) L_{4}^{r} R_{1}^{d} \chi_{1}^{2} \chi_{4}+16 / 3 \bar{C}\left(\chi_{1}, \chi_{1}, \chi_{1}, 0\right) L_{5}^{r} R_{1}^{d} \chi_{1}^{3} \\
& -32 \bar{C}\left(\chi_{1}, \chi_{1}, \chi_{1}, 0\right) L_{6}^{r} R_{1}^{d} \chi_{1}^{2} \chi_{4}-32 / 3 \bar{C}\left(\chi_{1}, \chi_{1}, \chi_{1}, 0\right) L_{8}^{r} R_{1}^{d} \chi_{1}^{3}+5 / 9 H^{F}\left(1, \chi_{1}, \chi_{1}, \chi_{1}, \chi_{1}\right) \chi_{1}^{2} \\
& +H^{F}\left(1, \chi_{1}, \chi_{14}, \chi_{14}, \chi_{1}\right)\left[1 / 4 \chi_{1} \chi_{4}-\chi_{1}^{2}\right]+2 H^{F}\left(1, \chi_{14}, \chi_{14}, \chi_{4}, \chi_{1}\right) \chi_{1} \chi_{4} \\
& +4 / 9 H^{F}\left(2, \chi_{1}, \chi_{1}, \chi_{1}, \chi_{1}\right) R_{1}^{d} \chi_{1}^{2}+3 / 4 H^{F}\left(2, \chi_{1}, \chi_{14}, \chi_{14}, \chi_{1}\right) R_{1}^{d} \chi_{1}^{2}+2 / 9 H^{F}\left(5, \chi_{1}, \chi_{1}, \chi_{1}, \chi_{1}\right)\left(R_{1}^{d}\right)^{2} \chi_{1}^{2} \\
& -4 H_{1}^{F}\left(3, \chi_{14}, \chi_{1}, \chi_{14}, \chi_{1}\right) R_{1}^{d} \chi_{1}^{2}+3 / 4 H_{21}^{F}\left(1, \chi_{1}, \chi_{14}, \chi_{14}, \chi_{1}\right) \chi_{1}^{2}+6 H_{21}^{F}\left(1, \chi_{4}, \chi_{14}, \chi_{14}, \chi_{1}\right) \chi_{1}^{2} \\
& -3 / 4 H_{21}^{F}\left(2, \chi_{1}, \chi_{14}, \chi_{14}, \chi_{1}\right) R_{1}^{d} \chi_{1}^{2} \text {, }
\end{aligned}
$$




$$
\begin{aligned}
& \delta_{\text {loops }}^{(6) 12}=\pi_{16} L_{0}^{r}\left[-8 / 9 \chi_{\eta} \chi_{1} \chi_{4}-\chi_{1}^{3}+26 / 3 \bar{\chi}_{1} \chi_{1}^{2}+35 / 9 \bar{\chi}_{2} \chi_{1}\right]+4 \pi_{16} L_{1}^{r} \chi_{1}^{3}+\pi_{16} L_{2}^{r}\left[22 / 3 \chi_{\eta} \chi_{1} \chi_{4}+2 \chi_{1}^{3}\right. \\
& \left.+26 / 3 \bar{\chi}_{2} \chi_{1}\right]+\pi_{16} L_{3}^{r}\left[-8 / 9 \chi_{\eta} \chi_{1} \chi_{4}-5 / 2 \chi_{1}^{3}+17 / 3 \bar{\chi}_{1} \chi_{1}^{2}+43 / 18 \bar{\chi}_{2} \chi_{1}\right]+\pi_{16}^{2}\left[15 / 32 \chi_{\eta} \chi_{1} \chi_{4}\right. \\
& \left.-3 / 32 \chi_{1}^{3}+15 / 32 \bar{\chi}_{1} \chi_{1}^{2}+43 / 64 \bar{\chi}_{2} \chi_{1}\right]+384 L_{4}^{r} L_{5}^{r} \bar{\chi}_{1} \chi_{1}^{2}-1152 L_{4}^{r} L_{6}^{r} \bar{\chi}_{1}^{2} \chi_{1}-384 L_{4}^{r} L_{8}^{r} \bar{\chi}_{1} \chi_{1}^{2} \\
& +576 L_{4}^{r 2} \bar{\chi}_{1}^{2} \chi_{1}-384 L_{5}^{r} L_{6}^{r} \bar{\chi}_{1} \chi_{1}^{2}-128 L_{5}^{r} L_{8}^{r} \chi_{1}^{3}+64 L_{5}^{r 2} \chi_{1}^{3}-8 \bar{A}\left(\chi_{\eta}\right) L_{0}^{r} R_{11}^{\eta} \chi_{\eta} \chi_{1} \\
& +16 \bar{A}\left(\chi_{\eta}\right) L_{1}^{r} \chi_{\eta} \chi_{1}+4 \bar{A}\left(\chi_{\eta}\right) L_{2}^{r} \chi_{\eta} \chi_{1}-8 \bar{A}\left(\chi_{\eta}\right) L_{3}^{r} R_{11}^{\eta} \chi_{\eta} \chi_{1}-16 \bar{A}\left(\chi_{\eta}\right) L_{4}^{r}\left[\chi_{\eta} \chi_{1}-\bar{\chi}_{1} R_{11}^{\eta} \chi_{1}\right] \\
& +16 / 3 \bar{A}\left(\chi_{\eta}\right) L_{5}^{r}\left[R_{11}^{\eta} \chi_{\eta} \chi_{1}+R_{11}^{\eta} \chi_{1}^{2}\right]+16 \bar{A}\left(\chi_{\eta}\right) L_{6}^{r}\left[\chi_{\eta} \chi_{1}-\bar{\chi}_{1} R_{11}^{\eta} \chi_{1}\right]+32 \bar{A}\left(\chi_{\eta}\right) L_{7}^{r} R_{\eta 461}^{z} \chi_{1} \\
& \text { - 64/3 } \bar{A}\left(\chi_{\eta}\right) L_{8}^{r} R_{11}^{\eta} \chi_{1}^{2}+1 / 18 \bar{A}\left(\chi_{\eta}\right)^{2}\left(R_{11}^{\eta}\right)^{2} \chi_{1}+1 / 9 \bar{A}\left(\chi_{\eta}\right) \bar{A}\left(\chi_{1}\right) R_{11}^{\eta} R_{1}^{c} \chi_{1}-1 / 3 \bar{A}\left(\chi_{\eta}\right) \bar{A}\left(\chi_{46}\right) R_{11}^{\eta} \chi_{1} \\
& -4 / 9 \bar{A}\left(\chi_{\eta}\right) \bar{A}\left(\chi_{1 s}\right) R_{1 s}^{\eta} R_{1 s \eta}^{z} \chi_{1}+\bar{A}\left(\chi_{\eta}\right) \bar{B}\left(\chi_{\eta}, \chi_{\eta}, 0\right)\left[1 / 9 \bar{\chi}_{1} R_{11}^{\eta} \chi_{1}-5 / 18 R_{11}^{\eta} \chi_{\eta} \chi_{1}\right] \\
& +2 / 9 \bar{A}\left(\chi_{\eta}\right) \bar{B}\left(\chi_{1}, \chi_{\eta}, 0\right)\left[R_{11}^{\eta} R_{\eta \eta}^{1} \chi_{1}^{2}-R_{14}^{\eta} R_{\eta \eta}^{1} \chi_{\eta} \chi_{1}\right]+\bar{A}\left(\chi_{\eta}\right) \bar{B}\left(\chi_{1}, \chi_{1}, 0\right)\left[2 / 9 R_{11}^{\eta} R_{1}^{c} \chi_{1}^{2}+1 / 9 R_{11}^{\eta} R_{1}^{d} \chi_{1}\right. \\
& \left.-1 / 27 R_{s s}^{\eta}\left(R_{s \eta}^{1}\right)^{2} \chi_{1} \chi_{s}\right]+2 / 9 \bar{A}\left(\chi_{\eta}\right) \bar{C}\left(\chi_{1}, \chi_{1}, \chi_{1}, 0\right) R_{11}^{\eta} R_{1}^{d} \chi_{1}^{2}+\bar{A}\left(\chi_{\eta}, \varepsilon\right) \pi_{16}\left[5 / 9 R_{11}^{\eta} \chi_{1}^{2}-1 / 4 R_{1}^{c} \chi_{\eta} \chi_{1}\right] \\
& -8 \bar{A}\left(\chi_{1}\right) L_{0}^{r}\left[R_{1}^{c} \chi_{1}^{2}+R_{1}^{d} \chi_{1}\right]+8 \bar{A}\left(\chi_{1}\right) L_{1}^{r} \chi_{1}^{2}+20 \bar{A}\left(\chi_{1}\right) L_{2}^{r} \chi_{1}^{2}-8 \bar{A}\left(\chi_{1}\right) L_{3}^{r}\left[R_{1}^{c} \chi_{1}^{2}+R_{1}^{d} \chi_{1}\right] \\
& +16 \bar{A}\left(\chi_{1}\right) L_{4}^{r} \bar{\chi}_{1} R_{1}^{c} \chi_{1}+\bar{A}\left(\chi_{1}\right) L_{5}^{r}\left[32 / 3 R_{1}^{c} \chi_{1}^{2}+16 / 3 R_{1}^{d} \chi_{1}\right]+\bar{A}\left(\chi_{1}\right) L_{6}^{r}\left[32 \chi_{1}^{2}-16 \bar{\chi}_{1} R_{1}^{c} \chi_{1}\right] \\
& +32 \bar{A}\left(\chi_{1}\right) L_{7}^{r} R_{1}^{d} \chi_{1}-64 / 3 \bar{A}\left(\chi_{1}\right) L_{8}^{r} R_{1}^{c} \chi_{1}^{2}+\bar{A}\left(\chi_{1}\right)^{2}\left[1 / 2 \chi_{1}+1 / 18\left(R_{1}^{c}\right)^{2} \chi_{1}\right] \\
& +1 / 3 \bar{A}\left(\chi_{1}\right) \bar{A}\left(\chi_{46}\right) R_{11}^{\eta} \chi_{1}+4 / 9 \bar{A}\left(\chi_{1}\right) \bar{A}\left(\chi_{1 s}\right) R_{1 s}^{\eta} R_{1 s \eta}^{z} \chi_{1}+2 / 9 \bar{A}\left(\chi_{1}\right) \bar{B}\left(\chi_{1}, \chi_{\eta}, 0\right) R_{11}^{\eta} R_{1}^{c} \chi_{1}^{2} \\
& +\bar{A}\left(\chi_{1}\right) \bar{B}\left(\chi_{1}, \chi_{1}, 0\right)\left[\chi_{1}^{2}+2 / 9\left(R_{1}^{c}\right)^{2} \chi_{1}^{2}+1 / 9 R_{1}^{c} R_{1}^{d} \chi_{1}\right]+2 / 9 \bar{A}\left(\chi_{1}\right) \bar{C}\left(\chi_{1}, \chi_{1}, \chi_{1}, 0\right) R_{1}^{c} R_{1}^{d} \chi_{1}^{2} \\
& -\bar{A}\left(\chi_{1}, \varepsilon\right) \pi_{16}\left[31 / 18 \chi_{1}^{2}-29 / 36 R_{1}^{c} \chi_{1}^{2}-1 / 4 R_{1}^{d} \chi_{1}\right]-\bar{A}\left(\chi_{14}\right)^{2} \chi_{1}-\bar{A}\left(\chi_{14}\right) \bar{A}\left(\chi_{16}\right) \chi_{1}-1 / 4 \bar{A}\left(\chi_{16}\right)^{2} \chi_{1} \\
& +48 \bar{A}\left(\chi_{4}\right) L_{1}^{r} \chi_{1} \chi_{4}+12 \bar{A}\left(\chi_{4}\right) L_{2}^{r} \chi_{1} \chi_{4}-48 \bar{A}\left(\chi_{4}\right) L_{4}^{r} \chi_{1} \chi_{4}+48 \bar{A}\left(\chi_{4}\right) L_{6}^{r} \chi_{1} \chi_{4} \\
& -1 / 6 \bar{A}\left(\chi_{4}\right) \bar{B}\left(\chi_{\eta}, \chi_{\eta}, 0\right) R_{11}^{\eta} \chi_{1} \chi_{4}+2 / 3 \bar{A}\left(\chi_{4}\right) \bar{B}\left(\chi_{1}, \chi_{\eta}, 0\right) R_{14}^{\eta} R_{4 \eta}^{1} \chi_{1} \chi_{4} \\
& +1 / 3 \bar{A}\left(\chi_{4}\right) \bar{B}\left(\chi_{1}, \chi_{1}, 0\right)\left(R_{4 \eta}^{1}\right)^{2} \chi_{1} \chi_{4}-3 / 4 \bar{A}\left(\chi_{4}, \varepsilon\right) \pi_{16} \chi_{1} \chi_{4}+64 \bar{A}\left(\chi_{46}\right) L_{1}^{r} \chi_{1} \chi_{46}+16 \bar{A}\left(\chi_{46}\right) L_{2}^{r} \chi_{1} \chi_{46} \\
& \text { - } 64 \bar{A}\left(\chi_{46}\right) L_{4}^{r} \chi_{1} \chi_{46}+64 \bar{A}\left(\chi_{46}\right) L_{6}^{r} \chi_{1} \chi_{46}+4 / 9 \bar{A}\left(\chi_{46}\right) \bar{B}\left(\chi_{\eta}, \chi_{\eta}, 0\right) R_{11}^{\eta} \chi_{1} \chi_{46} \\
& -4 / 27 \bar{A}\left(\chi_{46}\right) \bar{B}\left(\chi_{1}, \chi_{\eta}, 0\right)\left[R_{1146}^{u \eta} \chi_{1}^{2}-R_{1146}^{w \eta} \chi_{1}\right]+4 / 9 \bar{A}\left(\chi_{46}\right) \bar{B}\left(\chi_{1}, \chi_{1}, 0\right) R_{\eta \eta}^{1} \chi_{1} \chi_{46} \\
& -\bar{A}\left(\chi_{46}, \varepsilon\right) \pi_{16} \chi_{1} \chi_{46}+\bar{A}\left(\chi_{1 s}\right) \pi_{16}\left[1 / 4 \chi_{1} \chi_{s}+3 / 4 \bar{\chi}_{1} \chi_{1}\right]+8 \bar{A}\left(\chi_{1 s}\right) L_{0}^{r} \chi_{1} \chi_{1 s}+20 \bar{A}\left(\chi_{1 s}\right) L_{3}^{r} \chi_{1} \chi_{1 s} \\
& -16 \bar{A}\left(\chi_{1 s}\right) L_{5}^{r} \chi_{1} \chi_{1 s}+32 \bar{A}\left(\chi_{1 s}\right) L_{8}^{r} \chi_{1} \chi_{1 s}-\bar{A}\left(\chi_{1 s}\right) \bar{B}\left(\chi_{1}, \chi_{\eta}, 0\right)\left[4 / 9 R_{1 s}^{\eta} \chi_{1} \chi_{1 s}+2 / 9 R_{1 s}^{\eta} \chi_{1}^{2}\right] \\
& -2 / 3 \bar{A}\left(\chi_{1 s}\right) \bar{B}\left(\chi_{1}, \chi_{1}, 0\right) R_{s \eta}^{1} \chi_{1} \chi_{s}-\bar{A}\left(\chi_{1 s}, \varepsilon\right) \pi_{16}\left[3 / 4 \chi_{1} \chi_{s}+5 / 6 \chi_{1}^{2}+3 / 4 \bar{\chi}_{1} \chi_{1}\right] \\
& +8 \bar{B}\left(\chi_{\eta}, \chi_{\eta}, 0\right) L_{4}^{r} \bar{\chi}_{1} R_{11}^{\eta} \chi_{\eta} \chi_{1}+8 / 3 \bar{B}\left(\chi_{\eta}, \chi_{\eta}, 0\right) L_{5}^{r} R_{11}^{\eta} \chi_{\eta}^{2} \chi_{1}-16 \bar{B}\left(\chi_{\eta}, \chi_{\eta}, 0\right) L_{6}^{r} \bar{\chi}_{1} R_{11}^{\eta} \chi_{\eta} \chi_{1} \\
& +16 \bar{B}\left(\chi_{\eta}, \chi_{\eta}, 0\right) L_{7}^{r}\left(R_{\eta 461}^{z}\right)^{2} \chi_{1}-16 / 3 \bar{B}\left(\chi_{\eta}, \chi_{\eta}, 0\right) L_{8}^{r}\left[R_{11}^{\eta} \chi_{\eta}^{2} \chi_{1}-\left(R_{\eta 461}^{z}\right)^{2} \chi_{1}\right] \\
& +32 \bar{B}\left(\chi_{1}, \chi_{\eta}, 0\right) L_{7}^{r} R_{\eta 461}^{z} R_{1}^{d} \chi_{1}+32 / 3 \bar{B}\left(\chi_{1}, \chi_{\eta}, 0\right) L_{8}^{r} R_{\eta 461}^{z} R_{1}^{d} \chi_{1} \\
& +2 / 9 \bar{B}\left(\chi_{1}, \chi_{\eta}, 0\right) \bar{B}\left(\chi_{1}, \chi_{1}, 0\right) R_{11}^{\eta} R_{1}^{d} \chi_{1}^{2}-8 \bar{B}\left(\chi_{1}, \chi_{1}, 0\right) L_{0}^{r} R_{1}^{d} \chi_{1}^{2}-8 \bar{B}\left(\chi_{1}, \chi_{1}, 0\right) L_{3}^{r} R_{1}^{d} \chi_{1}^{2} \\
& +\bar{B}\left(\chi_{1}, \chi_{1}, 0\right) L_{4}^{r}\left[8 \bar{\chi}_{1} R_{1}^{c} \chi_{1}^{2}+24 \bar{\chi}_{1} R_{1}^{d} \chi_{1}\right]+\bar{B}\left(\chi_{1}, \chi_{1}, 0\right) L_{5}^{r}\left[8 / 3 R_{1}^{c} \chi_{1}^{3}+16 R_{1}^{d} \chi_{1}^{2}\right] \\
& \text { - } \bar{B}\left(\chi_{1}, \chi_{1}, 0\right) L_{6}^{r}\left[16 \bar{\chi}_{1} R_{1}^{c} \chi_{1}^{2}+32 \bar{\chi}_{1} R_{1}^{d} \chi_{1}\right]+16 \bar{B}\left(\chi_{1}, \chi_{1}, 0\right) L_{7}^{r}\left(R_{1}^{d}\right)^{2} \chi_{1}-\bar{B}\left(\chi_{1}, \chi_{1}, 0\right) L_{8}^{r}\left[16 / 3 R_{1}^{c} \chi_{1}^{3}\right. \\
& \left.+32 R_{1}^{d} \chi_{1}^{2}-16 / 3\left(R_{1}^{d}\right)^{2} \chi_{1}\right]+\bar{B}\left(\chi_{1}, \chi_{1}, 0\right)^{2}\left[2 / 9 R_{1}^{c} R_{1}^{d} \chi_{1}^{2}+1 / 18\left(R_{1}^{d}\right)^{2} \chi_{1}\right] \\
& +2 / 9 \bar{B}\left(\chi_{1}, \chi_{1}, 0\right) \bar{C}\left(\chi_{1}, \chi_{1}, \chi_{1}, 0\right)\left(R_{1}^{d}\right)^{2} \chi_{1}^{2}+29 / 36 \bar{B}\left(\chi_{1}, \chi_{1}, 0, \varepsilon\right) \pi_{16} R_{1}^{d} \chi_{1}^{2} \\
& +16 \bar{C}\left(\chi_{1}, \chi_{1}, \chi_{1}, 0\right) L_{4}^{r} \bar{\chi}_{1} R_{1}^{d} \chi_{1}^{2}+16 / 3 \bar{C}\left(\chi_{1}, \chi_{1}, \chi_{1}, 0\right) L_{5}^{r} R_{1}^{d} \chi_{1}^{3}-32 \bar{C}\left(\chi_{1}, \chi_{1}, \chi_{1}, 0\right) L_{6}^{r} \bar{\chi}_{1} R_{1}^{d} \chi_{1}^{2} \\
& -32 / 3 \bar{C}\left(\chi_{1}, \chi_{1}, \chi_{1}, 0\right) L_{8}^{r} R_{1}^{d} \chi_{1}^{3}+2 / 9 H^{F}\left(1, \chi_{\eta}, \chi_{\eta}, \chi_{1}, \chi_{1}\right)\left(R_{11}^{\eta}\right)^{2} \chi_{1}^{2}+4 / 9 H^{F}\left(1, \chi_{\eta}, \chi_{1}, \chi_{1}, \chi_{1}\right) R_{11}^{\eta} R_{1}^{c} \chi_{1}^{2} \\
& -H^{F}\left(1, \chi_{\eta}, \chi_{1 s}, \chi_{1 s}, \chi_{1}\right)\left[1 / 3 R_{11}^{\eta} \chi_{1}^{2}+1 / 12 R_{\eta 1 s}^{v} \chi_{\eta} \chi_{1}\right]+H^{F}\left(1, \chi_{1}, \chi_{1}, \chi_{1}, \chi_{1}\right)\left[1 / 3 \chi_{1}^{2}+2 / 9\left(R_{1}^{c}\right)^{2} \chi_{1}^{2}\right] \\
& -H^{F}\left(1, \chi_{1}, \chi_{1 s}, \chi_{1 s}, \chi_{1}\right)\left[1 / 2 R_{s \eta}^{1} \chi_{1}^{2}-1 / 4 R_{1}^{c} \chi_{1}^{2}+1 / 12 R_{1}^{d} \chi_{1}\right]+3 / 4 H^{F}\left(1, \chi_{14}, \chi_{14}, \chi_{4}, \chi_{1}\right) \chi_{1} \chi_{4} \\
& +H^{F}\left(1, \chi_{14}, \chi_{16}, \chi_{46}, \chi_{1}\right) \chi_{1} \chi_{46}+4 / 9 H^{F}\left(2, \chi_{1}, \chi_{\eta}, \chi_{1}, \chi_{1}\right) R_{11}^{\eta} R_{1}^{d} \chi_{1}^{2}+4 / 9 H^{F}\left(2, \chi_{1}, \chi_{1}, \chi_{1}, \chi_{1}\right) R_{1}^{c} R_{1}^{d} \chi_{1}^{2} \\
& +1 / 4 H^{F}\left(2, \chi_{1}, \chi_{1 s}, \chi_{1 s}, \chi_{1}\right) R_{1}^{d} \chi_{1}^{2}+2 / 9 H^{F}\left(5, \chi_{1}, \chi_{1}, \chi_{1}, \chi_{1}\right)\left(R_{1}^{d}\right)^{2} \chi_{1}^{2} \\
& -2 / 3 H_{1}^{F}\left(1, \chi_{\eta}, \chi_{1 s}, \chi_{1 s}, \chi_{1}\right) R_{1 s}^{\eta} R_{1 s \eta}^{z} \chi_{1}^{2}-4 / 3 H_{1}^{F}\left(1, \chi_{1 s}, \chi_{1 s}, \chi_{1}, \chi_{1}\right) R_{1 s}^{\eta} R_{1 s \eta}^{z} \chi_{1}^{2} \\
& -4 / 3 H_{1}^{F}\left(3, \chi_{1 s}, \chi_{1}, \chi_{1 s}, \chi_{1}\right) R_{1}^{d} \chi_{1}^{2}-1 / 4 H_{21}^{F}\left(1, \chi_{\eta}, \chi_{1 s}, \chi_{1 s}, \chi_{1}\right) R_{\eta 1 s}^{v} \chi_{1}^{2} \\
& +H_{21}^{F}\left(1, \chi_{1}, \chi_{1 s}, \chi_{1 s}, \chi_{1}\right)\left[1 / 2 R_{s \eta}^{1} \chi_{1}^{2}-1 / 4 R_{1}^{c} \chi_{1}^{2}\right]+9 / 4 H_{21}^{F}\left(1, \chi_{4}, \chi_{14}, \chi_{14}, \chi_{1}\right) \chi_{1}^{2}
\end{aligned}
$$


$+3 H_{21}^{F}\left(1, \chi_{46}, \chi_{14}, \chi_{16}, \chi_{1}\right) \chi_{1}^{2}-1 / 4 H_{21}^{F}\left(2, \chi_{1}, \chi_{1 s}, \chi_{1 s}, \chi_{1}\right) R_{1}^{d} \chi_{1}^{2}$,

$$
\begin{aligned}
& \delta_{\text {loops }}^{(6) 13}=\pi_{16} L_{0}^{r}\left[-8 / 9 \chi_{\pi} \chi_{\eta} \chi_{1}-\chi_{1}^{3}+26 / 3 \bar{\chi}_{1} \chi_{1}^{2}+35 / 9 \bar{\chi}_{2} \chi_{1}\right]+4 \pi_{16} L_{1}^{r} \chi_{1}^{3}+\pi_{16} L_{2}^{r}\left[22 / 3 \chi_{\pi} \chi_{\eta} \chi_{1}+2 \chi_{1}^{3}\right. \\
& \left.+26 / 3 \bar{\chi}_{2} \chi_{1}\right]+\pi_{16} L_{3}^{r}\left[-8 / 9 \chi_{\pi} \chi_{\eta} \chi_{1}-5 / 2 \chi_{1}^{3}+17 / 3 \bar{\chi}_{1} \chi_{1}^{2}+43 / 18 \bar{\chi}_{2} \chi_{1}\right]+\pi_{16}^{2}\left[15 / 32 \chi_{\pi} \chi_{\eta} \chi_{1}\right. \\
& \left.-3 / 32 \chi_{1}^{3}+15 / 32 \bar{\chi}_{1} \chi_{1}^{2}+43 / 64 \bar{\chi}_{2} \chi_{1}\right]+384 L_{4}^{r} L_{5}^{r} \bar{\chi}_{1} \chi_{1}^{2}-1152 L_{4}^{r} L_{6}^{r} \bar{\chi}_{1}^{2} \chi_{1}-384 L_{4}^{r} L_{8}^{r} \bar{\chi}_{1} \chi_{1}^{2} \\
& +576 L_{4}^{r 2} \bar{\chi}_{1}^{2} \chi_{1}-384 L_{5}^{r} L_{6}^{r} \bar{\chi}_{1} \chi_{1}^{2}-128 L_{5}^{r} L_{8}^{r} \chi_{1}^{3}+64 L_{5}^{r 2} \chi_{1}^{3}-8 \bar{A}\left(\chi_{m}\right) L_{0}^{r} R_{n 11}^{m} \chi_{m} \chi_{1} \\
& +16 \bar{A}\left(\chi_{m}\right) L_{1}^{r} \chi_{m} \chi_{1}+4 \bar{A}\left(\chi_{m}\right) L_{2}^{r} \chi_{m} \chi_{1}-8 \bar{A}\left(\chi_{m}\right) L_{3}^{r} R_{n 11}^{m} \chi_{m} \chi_{1}-16 \bar{A}\left(\chi_{m}\right) L_{4}^{r}\left[\chi_{m} \chi_{1}-\bar{\chi}_{1} R_{n 11}^{m} \chi_{1}\right] \\
& +16 / 3 \bar{A}\left(\chi_{m}\right) L_{5}^{r}\left[R_{n 11}^{m} \chi_{m} \chi_{1}+R_{n 11}^{m} \chi_{1}^{2}\right]+16 \bar{A}\left(\chi_{m}\right) L_{6}^{r}\left[\chi_{m} \chi_{1}-\bar{\chi}_{1} R_{n 11}^{m} \chi_{1}\right]+32 \bar{A}\left(\chi_{m}\right) L_{7}^{r} R_{m 456 n 1}^{z} \chi_{1} \\
& -64 / 3 \bar{A}\left(\chi_{m}\right) L_{8}^{r} R_{n 11}^{m} \chi_{1}^{2}+1 / 18 \bar{A}\left(\chi_{m}\right)^{2}\left(R_{n 11}^{m}\right)^{2} \chi_{1}+1 / 9 \bar{A}\left(\chi_{m}\right) \bar{A}\left(\chi_{1}\right) R_{n 11}^{m} R_{1}^{c} \chi_{1} \\
& -4 / 9 \bar{A}\left(\chi_{m}\right) \bar{A}\left(\chi_{1 s}\right) R_{n 1 s}^{m} R_{1 s m}^{z} \chi_{1}+\bar{A}\left(\chi_{m}\right) \bar{A}\left(\chi_{s t}\right)\left[1 / 27 R_{n 11}^{m} R_{m n s t}^{v} \chi_{1}+2 / 27 R_{n 1 s}^{m} R_{m 1 s}^{n} R_{t s s m n}^{z} \chi_{1}\right] \\
& -1 / 27 \bar{A}\left(\chi_{m}\right) \bar{B}\left(\chi_{m}, \chi_{m}, 0\right)\left(R_{n 1 s}^{m}\right)^{2} R_{n s s}^{m} \chi_{1} \chi_{s}-2 / 27 \bar{A}\left(\chi_{m}\right) \bar{B}\left(\chi_{m}, \chi_{n}, 0\right) R_{n 1 s}^{m} R_{n s s}^{m} R_{m 1 s}^{n} \chi_{1} \chi_{s} \\
& +\bar{A}\left(\chi_{m}\right) \bar{B}\left(\chi_{m}, \chi_{1}, 0\right)\left[2 / 9\left(R_{n 11}^{m}\right)^{2} \chi_{1}^{2}-2 / 27 R_{n 1 s}^{m} R_{n s s}^{m} R_{s m n}^{1} \chi_{1} \chi_{s}\right] \\
& \text { - } 1 / 27 \bar{A}\left(\chi_{m}\right) \bar{B}\left(\chi_{n}, \chi_{n}, 0\right) R_{n s s}^{m}\left(R_{m 1 s}^{n}\right)^{2} \chi_{1} \chi_{s}-\bar{A}\left(\chi_{m}\right) \bar{B}\left(\chi_{n}, \chi_{1}, 0\right)\left[2 / 27 R_{n s s}^{m} R_{m 1 s}^{n} R_{s m n}^{1} \chi_{1} \chi_{s}\right. \\
& \left.-2 / 9 R_{\eta 11}^{\pi} R_{\pi 11}^{\eta} \chi_{1}^{2}\right]+\bar{A}\left(\chi_{m}\right) \bar{B}\left(\chi_{1}, \chi_{1}, 0\right)\left[2 / 9 R_{n 11}^{m} R_{1}^{c} \chi_{1}^{2}+1 / 9 R_{n 11}^{m} R_{1}^{d} \chi_{1}-1 / 27 R_{n s s}^{m}\left(R_{s m n}^{1}\right)^{2} \chi_{1} \chi_{s}\right] \\
& +2 / 9 \bar{A}\left(\chi_{m}\right) \bar{C}\left(\chi_{1}, \chi_{1}, \chi_{1}, 0\right) R_{n 11}^{m} R_{1}^{d} \chi_{1}^{2}+\bar{A}\left(\chi_{m}, \varepsilon\right) \pi_{16}\left[-1 / 4 \chi_{m} \chi_{1}+1 / 4 R_{n 11}^{m} \chi_{m} \chi_{1}+5 / 9 R_{n 11}^{m} \chi_{1}^{2}\right] \\
& +1 / 9 \bar{A}\left(\chi_{\pi}\right) \bar{A}\left(\chi_{\eta}\right) R_{\eta 11}^{\pi} R_{\pi 11}^{\eta} \chi_{1}-8 \bar{A}\left(\chi_{1}\right) L_{0}^{r}\left[R_{1}^{c} \chi_{1}^{2}+R_{1}^{d} \chi_{1}\right]+8 \bar{A}\left(\chi_{1}\right) L_{1}^{r} \chi_{1}^{2} \\
& +20 \bar{A}\left(\chi_{1}\right) L_{2}^{r} \chi_{1}^{2}-8 \bar{A}\left(\chi_{1}\right) L_{3}^{r}\left[R_{1}^{c} \chi_{1}^{2}+R_{1}^{d} \chi_{1}\right]+16 \bar{A}\left(\chi_{1}\right) L_{4}^{r} \bar{\chi}_{1} R_{1}^{c} \chi_{1} \\
& +\bar{A}\left(\chi_{1}\right) L_{5}^{r}\left[32 / 3 R_{1}^{c} \chi_{1}^{2}+16 / 3 R_{1}^{d} \chi_{1}\right]+\bar{A}\left(\chi_{1}\right) L_{6}^{r}\left[32 \chi_{1}^{2}-16 \bar{\chi}_{1} R_{1}^{c} \chi_{1}\right]+32 \bar{A}\left(\chi_{1}\right) L_{7}^{r} R_{1}^{d} \chi_{1} \\
& \text { - 64/3 } \bar{A}\left(\chi_{1}\right) L_{8}^{r} R_{1}^{c} \chi_{1}^{2}+\bar{A}\left(\chi_{1}\right)^{2}\left[1 / 2 \chi_{1}+1 / 18\left(R_{1}^{c}\right)^{2} \chi_{1}\right]+4 / 9 \bar{A}\left(\chi_{1}\right) \bar{A}\left(\chi_{1 s}\right) R_{n 1 s}^{m} R_{1 s m}^{z} \chi_{1} \\
& -1 / 27 \bar{A}\left(\chi_{1}\right) \bar{A}\left(\chi_{s t}\right)\left(R_{s \pi \eta}^{1}\right)^{2} R_{t s s 11}^{z} \chi_{1}+2 / 9 \bar{A}\left(\chi_{1}\right) \bar{B}\left(\chi_{m}, \chi_{1}, 0\right) R_{n 11}^{m} R_{1}^{c} \chi_{1}^{2}+\bar{A}\left(\chi_{1}\right) \bar{B}\left(\chi_{1}, \chi_{1}, 0\right)\left[\chi_{1}^{2}\right. \\
& \left.+2 / 9\left(R_{1}^{c}\right)^{2} \chi_{1}^{2}+1 / 9 R_{1}^{c} R_{1}^{d} \chi_{1}\right]+2 / 9 \bar{A}\left(\chi_{1}\right) \bar{C}\left(\chi_{1}, \chi_{1}, \chi_{1}, 0\right) R_{1}^{c} R_{1}^{d} \chi_{1}^{2}+\bar{A}\left(\chi_{1}, \varepsilon\right) \pi_{16}\left[-31 / 18 \chi_{1}^{2}\right. \\
& \left.+29 / 36 R_{1}^{c} \chi_{1}^{2}+1 / 4 R_{1}^{d} \chi_{1}\right]+\bar{A}\left(\chi_{1 s}\right) \pi_{16}\left[1 / 4 \chi_{1} \chi_{s}+3 / 4 \bar{\chi}_{1} \chi_{1}\right]+8 \bar{A}\left(\chi_{1 s}\right) L_{0}^{r} \chi_{1} \chi_{1 s} \\
& +20 \bar{A}\left(\chi_{1 s}\right) L_{3}^{r} \chi_{1} \chi_{1 s}-16 \bar{A}\left(\chi_{1 s}\right) L_{5}^{r} \chi_{1} \chi_{1 s}+32 \bar{A}\left(\chi_{1 s}\right) L_{8}^{r} \chi_{1} \chi_{1 s}-1 / 4 \bar{A}\left(\chi_{1 s}\right)^{2} \chi_{1} \\
& \text { - } 1 / 2 \bar{A}\left(\chi_{1 s}\right) \bar{A}\left(\chi_{1 t}\right) \chi_{1}-\bar{A}\left(\chi_{1 s}\right) \bar{B}\left(\chi_{m}, \chi_{1}, 0\right)\left[4 / 9 R_{n 1 s}^{m} \chi_{1} \chi_{1 s}+2 / 9 R_{n 1 s}^{m} \chi_{1}^{2}\right] \\
& -2 / 3 \bar{A}\left(\chi_{1 s}\right) \bar{B}\left(\chi_{1}, \chi_{1}, 0\right) R_{s \pi \eta}^{1} \chi_{1} \chi_{s}-\bar{A}\left(\chi_{1 s}, \varepsilon\right) \pi_{16}\left[3 / 4 \chi_{1} \chi_{s}+5 / 6 \chi_{1}^{2}+3 / 4 \bar{\chi}_{1} \chi_{1}\right]+32 \bar{A}\left(\chi_{s t}\right) L_{1}^{r} \chi_{1} \chi_{s t} \\
& +8 \bar{A}\left(\chi_{s t}\right) L_{2}^{r} \chi_{1} \chi_{s t}-32 \bar{A}\left(\chi_{s t}\right) L_{4}^{r} \chi_{1} \chi_{s t}+32 \bar{A}\left(\chi_{s t}\right) L_{6}^{r} \chi_{1} \chi_{s t}+2 / 9 \bar{A}\left(\chi_{s t}\right) \bar{B}\left(\chi_{m}, \chi_{m}, 0\right) R_{n 11}^{m} R_{n s t}^{m} \chi_{1} \chi_{s t} \\
& -2 / 27 \bar{A}\left(\chi_{s t}\right) \bar{B}\left(\chi_{m}, \chi_{1}, 0\right)\left[R_{11 s t}^{u m} \chi_{1}^{2}-R_{11 s t}^{w m} \chi_{1}\right]+2 / 27 \bar{A}\left(\chi_{s t}\right) \bar{B}\left(\chi_{\pi}, \chi_{\eta}, 0\right) R_{11 s t}^{w \pi \eta} \chi_{1} \\
& -2 / 27 \bar{A}\left(\chi_{s t}\right) \bar{B}\left(\chi_{\pi}, \chi_{\eta}, 0, k\right) R_{\eta 1 s}^{\pi} R_{\pi 1 s}^{\eta} R_{t s s \pi \eta}^{z} \chi_{1}+2 / 9 \bar{A}\left(\chi_{s t}\right) \bar{B}\left(\chi_{1}, \chi_{1}, 0\right) R_{s \eta \eta}^{1} R_{t \pi \pi}^{1} \chi_{1} \chi_{s t} \\
& -1 / 2 \bar{A}\left(\chi_{s t}, \varepsilon\right) \pi_{16} \chi_{1} \chi_{s t}+8 \bar{B}\left(\chi_{m}, \chi_{m}, 0\right) L_{4}^{r} \bar{\chi}_{1} R_{n 11}^{m} \chi_{m} \chi_{1}+8 / 3 \bar{B}\left(\chi_{m}, \chi_{m}, 0\right) L_{5}^{r} R_{n 11}^{m} \chi_{m}^{2} \chi_{1} \\
& \text { - } 16 \bar{B}\left(\chi_{m}, \chi_{m}, 0\right) L_{6}^{r} \bar{\chi}_{1} R_{n 11}^{m} \chi_{m} \chi_{1}+16 \bar{B}\left(\chi_{m}, \chi_{m}, 0\right) L_{7}^{r}\left(R_{m 456 n 1}^{z}\right)^{2} \chi_{1} \\
& \text { - 16/3 } \bar{B}\left(\chi_{m}, \chi_{m}, 0\right) L_{8}^{r}\left[R_{n 11}^{m} \chi_{m}^{2} \chi_{1}-\left(R_{m 456 n 1}^{z}\right)^{2} \chi_{1}\right]+32 \bar{B}\left(\chi_{m}, \chi_{1}, 0\right) L_{7}^{r} R_{m 456 n 1}^{z} R_{1}^{d} \chi_{1} \\
& +32 / 3 \bar{B}\left(\chi_{m}, \chi_{1}, 0\right) L_{8}^{r} R_{m 456 n 1}^{z} R_{1}^{d} \chi_{1}+2 / 9 \bar{B}\left(\chi_{m}, \chi_{1}, 0\right) \bar{B}\left(\chi_{1}, \chi_{1}, 0\right) R_{n 11}^{m} R_{1}^{d} \chi_{1}^{2} \\
& +32 \bar{B}\left(\chi_{\pi}, \chi_{\eta}, 0\right) L_{7}^{r} R_{\pi 456 \eta 1}^{z} R_{\eta 456 \pi 1}^{z} \chi_{1}+32 / 3 \bar{B}\left(\chi_{\pi}, \chi_{\eta}, 0\right) L_{8}^{r} R_{\pi 456 \eta 1}^{z} R_{\eta 456 \pi 1}^{z} \chi_{1}-8 \bar{B}\left(\chi_{1}, \chi_{1}, 0\right) L_{0}^{r} R_{1}^{d} \chi_{1}^{2} \\
& -8 \bar{B}\left(\chi_{1}, \chi_{1}, 0\right) L_{3}^{r} R_{1}^{d} \chi_{1}^{2}+\bar{B}\left(\chi_{1}, \chi_{1}, 0\right) L_{4}^{r}\left[8 \bar{\chi}_{1} R_{1}^{c} \chi_{1}^{2}+24 \bar{\chi}_{1} R_{1}^{d} \chi_{1}\right]+\bar{B}\left(\chi_{1}, \chi_{1}, 0\right) L_{5}^{r}\left[8 / 3 R_{1}^{c} \chi_{1}^{3}\right. \\
& \left.+16 R_{1}^{d} \chi_{1}^{2}\right]-\bar{B}\left(\chi_{1}, \chi_{1}, 0\right) L_{6}^{r}\left[16 \bar{\chi}_{1} R_{1}^{c} \chi_{1}^{2}+32 \bar{\chi}_{1} R_{1}^{d} \chi_{1}\right]+16 \bar{B}\left(\chi_{1}, \chi_{1}, 0\right) L_{7}^{r}\left(R_{1}^{d}\right)^{2} \chi_{1} \\
& \text { - } \bar{B}\left(\chi_{1}, \chi_{1}, 0\right) L_{8}^{r}\left[16 / 3 R_{1}^{c} \chi_{1}^{3}+32 R_{1}^{d} \chi_{1}^{2}-16 / 3\left(R_{1}^{d}\right)^{2} \chi_{1}\right]+\bar{B}\left(\chi_{1}, \chi_{1}, 0\right)^{2}\left[2 / 9 R_{1}^{c} R_{1}^{d} \chi_{1}^{2}+1 / 18\left(R_{1}^{d}\right)^{2} \chi_{1}\right] \\
& +2 / 9 \bar{B}\left(\chi_{1}, \chi_{1}, 0\right) \bar{C}\left(\chi_{1}, \chi_{1}, \chi_{1}, 0\right)\left(R_{1}^{d}\right)^{2} \chi_{1}^{2}+29 / 36 \bar{B}\left(\chi_{1}, \chi_{1}, 0, \varepsilon\right) \pi_{16} R_{1}^{d} \chi_{1}^{2} \\
& +16 \bar{C}\left(\chi_{1}, \chi_{1}, \chi_{1}, 0\right) L_{4}^{r} \bar{\chi}_{1} R_{1}^{d} \chi_{1}^{2}+16 / 3 \bar{C}\left(\chi_{1}, \chi_{1}, \chi_{1}, 0\right) L_{5}^{r} R_{1}^{d} \chi_{1}^{3}-32 \bar{C}\left(\chi_{1}, \chi_{1}, \chi_{1}, 0\right) L_{6}^{r} \bar{\chi}_{1} R_{1}^{d} \chi_{1}^{2} \\
& -32 / 3 \bar{C}\left(\chi_{1}, \chi_{1}, \chi_{1}, 0\right) L_{8}^{r} R_{1}^{d} \chi_{1}^{3}+2 / 9 H^{F}\left(1, \chi_{m}, \chi_{m}, \chi_{1}, \chi_{1}\right)\left(R_{n 11}^{m}\right)^{2} \chi_{1}^{2} \\
& +4 / 9 H^{F}\left(1, \chi_{m}, \chi_{1}, \chi_{1}, \chi_{1}\right) R_{n 11}^{m} R_{1}^{c} \chi_{1}^{2}-H^{F}\left(1, \chi_{m}, \chi_{1 s}, \chi_{1 s}, \chi_{1}\right)\left[1 / 3 R_{n 11}^{m} \chi_{1}^{2}+1 / 12 R_{m n 1 s}^{v} \chi_{m} \chi_{1}\right] \\
& +4 / 9 H^{F}\left(1, \chi_{\pi}, \chi_{\eta}, \chi_{1}, \chi_{1}\right) R_{\eta 11}^{\pi} R_{\pi 11}^{\eta} \chi_{1}^{2}+H^{F}\left(1, \chi_{1}, \chi_{1}, \chi_{1}, \chi_{1}\right)\left[1 / 3 \chi_{1}^{2}+2 / 9\left(R_{1}^{c}\right)^{2} \chi_{1}^{2}\right] \\
& -H^{F}\left(1, \chi_{1}, \chi_{1 s}, \chi_{1 s}, \chi_{1}\right)\left[1 / 2 R_{s \pi \eta}^{1} \chi_{1}^{2}-1 / 4 R_{1}^{c} \chi_{1}^{2}+1 / 12 R_{1}^{d} \chi_{1}\right]+1 / 2 H^{F}\left(1, \chi_{1 s}, \chi_{1 t}, \chi_{s t}, \chi_{1}\right) \chi_{1} \chi_{s t}
\end{aligned}
$$




$$
\begin{aligned}
& +4 / 9 H^{F}\left(2, \chi_{1}, \chi_{m}, \chi_{1}, \chi_{1}\right) R_{n 11}^{m} R_{1}^{d} \chi_{1}^{2}+4 / 9 H^{F}\left(2, \chi_{1}, \chi_{1}, \chi_{1}, \chi_{1}\right) R_{1}^{c} R_{1}^{d} \chi_{1}^{2} \\
& +1 / 4 H^{F}\left(2, \chi_{1}, \chi_{1 s}, \chi_{1 s}, \chi_{1}\right) R_{1}^{d} \chi_{1}^{2}+2 / 9 H^{F}\left(5, \chi_{1}, \chi_{1}, \chi_{1}, \chi_{1}\right)\left(R_{1}^{d}\right)^{2} \chi_{1}^{2} \\
& -2 / 3 H_{1}^{F}\left(1, \chi_{m}, \chi_{1 s}, \chi_{1 s}, \chi_{1}\right) R_{n 1 s}^{m} R_{1 s m}^{z} \chi_{1}^{2}-4 / 3 H_{1}^{F}\left(1, \chi_{1 s}, \chi_{1 s}, \chi_{1}, \chi_{1}\right) R_{n 1 s}^{m} R_{1 s m}^{z} \chi_{1}^{2} \\
& -4 / 3 H_{1}^{F}\left(3, \chi_{1 s}, \chi_{1}, \chi_{1 s}, \chi_{1}\right) R_{1}^{d} \chi_{1}^{2}-1 / 4 H_{21}^{F}\left(1, \chi_{m}, \chi_{1 s}, \chi_{1 s}, \chi_{1}\right) R_{m n 1 s}^{v} \chi_{1}^{2} \\
& +H_{21}^{F}\left(1, \chi_{1}, \chi_{1 s}, \chi_{1 s}, \chi_{1}\right)\left[1 / 2 R_{s \pi \eta}^{1} \chi_{1}^{2}-1 / 4 R_{1}^{c} \chi_{1}^{2}\right]+3 / 2 H_{21}^{F}\left(1, \chi_{s t}, \chi_{1 s}, \chi_{1 t}, \chi_{1}\right) \chi_{1}^{2} \\
& -1 / 4 H_{21}^{F}\left(2, \chi_{1}, \chi_{1 s}, \chi_{1 s}, \chi_{1}\right) R_{1}^{d} \chi_{1}^{2} .
\end{aligned}
$$

\section{Results for $d_{\text {val }}=2$}

In general, the results for $d_{\mathrm{val}}=2$ are similar in form to those for $d_{\mathrm{val}}=1$. However, they are about twice as long, since the number of independent combinations of quark masses that can appear in the propagator residues and loop integrals is much larger. As already noted for $d_{\mathrm{val}}=1$, the expressions for $d_{\mathrm{val}}=2$ also become longer with increasing values of $d_{\text {sea }}$. The NLO expression for $d_{\text {val }}=2$ and $d_{\text {sea }}=3$, which agrees with the result of Refs. [3, 7], is

$$
\begin{aligned}
\delta^{(4) 23}= & -24 L_{4}^{r} \bar{\chi}_{1} \chi_{13}-8 L_{5}^{r} \chi_{13}^{2}+48 L_{6}^{r} \bar{\chi}_{1} \chi_{13} \\
& +16 L_{8}^{r} \chi_{13}^{2}-1 / 3 \bar{A}\left(\chi_{p}\right) R_{q \pi \eta}^{p} \chi_{13} \\
& -1 / 3 \bar{A}\left(\chi_{m}\right) R_{n 13}^{m} \chi_{13},
\end{aligned}
$$

while the contribution from the $\mathcal{O}\left(p^{6}\right)$ counterterms for $d_{\text {val }}=2$ and $d_{\text {sea }}=3$ is given by

$$
\begin{aligned}
\delta_{\mathrm{ct}}^{(6) 23}= & -32 K_{17}^{r} \chi_{13}^{3}-96 K_{18}^{r} \bar{\chi}_{1} \chi_{13}^{2}-8 K_{19}^{r} \chi_{p}^{2} \chi_{13} \\
& -48 K_{20}^{r} \bar{\chi}_{1} \chi_{13}^{2}-48 K_{21}^{r} \bar{\chi}_{2} \chi_{13} \\
& -144 K_{22}^{r} \bar{\chi}_{1}^{2} \chi_{13}-16 K_{23}^{r} \chi_{1} \chi_{13} \chi_{3} \\
& +24 K_{25}^{r} \chi_{p}^{2} \chi_{13}+K_{26}^{r}\left[96 \bar{\chi}_{1} \chi_{13}^{2}\right. \\
& \left.+48 \bar{\chi}_{2} \chi_{13}\right]+432 K_{27}^{r} \bar{\chi}_{1}^{2} \chi_{13}+32 K_{39}^{r} \chi_{13}^{3} \\
& +96 K_{40}^{r} \bar{\chi}_{1} \chi_{13}^{2} .
\end{aligned}
$$

Similarly to the case of $d_{\mathrm{val}}=1$, the expressions for other values of $d_{\text {sea }}$ can be straightforwardly obtained by taking the appropriate quark mass limits in the above expressions. Finally, the chiral loop contributions for $d_{\mathrm{val}}=2$ at NNLO are, for $d_{\text {sea }}=1, d_{\text {sea }}=2$, and $d_{\text {sea }}=3$, respectively,

$$
\begin{aligned}
& \delta_{\text {loops }}^{(6) 21}=\pi_{16} L_{0}^{r}\left[\chi_{1} \chi_{13} \chi_{3}+3 \chi_{13} \chi_{4}^{2}+26 / 3 \chi_{13}^{2} \chi_{4}-2 \chi_{13}^{3}\right]+4 \pi_{16} L_{1}^{r} \chi_{13}^{3}+\pi_{16} L_{2}^{r}\left[16 \chi_{13} \chi_{4}^{2}+2 \chi_{13}^{3}\right] \\
& +\pi_{16} L_{3}^{r}\left[7 / 6 \chi_{1} \chi_{13} \chi_{3}+3 / 2 \chi_{13} \chi_{4}^{2}+17 / 3 \chi_{13}^{2} \chi_{4}-11 / 3 \chi_{13}^{3}\right]+\pi_{16}^{2}\left[59 / 192 \chi_{1} \chi_{13} \chi_{3}+73 / 64 \chi_{13} \chi_{4}^{2}\right. \\
& \left.+15 / 32 \chi_{13}^{2} \chi_{4}-77 / 192 \chi_{13}^{3}\right]+384 L_{4}^{r} L_{5}^{r} \chi_{13}^{2} \chi_{4}-1152 L_{4}^{r} L_{6}^{r} \chi_{13} \chi_{4}^{2}-384 L_{4}^{r} L_{8}^{r} \chi_{13}^{2} \chi_{4}+576 L_{4}^{r 2} \chi_{13} \chi_{4}^{2} \\
& -384 L_{5}^{r} L_{6}^{r} \chi_{13}^{2} \chi_{4}-128 L_{5}^{r} L_{8}^{r} \chi_{13}^{3}+64 L_{5}^{r 2} \chi_{13}^{3}-\bar{A}\left(\chi_{p}\right) \pi_{16}\left[1 / 24 \chi_{q} \chi_{13}+1 / 8 \chi_{13} \chi_{4}-1 / 4 R_{q}^{p} \chi_{13} \chi_{4}\right. \\
& \left.-1 / 12 R_{q}^{p} \chi_{13}^{2}\right]-\bar{A}\left(\chi_{p}\right) L_{0}^{r}\left[4 / 3 \chi_{p} \chi_{13}+16 / 3 R_{q}^{p} \chi_{p} \chi_{13}+4 / 3 R_{p}^{d} \chi_{13}\right]-\bar{A}\left(\chi_{p}\right) L_{3}^{r}\left[10 / 3 \chi_{p} \chi_{13}\right. \\
& \left.+4 / 3 R_{q}^{p} \chi_{p} \chi_{13}+10 / 3 R_{p}^{d} \chi_{13}\right]+16 \bar{A}\left(\chi_{p}\right) L_{4}^{r} R_{q}^{p} \chi_{13} \chi_{4}+\bar{A}\left(\chi_{p}\right) L_{5}^{r}\left[8 / 3 \chi_{p} \chi_{13}+16 / 3 R_{q}^{p} \chi_{p} \chi_{13}\right] \\
& -16 \bar{A}\left(\chi_{p}\right) L_{6}^{r} R_{q}^{p} \chi_{13} \chi_{4}+\bar{A}\left(\chi_{p}\right) L_{7}^{r}\left[16 / 3 R_{p}^{d} \chi_{p 4}+32 / 3 R_{p}^{d} \chi_{13}\right]-\bar{A}\left(\chi_{p}\right) L_{8}^{r}\left[16 / 3 \chi_{p} \chi_{13}+32 / 9 R_{q}^{p} \chi_{1} \chi_{3}\right. \\
& \left.+64 / 9 R_{q}^{p} \chi_{13}^{2}+8 / 9\left(R_{p}^{d}\right)^{2}\right]+\bar{A}\left(\chi_{p}\right)^{2}\left[5 / 36 \chi_{13}+1 / 18\left(R_{q}^{p}\right)^{2} \chi_{13}-1 / 54 R_{p}^{q} \chi_{p}\right]+\bar{A}\left(\chi_{p}\right) \bar{A}\left(\chi_{p 4}\right)\left[1 / 24 \chi_{p}\right. \\
& \left.-1 / 8 \chi_{q}+1 / 24 R_{q}^{p} \chi_{p}+19 / 72 R_{q}^{p} \chi_{q}\right]+\bar{A}\left(\chi_{p}\right) \bar{A}\left(\chi_{q 4}\right)\left[1 / 4 \chi_{13}+1 / 6 R_{q}^{p} \chi_{q}-1 / 24 R_{p}^{q} \chi_{p}+1 / 72 R_{p}^{q} \chi_{q}\right] \\
& +\bar{A}\left(\chi_{p}\right) \bar{A}\left(\chi_{13}\right)\left[1 / 18 \chi_{p}-1 / 36 \chi_{13}\right]-4 / 27 \bar{A}\left(\chi_{p}\right) \bar{A}\left(\chi_{4}\right) \chi_{4}+\bar{A}\left(\chi_{p}\right) \bar{B}\left(\chi_{p}, \chi_{p}, 0\right)\left[11 / 54 R_{q}^{p} \chi_{p} \chi_{13}\right. \\
& \left.-1 / 27 R_{q}^{p} \chi_{p}^{2}-1 / 18 R_{q}^{p} \chi_{13}^{2}+1 / 54 R_{q}^{p} R_{p}^{d} \chi_{p}\right]-\bar{A}\left(\chi_{p}\right) \bar{B}\left(\chi_{q}, \chi_{q}, 0\right)\left[1 / 54 R_{q}^{p} R_{q}^{d} \chi_{q}+1 / 36 R_{q}^{d} \chi_{13}\right] \\
& +2 / 27 \bar{A}\left(\chi_{p}\right) \bar{B}\left(\chi_{1}, \chi_{3}, 0\right) R_{p}^{q} \chi_{p} \chi_{13}+1 / 27 \bar{A}\left(\chi_{p}\right) \bar{B}\left(\chi_{1}, \chi_{3}, 0, k\right) R_{p}^{q} \chi_{p}+\bar{A}\left(\chi_{p}, \varepsilon\right) \pi_{16}\left[13 / 72 \chi_{p} \chi_{13}\right. \\
& \left.+5 / 72 \chi_{q} \chi_{13}+5 / 36 \chi_{13} \chi_{4}+5 / 18 R_{q}^{p} \chi_{p} \chi_{13}-1 / 4 R_{q}^{p} \chi_{13} \chi_{4}\right]-\bar{A}\left(\chi_{p 4}\right) \pi_{16}\left[3 / 4 \chi_{p} \chi_{13}-3 / 2 \chi_{13} \chi_{4}\right. \\
& \left.-3 / 4 \chi_{13}^{2}\right]+12 \bar{A}\left(\chi_{p 4}\right) L_{0}^{r} \chi_{p 4} \chi_{13}+30 \bar{A}\left(\chi_{p 4}\right) L_{3}^{r} \chi_{p 4} \chi_{13}-24 \bar{A}\left(\chi_{p 4}\right) L_{5}^{r} \chi_{p 4} \chi_{13}+48 \bar{A}\left(\chi_{p 4}\right) L_{8}^{r} \chi_{p 4} \chi_{13} \\
& +1 / 4 \bar{A}\left(\chi_{p 4}\right) \bar{B}\left(\chi_{q}, \chi_{q}, 0\right) R_{q}^{d} \chi_{13}-\bar{A}\left(\chi_{p 4}\right) \bar{B}\left(\chi_{1}, \chi_{3}, 0\right)\left[4 / 9 \chi_{p} \chi_{13}+2 / 9 \chi_{13} \chi_{4}-1 / 9 R_{q}^{p} \chi_{p}^{2}\right] \\
& -\bar{A}\left(\chi_{p 4}\right) \bar{B}\left(\chi_{1}, \chi_{3}, 0, k\right)\left[2 / 9 \chi_{p 4}+2 / 9 \chi_{13}-1 / 9 R_{p}^{q} \chi_{q}\right]-\bar{A}\left(\chi_{p 4}, \varepsilon\right) \pi_{16}\left[9 / 4 \chi_{13} \chi_{4}+5 / 4 \chi_{13}^{2}\right] \\
& -\bar{A}\left(\chi_{1}\right) \bar{A}\left(\chi_{3}\right)\left[1 / 36 \chi_{13}+1 / 54 \chi_{4}-1 / 9 R_{3}^{1} R_{1}^{3} \chi_{13}\right]+8 \bar{A}\left(\chi_{13}\right) L_{1}^{r} \chi_{13}^{2}+20 \bar{A}\left(\chi_{13}\right) L_{2}^{r} \chi_{13}^{2} \\
& +32 \bar{A}\left(\chi_{13}\right) L_{6}^{r} \chi_{13}^{2}-1 / 4 \bar{A}\left(\chi_{13}\right)^{2} \chi_{13}+\bar{A}\left(\chi_{13}\right) \bar{B}\left(\chi_{1}, \chi_{3}, 0\right)\left[1 / 9 \chi_{1} \chi_{3}+4 / 9 \chi_{13}^{2}\right]
\end{aligned}
$$


$+4 / 9 \bar{A}\left(\chi_{13}\right) \bar{B}\left(\chi_{1}, \chi_{3}, 0, k\right) \chi_{13}-31 / 18 \bar{A}\left(\chi_{13}, \varepsilon\right) \pi_{16} \chi_{13}^{2}-9 / 4 \bar{A}\left(\chi_{14}\right) \bar{A}\left(\chi_{34}\right) \chi_{13}+128 \bar{A}\left(\chi_{4}\right) L_{1}^{r} \chi_{13} \chi_{4}$ $+32 \bar{A}\left(\chi_{4}\right) L_{2}^{r} \chi_{13} \chi_{4}-128 \bar{A}\left(\chi_{4}\right) L_{4}^{r} \chi_{13} \chi_{4}+128 \bar{A}\left(\chi_{4}\right) L_{6}^{r} \chi_{13} \chi_{4}+16 / 27 \bar{A}\left(\chi_{4}\right) \bar{B}\left(\chi_{1}, \chi_{3}, 0\right) \chi_{13} \chi_{4}$

$+8 / 27 \bar{A}\left(\chi_{4}\right) \bar{B}\left(\chi_{1}, \chi_{3}, 0, k\right) \chi_{4}-2 \bar{A}\left(\chi_{4}, \varepsilon\right) \pi_{16} \chi_{13} \chi_{4}-\bar{B}\left(\chi_{p}, \chi_{p}, 0\right) \pi_{16}\left[1 / 24 R_{p}^{d} \chi_{q} \chi_{13}+1 / 8 R_{p}^{d} \chi_{13} \chi_{4}\right]$

$-4 / 3 \bar{B}\left(\chi_{p}, \chi_{p}, 0\right) L_{0}^{r} R_{p}^{d} \chi_{p} \chi_{13}-10 / 3 \bar{B}\left(\chi_{p}, \chi_{p}, 0\right) L_{3}^{r} R_{p}^{d} \chi_{p} \chi_{13}+8 \bar{B}\left(\chi_{p}, \chi_{p}, 0\right) L_{4}^{r} R_{q}^{p} \chi_{p} \chi_{13} \chi_{4}$

$+\bar{B}\left(\chi_{p}, \chi_{p}, 0\right) L_{5}^{r}\left[8 / 3 R_{q}^{p} \chi_{p}^{3}+4 / 3 R_{p}^{d} \chi_{1} \chi_{3}\right]-16 \bar{B}\left(\chi_{p}, \chi_{p}, 0\right) L_{6}^{r} R_{q}^{p} \chi_{p} \chi_{13} \chi_{4}$

- $\bar{B}\left(\chi_{p}, \chi_{p}, 0\right) L_{8}^{r}\left[16 / 3 R_{q}^{p} \chi_{p}^{3}+8 / 3 R_{p}^{d} \chi_{1} \chi_{3}\right]+\bar{B}\left(\chi_{p}, \chi_{p}, 0\right)^{2}\left[1 / 8 R_{q}^{p} R_{p}^{d} \chi_{p} \chi_{13}-1 / 72 R_{q}^{p} R_{p}^{d} \chi_{q} \chi_{13}\right]$

$+2 / 27 \bar{B}\left(\chi_{p}, \chi_{p}, 0\right) \bar{B}\left(\chi_{1}, \chi_{3}, 0\right) R_{p}^{q} R_{p}^{d} \chi_{p} \chi_{13}+1 / 27 \bar{B}\left(\chi_{p}, \chi_{p}, 0\right) \bar{B}\left(\chi_{1}, \chi_{3}, 0, k\right) R_{p}^{q} R_{p}^{d} \chi_{p}$

$+\bar{B}\left(\chi_{p}, \chi_{p}, 0, \varepsilon\right) \pi_{16}\left[1 / 4 R_{p}^{d} \chi_{p 4} \chi_{13}+5 / 36 R_{p}^{d} \chi_{13}^{2}\right]-1 / 36 \bar{B}\left(\chi_{1}, \chi_{1}, 0\right) \bar{B}\left(\chi_{3}, \chi_{3}, 0\right) R_{1}^{d} R_{3}^{d} \chi_{13}$

$+32 / 3 \bar{B}\left(\chi_{1}, \chi_{3}, 0\right) L_{7}^{r} R_{1}^{d} R_{3}^{d} \chi_{13}+32 / 9 \bar{B}\left(\chi_{1}, \chi_{3}, 0\right) L_{8}^{r} R_{1}^{d} R_{3}^{d} \chi_{13}+16 / 3 \bar{B}\left(\chi_{1}, \chi_{3}, 0, k\right) L_{7}^{r} R_{1}^{d} R_{3}^{d}$

$+16 / 9 \bar{B}\left(\chi_{1}, \chi_{3}, 0, k\right) L_{8}^{r} R_{1}^{d} R_{3}^{d}-H^{F}\left(1, \chi_{p}, \chi_{p}, \chi_{13}, \chi_{13}\right)\left[1 / 4 \chi_{p} \chi_{13}+5 / 12 \chi_{13}^{2}-1 / 6 R_{q}^{p} \chi_{13}^{2}\right.$

$\left.+1 / 18\left(R_{q}^{p}\right)^{2} \chi_{13}^{2}\right]+H^{F}\left(1, \chi_{p}, \chi_{14}, \chi_{34}, \chi_{13}\right)\left[1 / 4 \chi_{13}^{2}+3 / 4 R_{p}^{q} \chi_{p} \chi_{13}+1 / 4 R_{p}^{q} \chi_{q} \chi_{13}-1 / 4 R_{p}^{q} \chi_{13} \chi_{4}\right]$

$-H^{F}\left(1, \chi_{1}, \chi_{13}, \chi_{3}, \chi_{13}\right)\left[1 / 36 \chi_{13}^{2}-1 / 3 R_{3}^{1} R_{1}^{3} \chi_{13}^{2}\right]+1 / 4 H^{F}\left(1, \chi_{13}, \chi_{13}, \chi_{13}, \chi_{13}\right) \chi_{13}^{2}$

$+2 H^{F}\left(1, \chi_{14}, \chi_{34}, \chi_{4}, \chi_{13}\right) \chi_{13} \chi_{4}-H^{F}\left(2, \chi_{p}, \chi_{p}, \chi_{13}, \chi_{13}\right)\left[1 / 18 R_{q}^{p} R_{p}^{d} \chi_{13}^{2}-5 / 36 R_{p}^{d} \chi_{13}^{2}\right]$

$-H^{F}\left(2, \chi_{p}, \chi_{13}, \chi_{q}, \chi_{13}\right)\left[1 / 18 R_{p}^{q} R_{p}^{d} \chi_{13}^{2}-1 / 36 R_{p}^{d} \chi_{13}^{2}\right]-1 / 4 H^{F}\left(2, \chi_{p}, \chi_{14}, \chi_{34}, \chi_{13}\right) R_{p}^{d} \chi_{p 4} \chi_{13}$

$+5 / 72 H^{F}\left(5, \chi_{p}, \chi_{p}, \chi_{13}, \chi_{13}\right)\left(R_{p}^{d}\right)^{2} \chi_{13}^{2}+1 / 36 H^{F}\left(5, \chi_{1}, \chi_{3}, \chi_{13}, \chi_{13}\right) R_{1}^{d} R_{3}^{d} \chi_{13}^{2}$

$+H_{1}^{F}\left(1, \chi_{p}, \chi_{p}, \chi_{13}, \chi_{13}\right)\left[20 / 9 \chi_{13}^{2}-4 / 9 R_{q}^{p} R_{p}^{q} \chi_{13}^{2}\right]-2 H_{1}^{F}\left(1, \chi_{p}, \chi_{14}, \chi_{34}, \chi_{13}\right) R_{p}^{q} \chi_{13}^{2}$

$-2 H_{1}^{F}\left(1, \chi_{p 4}, \chi_{q 4}, \chi_{p}, \chi_{13}\right) \chi_{13}^{2}+H_{1}^{F}\left(1, \chi_{13}, \chi_{1}, \chi_{3}, \chi_{13}\right)\left[2 / 9 \chi_{13}^{2}-4 / 9 R_{3}^{1} R_{1}^{3} \chi_{13}^{2}\right]$

$+2 / 9 H_{1}^{F}\left(3, \chi_{13}, \chi_{p}, \chi_{p}, \chi_{13}\right)\left[R_{q}^{p} R_{p}^{d} \chi_{13}^{2}-R_{p}^{d} \chi_{13}^{2}\right]+2 / 9 H_{1}^{F}\left(3, \chi_{13}, \chi_{p}, \chi_{q}, \chi_{13}\right) R_{p}^{q} R_{p}^{d} \chi_{13}^{2}$

$-1 / 9 H_{1}^{F}\left(7, \chi_{13}, \chi_{p}, \chi_{p}, \chi_{13}\right)\left(R_{p}^{d}\right)^{2} \chi_{13}^{2}-3 / 4 H_{21}^{F}\left(1, \chi_{p}, \chi_{p}, \chi_{13}, \chi_{13}\right) \chi_{13}^{2}$

$+3 / 4 H_{21}^{F}\left(1, \chi_{p}, \chi_{14}, \chi_{34}, \chi_{13}\right) R_{p}^{q} \chi_{13}^{2}+3 / 4 H_{21}^{F}\left(1, \chi_{p 4}, \chi_{q 4}, \chi_{p}, \chi_{13}\right) R_{q}^{p} \chi_{13}^{2}$

$-3 / 4 H_{21}^{F}\left(1, \chi_{p 4}, \chi_{q 4}, \chi_{q}, \chi_{13}\right)\left[\chi_{13}^{2}-R_{p}^{q} \chi_{13}^{2}\right]+H_{21}^{F}\left(1, \chi_{13}, \chi_{p}, \chi_{p}, \chi_{13}\right)\left[5 / 12 \chi_{13}^{2}-1 / 6 R_{q}^{p} R_{p}^{q} \chi_{13}^{2}\right]$

$-H_{21}^{F}\left(1, \chi_{13}, \chi_{1}, \chi_{3}, \chi_{13}\right)\left[1 / 12 \chi_{13}^{2}-1 / 3 R_{3}^{1} R_{1}^{3} \chi_{13}^{2}\right]+3 / 4 H_{21}^{F}\left(1, \chi_{13}, \chi_{13}, \chi_{13}, \chi_{13}\right) \chi_{13}^{2}$

$+6 H_{21}^{F}\left(1, \chi_{4}, \chi_{14}, \chi_{34}, \chi_{13}\right) \chi_{13}^{2}-3 / 4 H_{21}^{F}\left(3, \chi_{p 4}, \chi_{p}, \chi_{q 4}, \chi_{13}\right) R_{p}^{d} \chi_{13}^{2}$

$-H_{21}^{F}\left(3, \chi_{13}, \chi_{p}, \chi_{p}, \chi_{13}\right)\left[1 / 6 R_{q}^{p} R_{p}^{d} \chi_{13}^{2}-1 / 12 R_{p}^{d} \chi_{13}^{2}\right]-H_{21}^{F}\left(3, \chi_{13}, \chi_{p}, \chi_{q}, \chi_{13}\right)\left[1 / 6 R_{p}^{q} R_{p}^{d} \chi_{13}^{2}\right.$

$\left.-1 / 12 R_{p}^{d} \chi_{13}^{2}\right]+1 / 24 H_{21}^{F}\left(7, \chi_{13}, \chi_{p}, \chi_{p}, \chi_{13}\right)\left(R_{p}^{d}\right)^{2} \chi_{13}^{2}+1 / 12 H_{21}^{F}\left(7, \chi_{13}, \chi_{1}, \chi_{3}, \chi_{13}\right) R_{1}^{d} R_{3}^{d} \chi_{13}^{2}$,

$$
\begin{aligned}
& \delta_{\text {loops }}^{(6) 22}=\pi_{16} L_{0}^{r}\left[-8 / 9 \chi_{\eta} \chi_{13} \chi_{4}+\chi_{1} \chi_{13} \chi_{3}-2 \chi_{13}^{3}+26 / 3 \bar{\chi}_{1} \chi_{13}^{2}+35 / 9 \bar{\chi}_{2} \chi_{13}\right]+4 \pi_{16} L_{1}^{r} \chi_{13}^{3} \\
& +\pi_{16} L_{2}^{r}\left[22 / 3 \chi_{\eta} \chi_{13} \chi_{4}+2 \chi_{13}^{3}+26 / 3 \bar{\chi}_{2} \chi_{13}\right]+\pi_{16} L_{3}^{r}\left[-8 / 9 \chi_{\eta} \chi_{13} \chi_{4}+7 / 6 \chi_{1} \chi_{13} \chi_{3}-11 / 3 \chi_{13}^{3}\right. \\
& \left.+17 / 3 \bar{\chi}_{1} \chi_{13}^{2}+43 / 18 \bar{\chi}_{2} \chi_{13}\right]+\pi_{16}^{2}\left[15 / 32 \chi_{\eta} \chi_{13} \chi_{4}+59 / 192 \chi_{1} \chi_{13} \chi_{3}-77 / 192 \chi_{13}^{3}+15 / 32 \bar{\chi}_{1} \chi_{13}^{2}\right. \\
& \left.+43 / 64 \bar{\chi}_{2} \chi_{13}\right]+384 L_{4}^{r} L_{5}^{r} \bar{\chi}_{1} \chi_{13}^{2}-1152 L_{4}^{r} L_{6}^{r} \bar{\chi}_{1}^{2} \chi_{13}-384 L_{4}^{r} L_{8}^{r} \bar{\chi}_{1} \chi_{13}^{2}+576 L_{4}^{r 2} \bar{\chi}_{1}^{2} \chi_{13} \\
& -384 L_{5}^{r} L_{6}^{r} \bar{\chi}_{1} \chi_{13}^{2}-128 L_{5}^{r} L_{8}^{r} \chi_{13}^{3}+64 L_{5}^{r 2} \chi_{13}^{3}+\bar{A}\left(\chi_{p}\right) \pi_{16}\left[1 / 4 \bar{\chi}_{1} R_{q \eta}^{p} \chi_{13}-1 / 8 \bar{\chi}_{1} R_{p}^{c} \chi_{13}\right. \\
& \left.+1 / 12 R_{q \eta}^{p} \chi_{13}^{2}-1 / 24 R_{p}^{c} \chi_{q} \chi_{13}\right]-\bar{A}\left(\chi_{p}\right) L_{0}^{r}\left[16 / 3 R_{q \eta}^{p} \chi_{p} \chi_{13}+4 / 3 R_{p}^{c} \chi_{p} \chi_{13}+4 / 3 R_{p}^{d} \chi_{13}\right] \\
& \text { - } \bar{A}\left(\chi_{p}\right) L_{3}^{r}\left[4 / 3 R_{q \eta}^{p} \chi_{p} \chi_{13}+10 / 3 R_{p}^{c} \chi_{p} \chi_{13}+10 / 3 R_{p}^{d} \chi_{13}\right]+16 \bar{A}\left(\chi_{p}\right) L_{4}^{r} \bar{\chi}_{1} R_{q \eta}^{p} \chi_{13} \\
& +\bar{A}\left(\chi_{p}\right) L_{5}^{r}\left[16 / 3 R_{q \eta}^{p} \chi_{p} \chi_{13}+8 / 3 R_{p}^{c} \chi_{p} \chi_{13}\right]-16 \bar{A}\left(\chi_{p}\right) L_{6}^{r} \bar{\chi}_{1} R_{q \eta}^{p} \chi_{13}+\bar{A}\left(\chi_{p}\right) L_{7}^{r}\left[32 / 3 R_{p}^{d} \chi_{p}\right. \\
& \left.+16 / 3 R_{p}^{d} \chi_{q}-8 / 3\left(R_{p}^{d}\right)^{2}\right]-\bar{A}\left(\chi_{p}\right) L_{8}^{r}\left[32 / 9 R_{q \eta}^{p} \chi_{1} \chi_{3}+64 / 9 R_{q \eta}^{p} \chi_{13}^{2}+16 / 3 R_{p}^{c} \chi_{p} \chi_{13}+8 / 9\left(R_{p}^{d}\right)^{2}\right] \\
& +\bar{A}\left(\chi_{p}\right)^{2}\left[1 / 8 \chi_{13}+1 / 18\left(R_{q \eta}^{p}\right)^{2} \chi_{13}+1 / 54 R_{q \eta}^{p} R_{p}^{c} \chi_{p}-1 / 54\left(R_{p}^{c}\right)^{2} \chi_{p}+1 / 72\left(R_{p}^{c}\right)^{2} \chi_{13}\right] \\
& +\bar{A}\left(\chi_{p}\right) \bar{A}\left(\chi_{p s}\right)\left[1 / 72 R_{q \eta}^{p} \chi_{p}+19 / 216 R_{q \eta}^{p} \chi_{q}-5 / 216 R_{s \eta}^{p} \chi_{p}-1 / 24 R_{s \eta}^{p} \chi_{q}+1 / 27 R_{p}^{c} \chi_{p}\right] \\
& +\bar{A}\left(\chi_{p}\right) \bar{A}\left(\chi_{q s}\right)\left[1 / 18 R_{q \eta}^{p} \chi_{q}-1 / 72 R_{s \eta}^{p} R_{q s p}^{z} \chi_{p}+1 / 216 R_{s \eta}^{p} R_{q s p}^{z} \chi_{q}+1 / 12 R_{p}^{c} \chi_{13}\right] \\
& +\bar{A}\left(\chi_{p}\right) \bar{A}\left(\chi_{\eta}\right)\left[1 / 162\left(R_{s \eta}^{p}\right)^{2} R_{s s}^{\eta} \chi_{s}+1 / 54 R_{q \eta}^{p} R_{p p}^{\eta} \chi_{p}-1 / 90 R_{q \eta}^{p} R_{q q}^{\eta} \chi_{q}+13 / 270 R_{q \eta}^{p} R_{13}^{\eta} \chi_{p}\right. \\
& +1 / 18 R_{q \eta}^{p} R_{13}^{\eta} \chi_{q}-1 / 135 R_{\eta \eta}^{p} R_{q q}^{\eta} \chi_{\eta}-1 / 54 R_{q q}^{\eta} R_{p}^{c} \chi_{p}+1 / 216 R_{p}^{c} R_{\eta 13}^{v} \chi_{p}+1 / 72 R_{p}^{c} R_{\eta 13}^{v} \chi_{q} \\
& \left.-1 / 36 R_{p}^{c} R_{\eta 13}^{v} \chi_{\eta}\right]+\bar{A}\left(\chi_{p}\right) \bar{A}\left(\chi_{13}\right)\left[1 / 18 \chi_{p}-1 / 36 \chi_{13}\right]-1 / 18 \bar{A}\left(\chi_{p}\right) \bar{A}\left(\chi_{4}\right)\left(R_{4 \eta}^{p}\right)^{2} \chi_{4}
\end{aligned}
$$


$-\bar{A}\left(\chi_{p}\right) \bar{A}\left(\chi_{46}\right)\left[2 / 27 R_{\eta \eta}^{p} \chi_{46}-1 / 9 R_{p p}^{\eta} \chi_{p}\right]+\bar{A}\left(\chi_{p}\right) \bar{B}\left(\chi_{p}, \chi_{p}, 0\right)\left[11 / 54 R_{q \eta}^{p} R_{p}^{c} \chi_{p} \chi_{13}-1 / 27 R_{q \eta}^{p} R_{p}^{c} \chi_{p}^{2}\right.$

$\left.-1 / 18 R_{q \eta}^{p} R_{p}^{c} \chi_{13}^{2}+1 / 54 R_{q \eta}^{p} R_{p}^{d} \chi_{p}\right]+\bar{A}\left(\chi_{p}\right) \bar{B}\left(\chi_{p}, \chi_{\eta}, 0\right)\left[2 / 27 R_{13}^{\eta} R_{p}^{c} \chi_{p}^{2}+1 / 27 R_{13}^{\eta} R_{p}^{c} \chi_{1} \chi_{3}\right]$

- $\bar{A}\left(\chi_{p}\right) \bar{B}\left(\chi_{q}, \chi_{q}, 0\right)\left[1 / 54 R_{q \eta}^{p} R_{q}^{d} \chi_{q}+1 / 36 R_{p}^{c} R_{q}^{d} \chi_{13}\right]+2 / 27 \bar{A}\left(\chi_{p}\right) \bar{B}\left(\chi_{1}, \chi_{3}, 0\right) R_{p \eta}^{q} R_{p}^{c} \chi_{p} \chi_{13}$

$+1 / 27 \bar{A}\left(\chi_{p}\right) \bar{B}\left(\chi_{1}, \chi_{3}, 0, k\right) R_{p \eta}^{q} R_{p}^{c} \chi_{p}+\bar{A}\left(\chi_{p}, \varepsilon\right) \pi_{16}\left[-1 / 4 \bar{\chi}_{1} R_{q \eta}^{p} \chi_{13}+1 / 8 \bar{\chi}_{1} R_{p}^{c} \chi_{13}+5 / 18 R_{q \eta}^{p} \chi_{p} \chi_{13}\right.$

$\left.+7 / 36 R_{p}^{c} \chi_{p} \chi_{13}+5 / 72 R_{p}^{c} \chi_{q} \chi_{13}-1 / 72 R_{p}^{d} \chi_{13}\right]-\bar{A}\left(\chi_{p s}\right) \pi_{16}\left[1 / 4 \chi_{p} \chi_{13}-1 / 8 \chi_{13} \chi_{s}-1 / 4 \chi_{13}^{2}\right.$

$\left.-3 / 8 \bar{\chi}_{1} \chi_{13}\right]+4 \bar{A}\left(\chi_{p s}\right) L_{0}^{r} \chi_{p s} \chi_{13}+10 \bar{A}\left(\chi_{p s}\right) L_{3}^{r} \chi_{p s} \chi_{13}-8 \bar{A}\left(\chi_{p s}\right) L_{5}^{r} \chi_{p s} \chi_{13}+16 \bar{A}\left(\chi_{p s}\right) L_{8}^{r} \chi_{p s} \chi_{13}$

$+\bar{A}\left(\chi_{p s}\right) \bar{A}\left(\chi_{\eta}\right)\left[1 / 27 R_{p p}^{\eta} \chi_{p}-5 / 216 R_{p s}^{\eta} \chi_{p}-1 / 24 R_{p s}^{\eta} \chi_{q}+1 / 12 R_{q q}^{\eta} \chi_{13}-5 / 72 R_{q s}^{\eta} \chi_{p}\right.$

- 19/216 $\left.R_{q s}^{\eta} R_{p s \eta}^{z} \chi_{q}+1 / 72 R_{13}^{\eta} \chi_{p}\right]-\bar{A}\left(\chi_{p s}\right) \bar{B}\left(\chi_{p}, \chi_{\eta}, 0\right)\left[2 / 9 R_{q s}^{\eta} \chi_{p} \chi_{13}+2 / 27 R_{q s}^{\eta} \chi_{p} \chi_{s}\right.$

$\left.+1 / 27 R_{q s}^{\eta} \chi_{q} \chi_{s}+1 / 27 R_{13}^{\eta} \chi_{p}^{2}-1 / 27 R_{13}^{\eta} \chi_{1} \chi_{3}\right]+1 / 12 \bar{A}\left(\chi_{p s}\right) \bar{B}\left(\chi_{q}, \chi_{q}, 0\right) R_{q}^{d} \chi_{13}$

$-\bar{A}\left(\chi_{p s}\right) \bar{B}\left(\chi_{1}, \chi_{3}, 0\right)\left[1 / 27 R_{p \eta}^{q} \chi_{p}^{2}+4 / 27 R_{s \eta}^{q} \chi_{p s} \chi_{13}+1 / 27 R_{s \eta}^{q} \chi_{1} \chi_{3}\right]$

$+\bar{A}\left(\chi_{p s}\right) \bar{B}\left(\chi_{1}, \chi_{3}, 0, k\right)\left[1 / 27 R_{p \eta}^{q} \chi_{q}-2 / 27 R_{s \eta}^{q} \chi_{p s}-2 / 27 R_{s \eta}^{q} \chi_{13}\right]-\bar{A}\left(\chi_{p s}, \varepsilon\right) \pi_{16}\left[3 / 8 \chi_{13} \chi_{s}\right.$

$\left.+5 / 12 \chi_{13}^{2}+3 / 8 \bar{\chi}_{1} \chi_{13}\right]-1 / 2 \bar{A}\left(\chi_{p 4}\right) \bar{A}\left(\chi_{q 6}\right) \chi_{13}-\bar{A}\left(\chi_{\eta}\right) \pi_{16}\left[1 / 8 \bar{\chi}_{1} R_{\eta 13}^{v} \chi_{13}-1 / 24 R_{\eta 13}^{v} \chi_{\eta} \chi_{13}\right.$

$\left.+1 / 12 R_{\eta 13}^{v} \chi_{13}^{2}\right]-\bar{A}\left(\chi_{\eta}\right) L_{0}^{r}\left[8 R_{13}^{\eta} \chi_{\eta} \chi_{13}+4 / 3 R_{\eta 13}^{v} \chi_{\eta} \chi_{13}\right]+16 \bar{A}\left(\chi_{\eta}\right) L_{1}^{r} \chi_{\eta} \chi_{13}+4 \bar{A}\left(\chi_{\eta}\right) L_{2}^{r} \chi_{\eta} \chi_{13}$

$-\bar{A}\left(\chi_{\eta}\right) L_{3}^{r}\left[8 R_{13}^{\eta} \chi_{\eta} \chi_{13}+10 / 3 R_{\eta 13}^{v} \chi_{\eta} \chi_{13}\right]-16 \bar{A}\left(\chi_{\eta}\right) L_{4}^{r}\left[\chi_{\eta} \chi_{13}-\bar{\chi}_{1} R_{13}^{\eta} \chi_{13}\right]$

$+\bar{A}\left(\chi_{\eta}\right) L_{5}^{r}\left[8 / 3 R_{p p}^{\eta} \chi_{\eta} \chi_{13}+16 / 3 R_{13}^{\eta} \chi_{13}^{2}\right]+16 \bar{A}\left(\chi_{\eta}\right) L_{6}^{r}\left[\chi_{\eta} \chi_{13}-\bar{\chi}_{1} R_{13}^{\eta} \chi_{13}\right]$

$+\bar{A}\left(\chi_{\eta}\right) L_{7}^{r}\left[64 / 3 R_{13}^{\eta} \chi_{\eta} \chi_{13}-64 / 3 R_{13}^{\eta} \chi_{13}^{2}-4 / 3\left(R_{\eta 4}^{z}\right)^{2} R_{\eta 13}^{v}+16 / 3 R_{\eta 46 p}^{z} \chi_{q}\right]$

$-\bar{A}\left(\chi_{\eta}\right) L_{8}^{r}\left[64 / 9 R_{13}^{\eta} \chi_{1} \chi_{3}+128 / 9 R_{13}^{\eta} \chi_{13}^{2}+16 / 9 R_{\eta 13}^{v} \chi_{\eta}^{2}+8 / 3 R_{\eta 13}^{v} \chi_{1} \chi_{3}+8 / 27 R_{\eta 13}^{v} \chi_{4}^{2}\right.$

$\left.+16 / 27 R_{\eta 13}^{v} \chi_{6}^{2}\right]+\bar{A}\left(\chi_{\eta}\right)^{2}\left[1 / 54 \bar{\chi}_{1} R_{\eta 13}^{v}+1 / 18\left(R_{13}^{\eta}\right)^{2} \chi_{13}-1 / 54 R_{13}^{\eta} R_{\eta 13}^{v} \chi_{\eta}+1 / 54 R_{13}^{\eta} R_{\eta 13}^{v} \chi_{13}\right.$

$\left.-5 / 108 R_{\eta 13}^{v} \chi_{\eta}-1 / 54\left(R_{\eta 13}^{v}\right)^{2} \chi_{\eta}+1 / 72\left(R_{\eta 13}^{v}\right)^{2} \chi_{13}\right]-1 / 36 \bar{A}\left(\chi_{\eta}\right) \bar{A}\left(\chi_{4}\right) R_{\eta 13}^{v} \chi_{4}$

$+\bar{A}\left(\chi_{\eta}\right) \bar{A}\left(\chi_{46}\right)\left[1 / 9 R_{13}^{\eta} \chi_{13}+2 / 27 R_{\eta 13}^{v} \chi_{46}\right]+\bar{A}\left(\chi_{\eta}\right) \bar{B}\left(\chi_{p}, \chi_{p}, 0\right)\left[1 / 9 R_{q \eta}^{p} R_{p p}^{\eta} \chi_{p}^{2}-1 / 27 R_{p p}^{\eta} R_{p}^{d} \chi_{p}\right.$

$\left.-1 / 54 R_{q q}^{\eta} R_{p}^{d} \chi_{p}+1 / 216 R_{p}^{d} R_{\eta 13}^{v} \chi_{p}+1 / 72 R_{p}^{d} R_{\eta 13}^{v} \chi_{q}-1 / 36 R_{p}^{d} R_{\eta 13}^{v} \chi_{\eta}\right]$

- $\bar{A}\left(\chi_{\eta}\right) \bar{B}\left(\chi_{p}, \chi_{\eta}, 0\right)\left[2 / 27 R_{\eta \eta}^{p} R_{q 4}^{\eta} \chi_{p} \chi_{\eta}+1 / 27 R_{\eta \eta}^{p} R_{q 4}^{\eta} \chi_{q} \chi_{\eta}-2 / 27 R_{\eta \eta}^{p} R_{13}^{\eta} \chi_{p}^{2}-1 / 27 R_{\eta \eta}^{p} R_{13}^{\eta} \chi_{1} \chi_{3}\right]$

$+\bar{A}\left(\chi_{\eta}\right) \bar{B}\left(\chi_{\eta}, \chi_{\eta}, 0\right)\left[1 / 9 \bar{\chi}_{1} R_{13}^{\eta} \chi_{13}-5 / 18 R_{13}^{\eta} \chi_{\eta} \chi_{13}\right]+2 / 27 \bar{A}\left(\chi_{\eta}\right) \bar{B}\left(\chi_{1}, \chi_{3}, 0\right) R_{\eta \eta}^{1} R_{\eta \eta}^{3} \chi_{\eta} \chi_{13}$

$+1 / 27 \bar{A}\left(\chi_{\eta}\right) \bar{B}\left(\chi_{1}, \chi_{3}, 0, k\right) R_{\eta \eta}^{1} R_{\eta \eta}^{3} \chi_{\eta}+\bar{A}\left(\chi_{\eta}, \varepsilon\right) \pi_{16}\left[-1 / 4 \chi_{\eta} \chi_{13}+1 / 8 \bar{\chi}_{1} R_{\eta 13}^{v} \chi_{13}+1 / 4 R_{13}^{\eta} \chi_{\eta} \chi_{13}\right.$

$\left.+5 / 9 R_{13}^{\eta} \chi_{13}^{2}+1 / 8 R_{\eta 13}^{v} \chi_{\eta} \chi_{13}+5 / 36 R_{\eta 13}^{v} \chi_{13}^{2}\right]-\bar{A}\left(\chi_{1}\right) \bar{A}\left(\chi_{3}\right)\left[1 / 54 R_{q \eta}^{p} R_{q}^{c} \chi_{q}-1 / 9 R_{3 \eta}^{1} R_{1 \eta}^{3} \chi_{13}\right.$

$\left.+1 / 36 R_{1}^{c} R_{3}^{c} \chi_{13}\right]+8 \bar{A}\left(\chi_{13}\right) L_{1}^{r} \chi_{13}^{2}+20 \bar{A}\left(\chi_{13}\right) L_{2}^{r} \chi_{13}^{2}+32 \bar{A}\left(\chi_{13}\right) L_{6}^{r} \chi_{13}^{2}-1 / 4 \bar{A}\left(\chi_{13}\right)^{2} \chi_{13}$

$+\bar{A}\left(\chi_{13}\right) \bar{B}\left(\chi_{1}, \chi_{3}, 0\right)\left[1 / 9 \chi_{1} \chi_{3}+4 / 9 \chi_{13}^{2}\right]+4 / 9 \bar{A}\left(\chi_{13}\right) \bar{B}\left(\chi_{1}, \chi_{3}, 0, k\right) \chi_{13}-31 / 18 \bar{A}\left(\chi_{13}, \varepsilon\right) \pi_{16} \chi_{13}^{2}$

$-\bar{A}\left(\chi_{14}\right) \bar{A}\left(\chi_{34}\right) \chi_{13}-1 / 4 \bar{A}\left(\chi_{16}\right) \bar{A}\left(\chi_{36}\right) \chi_{13}+48 \bar{A}\left(\chi_{4}\right) L_{1}^{r} \chi_{13} \chi_{4}+12 \bar{A}\left(\chi_{4}\right) L_{2}^{r} \chi_{13} \chi_{4}$

$-48 \bar{A}\left(\chi_{4}\right) L_{4}^{r} \chi_{13} \chi_{4}+48 \bar{A}\left(\chi_{4}\right) L_{6}^{r} \chi_{13} \chi_{4}+\bar{A}\left(\chi_{4}\right) \bar{B}\left(\chi_{p}, \chi_{\eta}, 0\right)\left[2 / 9 R_{4 \eta}^{p} R_{q 4}^{\eta} \chi_{p} \chi_{4}+1 / 9 R_{4 \eta}^{p} R_{q 4}^{\eta} \chi_{q} \chi_{4}\right]$

- $1 / 6 \bar{A}\left(\chi_{4}\right) \bar{B}\left(\chi_{\eta}, \chi_{\eta}, 0\right) R_{13}^{\eta} \chi_{13} \chi_{4}+2 / 9 \bar{A}\left(\chi_{4}\right) \bar{B}\left(\chi_{1}, \chi_{3}, 0\right) R_{4 \eta}^{1} R_{4 \eta}^{3} \chi_{13} \chi_{4}$

$+1 / 9 \bar{A}\left(\chi_{4}\right) \bar{B}\left(\chi_{1}, \chi_{3}, 0, k\right) R_{4 \eta}^{1} R_{4 \eta}^{3} \chi_{4}-3 / 4 \bar{A}\left(\chi_{4}, \varepsilon\right) \pi_{16} \chi_{13} \chi_{4}+64 \bar{A}\left(\chi_{46}\right) L_{1}^{r} \chi_{13} \chi_{46}$

$+16 \bar{A}\left(\chi_{46}\right) L_{2}^{r} \chi_{13} \chi_{46}-64 \bar{A}\left(\chi_{46}\right) L_{4}^{r} \chi_{13} \chi_{46}+64 \bar{A}\left(\chi_{46}\right) L_{6}^{r} \chi_{13} \chi_{46}$

$+\bar{A}\left(\chi_{46}\right) \bar{B}\left(\chi_{p}, \chi_{\eta}, 0\right)\left[2 / 81 R_{p p 46}^{u \eta} \chi_{p} \chi_{\eta}-2 / 81 R_{p q 46}^{u \eta} \chi_{p} \chi_{\eta}-2 / 81 R_{p q 46}^{u \eta} \chi_{1} \chi_{3}+4 / 81 R_{p q 46}^{w \eta} \chi_{p}\right.$

$\left.+2 / 81 R_{p q 46}^{w \eta} \chi_{q}\right]-2 / 81 \bar{A}\left(\chi_{46}\right) \bar{B}\left(\chi_{p}, \chi_{\eta}, 0, k\right)\left[R_{p p 46}^{u \eta} \chi_{p}+R_{p q 46}^{u \eta} \chi_{p}\right]$

$+4 / 9 \bar{A}\left(\chi_{46}\right) \bar{B}\left(\chi_{\eta}, \chi_{\eta}, 0\right) R_{13}^{\eta} \chi_{13} \chi_{46}-\bar{A}\left(\chi_{46}\right) \bar{B}\left(\chi_{1}, \chi_{3}, 0\right)\left[2 / 81 R_{1346}^{u} \chi_{1} \chi_{3}-4 / 81 R_{1346}^{w} \chi_{13}\right]$

- $\bar{A}\left(\chi_{46}\right) \bar{B}\left(\chi_{1}, \chi_{3}, 0, k\right)\left[4 / 81 R_{1346}^{u} \chi_{13}-2 / 81 R_{1346}^{w}\right]-\bar{A}\left(\chi_{46}, \varepsilon\right) \pi_{16} \chi_{13} \chi_{46}$

$-\bar{B}\left(\chi_{p}, \chi_{p}, 0\right) \pi_{16}\left[1 / 8 \bar{\chi}_{1} R_{p}^{d} \chi_{13}+1 / 24 R_{p}^{d} \chi_{q} \chi_{13}\right]-4 / 3 \bar{B}\left(\chi_{p}, \chi_{p}, 0\right) L_{0}^{r} R_{p}^{d} \chi_{p} \chi_{13}$

- $10 / 3 \bar{B}\left(\chi_{p}, \chi_{p}, 0\right) L_{3}^{r} R_{p}^{d} \chi_{p} \chi_{13}+8 \bar{B}\left(\chi_{p}, \chi_{p}, 0\right) L_{4}^{r} \bar{\chi}_{1} R_{q \eta}^{p} \chi_{p} \chi_{13}$

$+\bar{B}\left(\chi_{p}, \chi_{p}, 0\right) L_{5}^{r}\left[16 / 3 R_{q \eta}^{p} \chi_{p}^{2} \chi_{13}-8 / 3 R_{q \eta}^{p} \chi_{1} \chi_{13} \chi_{3}\right]-16 \bar{B}\left(\chi_{p}, \chi_{p}, 0\right) L_{6}^{r} \bar{\chi}_{1} R_{q \eta}^{p} \chi_{p} \chi_{13}$

- $\bar{B}\left(\chi_{p}, \chi_{p}, 0\right) L_{8}^{r}\left[32 / 3 R_{q \eta}^{p} \chi_{p}^{2} \chi_{13}-16 / 3 R_{q \eta}^{p} \chi_{1} \chi_{13} \chi_{3}\right]+\bar{B}\left(\chi_{p}, \chi_{p}, 0\right)^{2}\left[1 / 8 R_{q \eta}^{p} R_{p}^{d} \chi_{p} \chi_{13}\right.$

$\left.-1 / 72 R_{q \eta}^{p} R_{p}^{d} \chi_{q} \chi_{13}\right]+\bar{B}\left(\chi_{p}, \chi_{p}, 0\right) \bar{B}\left(\chi_{p}, \chi_{\eta}, 0\right)\left[2 / 27 R_{13}^{\eta} R_{p}^{d} \chi_{p}^{2}+1 / 27 R_{13}^{\eta} R_{p}^{d} \chi_{1} \chi_{3}\right]$ 
$+2 / 27 \bar{B}\left(\chi_{p}, \chi_{p}, 0\right) \bar{B}\left(\chi_{1}, \chi_{3}, 0\right) R_{p \eta}^{q} R_{p}^{d} \chi_{p} \chi_{13}+1 / 27 \bar{B}\left(\chi_{p}, \chi_{p}, 0\right) \bar{B}\left(\chi_{1}, \chi_{3}, 0, k\right) R_{p \eta}^{q} R_{p}^{d} \chi_{p}$

$+\bar{B}\left(\chi_{p}, \chi_{p}, 0, \varepsilon\right) \pi_{16}\left[1 / 8 \bar{\chi}_{1} R_{p}^{d} \chi_{13}+7 / 36 R_{p}^{d} \chi_{p} \chi_{13}+5 / 72 R_{p}^{d} \chi_{q} \chi_{13}\right]$

$+\bar{B}\left(\chi_{p}, \chi_{\eta}, 0\right) L_{7}^{r}\left[32 / 3 R_{p}^{d} R_{\eta 46 q}^{z} \chi_{p}+16 / 3 R_{p}^{d} R_{\eta 46 q}^{z} \chi_{q}\right]+\bar{B}\left(\chi_{p}, \chi_{\eta}, 0\right) L_{8}^{r}\left[32 / 9 R_{p}^{d} R_{\eta 46 q}^{z} \chi_{p}\right.$

$\left.+16 / 9 R_{p}^{d} R_{\eta 46 q}^{z} \chi_{q}\right]+8 \bar{B}\left(\chi_{\eta}, \chi_{\eta}, 0\right) L_{4}^{r} \bar{\chi}_{1} R_{13}^{\eta} \chi_{\eta} \chi_{13}+8 / 3 \bar{B}\left(\chi_{\eta}, \chi_{\eta}, 0\right) L_{5}^{r} R_{13}^{\eta} \chi_{\eta}^{2} \chi_{13}$

- $16 \bar{B}\left(\chi_{\eta}, \chi_{\eta}, 0\right) L_{6}^{r} \bar{\chi}_{1} R_{13}^{\eta} \chi_{\eta} \chi_{13}-8 \bar{B}\left(\chi_{\eta}, \chi_{\eta}, 0\right) L_{7}^{r} R_{13}^{\eta}\left(R_{\eta 4}^{z}\right)^{2} \chi_{13}-\bar{B}\left(\chi_{\eta}, \chi_{\eta}, 0\right) L_{8}^{r}\left[16 / 9 R_{13}^{\eta} \chi_{13} \chi_{4}^{2}\right.$

$\left.+32 / 9 R_{13}^{\eta} \chi_{13} \chi_{6}^{2}\right]-1 / 36 \bar{B}\left(\chi_{1}, \chi_{1}, 0\right) \bar{B}\left(\chi_{3}, \chi_{3}, 0\right) R_{1}^{d} R_{3}^{d} \chi_{13}+32 / 3 \bar{B}\left(\chi_{1}, \chi_{3}, 0\right) L_{7}^{r} R_{1}^{d} R_{3}^{d} \chi_{13}$

$+32 / 9 \bar{B}\left(\chi_{1}, \chi_{3}, 0\right) L_{8}^{r} R_{1}^{d} R_{3}^{d} \chi_{13}+16 / 3 \bar{B}\left(\chi_{1}, \chi_{3}, 0, k\right) L_{7}^{r} R_{1}^{d} R_{3}^{d}+16 / 9 \bar{B}\left(\chi_{1}, \chi_{3}, 0, k\right) L_{8}^{r} R_{1}^{d} R_{3}^{d}$

- $H^{F}\left(1, \chi_{p}, \chi_{p}, \chi_{13}, \chi_{13}\right)\left[1 / 4 \chi_{p} \chi_{13}+3 / 8 \chi_{13}^{2}+1 / 18\left(R_{q \eta}^{p}\right)^{2} \chi_{13}^{2}-1 / 6 R_{q \eta}^{p} R_{p}^{c} \chi_{13}^{2}+1 / 24\left(R_{p}^{c}\right)^{2} \chi_{13}^{2}\right]$

$+H^{F}\left(1, \chi_{p}, \chi_{1 s}, \chi_{3 s}, \chi_{13}\right)\left[-5 / 12 R_{q \eta}^{p} \chi_{q} \chi_{13}+1 / 6 R_{q \eta}^{p} \chi_{p s} \chi_{13}+1 / 8 R_{s \eta}^{p} \chi_{q} \chi_{13}+7 / 24 R_{s \eta}^{p} \chi_{13} \chi_{s}\right.$

$\left.-1 / 12 R_{p}^{c} \chi_{p s} \chi_{13}\right]+1 / 2 H^{F}\left(1, \chi_{p 4}, \chi_{q 6}, \chi_{46}, \chi_{13}\right) \chi_{13} \chi_{46}+H^{F}\left(1, \chi_{\eta}, \chi_{p}, \chi_{13}, \chi_{13}\right)\left[2 / 9 R_{q \eta}^{p} R_{13}^{\eta} \chi_{13}^{2}\right.$

$\left.-1 / 18 R_{q \eta}^{p} R_{\eta 13}^{v} \chi_{13}^{2}+1 / 9 R_{p p}^{\eta} R_{p}^{c} \chi_{13}^{2}+1 / 36 R_{p}^{c} R_{\eta 13}^{v} \chi_{13}^{2}\right]+H^{F}\left(1, \chi_{\eta}, \chi_{\eta}, \chi_{13}, \chi_{13}\right)\left[2 / 9\left(R_{13}^{\eta}\right)^{2} \chi_{13}^{2}\right.$

$\left.+2 / 9 R_{13}^{\eta} R_{\eta 13}^{v} \chi_{13}^{2}+5 / 72\left(R_{\eta 13}^{v}\right)^{2} \chi_{13}^{2}\right]-H^{F}\left(1, \chi_{\eta}, \chi_{1 s}, \chi_{3 s}, \chi_{13}\right)\left[1 / 8 R_{p s}^{\eta} \chi_{p} \chi_{13}+5 / 24 R_{p s}^{\eta} \chi_{q} \chi_{13}\right.$

$\left.-1 / 3 R_{13}^{\eta} \chi_{13}^{2}+1 / 24 R_{\eta p s}^{v} \chi_{\eta} \chi_{13}+1 / 24 R_{\eta 13}^{v} \chi_{13} \chi_{s}\right]-H^{F}\left(1, \chi_{1}, \chi_{13}, \chi_{3}, \chi_{13}\right)\left[1 / 18 R_{q \eta}^{p} R_{q}^{c} \chi_{13}^{2}\right.$

$\left.-1 / 3 R_{3 \eta}^{1} R_{1 \eta}^{3} \chi_{13}^{2}-1 / 36 R_{1}^{c} R_{3}^{c} \chi_{13}^{2}\right]+1 / 4 H^{F}\left(1, \chi_{13}, \chi_{13}, \chi_{13}, \chi_{13}\right) \chi_{13}^{2}$

$+3 / 4 H^{F}\left(1, \chi_{14}, \chi_{34}, \chi_{4}, \chi_{13}\right) \chi_{13} \chi_{4}-H^{F}\left(2, \chi_{p}, \chi_{p}, \chi_{13}, \chi_{13}\right)\left[1 / 18 R_{q \eta}^{p} R_{p}^{d} \chi_{13}^{2}-5 / 36 R_{p}^{c} R_{p}^{d} \chi_{13}^{2}\right]$

$+H^{F}\left(2, \chi_{p}, \chi_{\eta}, \chi_{13}, \chi_{13}\right)\left[1 / 9 R_{p p}^{\eta} R_{p}^{d} \chi_{13}^{2}+1 / 36 R_{p}^{d} R_{\eta 13}^{v} \chi_{13}^{2}\right]-H^{F}\left(2, \chi_{p}, \chi_{13}, \chi_{q}, \chi_{13}\right)\left[1 / 18 R_{p \eta}^{q} R_{p}^{d} \chi_{13}^{2}\right.$

$\left.-1 / 36 R_{q}^{c} R_{p}^{d} \chi_{13}^{2}\right]-1 / 12 H^{F}\left(2, \chi_{p}, \chi_{1 s}, \chi_{3 s}, \chi_{13}\right) R_{p}^{d} \chi_{p s} \chi_{13}+5 / 72 H^{F}\left(5, \chi_{p}, \chi_{p}, \chi_{13}, \chi_{13}\right)\left(R_{p}^{d}\right)^{2} \chi_{13}^{2}$

$+1 / 36 H^{F}\left(5, \chi_{1}, \chi_{3}, \chi_{13}, \chi_{13}\right) R_{1}^{d} R_{3}^{d} \chi_{13}^{2}+H_{1}^{F}\left(1, \chi_{p}, \chi_{p}, \chi_{13}, \chi_{13}\right)\left[2 \chi_{13}^{2}+4 / 9\left(R_{q \eta}^{p}\right)^{2} \chi_{13}^{2}\right.$

$\left.-4 / 9 R_{q \eta}^{p} R_{p}^{c} \chi_{13}^{2}+2 / 9\left(R_{p}^{c}\right)^{2} \chi_{13}^{2}\right]+2 / 3 H_{1}^{F}\left(1, \chi_{p}, \chi_{1 s}, \chi_{3 s}, \chi_{13}\right) R_{q \eta}^{p} R_{s q p}^{z} \chi_{13}^{2}$

$-2 / 3 H_{1}^{F}\left(1, \chi_{p s}, \chi_{q s}, \chi_{p}, \chi_{13}\right) R_{s \eta}^{p} \chi_{13}^{2}-2 / 3 H_{1}^{F}\left(1, \chi_{p s}, \chi_{q s}, \chi_{\eta}, \chi_{13}\right) R_{13}^{\eta} R_{s p \eta}^{z} \chi_{13}^{2}$

$+2 / 9 H_{1}^{F}\left(1, \chi_{13}, \chi_{p}, \chi_{\eta}, \chi_{13}\right)\left[R_{q \eta}^{p} R_{\eta 13}^{v} \chi_{13}^{2}-R_{p p}^{\eta} R_{q p \eta}^{z} R_{p}^{c} \chi_{13}^{2}\right]-H_{1}^{F}\left(1, \chi_{13}, \chi_{\eta}, \chi_{\eta}, \chi_{13}\right)\left[2 / 9 R_{13}^{\eta} R_{\eta 13}^{v} \chi_{13}^{2}\right.$

$\left.+1 / 9\left(R_{\eta 13}^{v}\right)^{2} \chi_{13}^{2}\right]+H_{1}^{F}\left(1, \chi_{13}, \chi_{1}, \chi_{3}, \chi_{13}\right)\left[2 / 9 R_{q \eta}^{p} R_{q}^{c} \chi_{13}^{2}-4 / 9 R_{3 \eta}^{1} R_{1 \eta}^{3} \chi_{13}^{2}\right]$

$+2 / 9 H_{1}^{F}\left(3, \chi_{13}, \chi_{p}, \chi_{p}, \chi_{13}\right)\left[R_{q \eta}^{p} R_{p}^{d} \chi_{13}^{2}-R_{p}^{c} R_{p}^{d} \chi_{13}^{2}\right]+2 / 9 H_{1}^{F}\left(3, \chi_{13}, \chi_{p}, \chi_{q}, \chi_{13}\right) R_{p \eta}^{q} R_{p}^{d} \chi_{13}^{2}$

$+2 / 9 H_{1}^{F}\left(3, \chi_{13}, \chi_{p}, \chi_{\eta}, \chi_{13}\right) R_{13}^{\eta} R_{p q \eta}^{z} R_{p}^{d} \chi_{13}^{2}-1 / 9 H_{1}^{F}\left(7, \chi_{13}, \chi_{p}, \chi_{p}, \chi_{13}\right)\left(R_{p}^{d}\right)^{2} \chi_{13}^{2}$

$-3 / 4 H_{21}^{F}\left(1, \chi_{p}, \chi_{p}, \chi_{13}, \chi_{13}\right) \chi_{13}^{2}-1 / 4 H_{21}^{F}\left(1, \chi_{p}, \chi_{1 s}, \chi_{3 s}, \chi_{13}\right) R_{q \eta}^{p} R_{s q p}^{z} \chi_{13}^{2}$

$+1 / 4 H_{21}^{F}\left(1, \chi_{p s}, \chi_{q s}, \chi_{p}, \chi_{13}\right)\left[R_{q \eta}^{p} \chi_{13}^{2}+R_{s \eta}^{p} \chi_{13}^{2}-R_{p}^{c} \chi_{13}^{2}\right]+1 / 4 H_{21}^{F}\left(1, \chi_{p s}, \chi_{q s}, \chi_{q}, \chi_{13}\right) R_{p \eta}^{q} R_{s p q}^{z} \chi_{13}^{2}$

$+1 / 4 H_{21}^{F}\left(1, \chi_{p s}, \chi_{q s}, \chi_{\eta}, \chi_{13}\right) R_{13}^{\eta} R_{p q \eta}^{z} R_{s p \eta}^{z} \chi_{13}^{2}-1 / 4 H_{21}^{F}\left(1, \chi_{\eta}, \chi_{1 s}, \chi_{3 s}, \chi_{13}\right) R_{13}^{\eta} R_{s 1 \eta}^{z} R_{s 3 \eta}^{z} \chi_{13}^{2}$

$+H_{21}^{F}\left(1, \chi_{13}, \chi_{p}, \chi_{p}, \chi_{13}\right)\left[3 / 8 \chi_{13}^{2}+1 / 6\left(R_{q \eta}^{p}\right)^{2} \chi_{13}^{2}-1 / 6 R_{q \eta}^{p} R_{p}^{c} \chi_{13}^{2}+1 / 24\left(R_{p}^{c}\right)^{2} \chi_{13}^{2}\right]$

- $H_{21}^{F}\left(1, \chi_{13}, \chi_{p}, \chi_{\eta}, \chi_{13}\right)\left[1 / 6 R_{q \eta}^{p} R_{\eta 13}^{v} \chi_{13}^{2}-1 / 12 R_{p}^{c} R_{\eta 13}^{v} \chi_{13}^{2}\right]+1 / 24 H_{21}^{F}\left(1, \chi_{13}, \chi_{\eta}, \chi_{\eta}, \chi_{13}\right)\left(R_{\eta 13}^{v}\right)^{2} \chi_{13}^{2}$

$-H_{21}^{F}\left(1, \chi_{13}, \chi_{1}, \chi_{3}, \chi_{13}\right)\left[1 / 6 R_{q \eta}^{p} R_{q}^{c} \chi_{13}^{2}-1 / 3 R_{3 \eta}^{1} R_{1 \eta}^{3} \chi_{13}^{2}-1 / 12 R_{1}^{c} R_{3}^{c} \chi_{13}^{2}\right]$

$+3 / 4 H_{21}^{F}\left(1, \chi_{13}, \chi_{13}, \chi_{13}, \chi_{13}\right) \chi_{13}^{2}+9 / 4 H_{21}^{F}\left(1, \chi_{4}, \chi_{14}, \chi_{34}, \chi_{13}\right) \chi_{13}^{2}+3 / 2 H_{21}^{F}\left(1, \chi_{46}, \chi_{p 4}, \chi_{q 6}, \chi_{13}\right) \chi_{13}^{2}$

$-1 / 4 H_{21}^{F}\left(3, \chi_{p s}, \chi_{p}, \chi_{q s}, \chi_{13}\right) R_{p}^{d} \chi_{13}^{2}-H_{21}^{F}\left(3, \chi_{13}, \chi_{p}, \chi_{p}, \chi_{13}\right)\left[1 / 6 R_{q \eta}^{p} R_{p}^{d} \chi_{13}^{2}-1 / 12 R_{p}^{c} R_{p}^{d} \chi_{13}^{2}\right]$

$-H_{21}^{F}\left(3, \chi_{13}, \chi_{p}, \chi_{q}, \chi_{13}\right)\left[1 / 6 R_{p \eta}^{q} R_{p}^{d} \chi_{13}^{2}-1 / 12 R_{q}^{c} R_{p}^{d} \chi_{13}^{2}\right]+1 / 12 H_{21}^{F}\left(3, \chi_{13}, \chi_{p}, \chi_{\eta}, \chi_{13}\right) R_{p}^{d} R_{\eta 13}^{v} \chi_{13}^{2}$

$+1 / 24 H_{21}^{F}\left(7, \chi_{13}, \chi_{p}, \chi_{p}, \chi_{13}\right)\left(R_{p}^{d}\right)^{2} \chi_{13}^{2}+1 / 12 H_{21}^{F}\left(7, \chi_{13}, \chi_{1}, \chi_{3}, \chi_{13}\right) R_{1}^{d} R_{3}^{d} \chi_{13}^{2}$,

$$
\begin{aligned}
\delta_{\text {loops }}^{(6) 23}= & \pi_{16} L_{0}^{r}\left[-8 / 9 \chi_{\pi} \chi_{\eta} \chi_{13}+\chi_{1} \chi_{13} \chi_{3}-2 \chi_{13}^{3}+26 / 3 \bar{\chi}_{1} \chi_{13}^{2}+35 / 9 \bar{\chi}_{2} \chi_{13}\right]+4 \pi_{16} L_{1}^{r} \chi_{13}^{3} \\
& +\pi_{16} L_{2}^{r}\left[22 / 3 \chi_{\pi} \chi_{\eta} \chi_{13}+2 \chi_{13}^{3}+26 / 3 \bar{\chi}_{2} \chi_{13}\right]+\pi_{16} L_{3}^{r}\left[-8 / 9 \chi_{\pi} \chi_{\eta} \chi_{13}+7 / 6 \chi_{1} \chi_{13} \chi_{3}-11 / 3 \chi_{13}^{3}\right. \\
& \left.+17 / 3 \bar{\chi}_{1} \chi_{13}^{2}+43 / 18 \bar{\chi}_{2} \chi_{13}\right]+\pi_{16}^{2}\left[15 / 32 \chi_{\pi} \chi_{\eta} \chi_{13}+59 / 192 \chi_{1} \chi_{13} \chi_{3}-77 / 192 \chi_{13}^{3}+15 / 32 \bar{\chi}_{1} \chi_{13}^{2}\right. \\
& \left.+43 / 64 \bar{\chi}_{2} \chi_{13}\right]+384 L_{4}^{r} L_{5}^{r} \bar{\chi}_{1} \chi_{13}^{2}-1152 L_{4}^{r} L_{6}^{r} \bar{\chi}_{1}^{2} \chi_{13}-384 L_{4}^{r} L_{8}^{r} \bar{\chi}_{1} \chi_{13}^{2}+576 L_{4}^{r 2} \bar{\chi}_{1}^{2} \chi_{13} \\
& -384 L_{5}^{r} L_{6}^{r} \bar{\chi}_{1} \chi_{13}^{2}-128 L_{5}^{r} L_{8}^{r} \chi_{13}^{3}+64 L_{5}^{r 2} \chi_{13}^{3}+\bar{A}\left(\chi_{p}\right) \pi_{16}\left[1 / 4 \bar{\chi}_{1} R_{q \pi \eta}^{p} \chi_{13}-1 / 8 \bar{\chi}_{1} R_{p}^{c} \chi_{13}\right.
\end{aligned}
$$


$\left.+1 / 12 R_{q \pi \eta}^{p} \chi_{13}^{2}-1 / 24 R_{p}^{c} \chi_{q} \chi_{13}\right]-\bar{A}\left(\chi_{p}\right) L_{0}^{r}\left[16 / 3 R_{q \pi \eta}^{p} \chi_{p} \chi_{13}+4 / 3 R_{p}^{c} \chi_{p} \chi_{13}+4 / 3 R_{p}^{d} \chi_{13}\right]$

$-\bar{A}\left(\chi_{p}\right) L_{3}^{r}\left[4 / 3 R_{q \pi \eta}^{p} \chi_{p} \chi_{13}+10 / 3 R_{p}^{c} \chi_{p} \chi_{13}+10 / 3 R_{p}^{d} \chi_{13}\right]+16 \bar{A}\left(\chi_{p}\right) L_{4}^{r} \bar{\chi}_{1} R_{q \pi \eta}^{p} \chi_{13}$

$+\bar{A}\left(\chi_{p}\right) L_{5}^{r}\left[16 / 3 R_{q \pi \eta}^{p} \chi_{p} \chi_{13}+8 / 3 R_{p}^{c} \chi_{p} \chi_{13}\right]-16 \bar{A}\left(\chi_{p}\right) L_{6}^{r} \bar{\chi}_{1} R_{q \pi \eta}^{p} \chi_{13}+\bar{A}\left(\chi_{p}\right) L_{7}^{r}\left[32 / 3 R_{p}^{d} \chi_{p}\right.$

$\left.+16 / 3 R_{p}^{d} \chi_{q}-8 / 3\left(R_{p}^{d}\right)^{2}\right]-\bar{A}\left(\chi_{p}\right) L_{8}^{r}\left[32 / 9 R_{q \pi \eta}^{p} \chi_{1} \chi_{3}+64 / 9 R_{q \pi \eta}^{p} \chi_{13}^{2}+16 / 3 R_{p}^{c} \chi_{p} \chi_{13}+8 / 9\left(R_{p}^{d}\right)^{2}\right]$

$+\bar{A}\left(\chi_{p}\right)^{2}\left[1 / 8 \chi_{13}+1 / 18\left(R_{q \pi \eta}^{p}\right)^{2} \chi_{13}+1 / 54 R_{q \pi \eta}^{p} R_{p}^{c} \chi_{p}-1 / 54\left(R_{p}^{c}\right)^{2} \chi_{p}+1 / 72\left(R_{p}^{c}\right)^{2} \chi_{13}\right]$

$+\bar{A}\left(\chi_{p}\right) \bar{A}\left(\chi_{p s}\right)\left[1 / 72 R_{q \pi \eta}^{p} \chi_{p}+19 / 216 R_{q \pi \eta}^{p} \chi_{q}-5 / 216 R_{s \pi \eta}^{p} \chi_{p}-1 / 24 R_{s \pi \eta}^{p} \chi_{q}+1 / 27 R_{p}^{c} \chi_{p}\right]$

$+\bar{A}\left(\chi_{p}\right) \bar{A}\left(\chi_{q s}\right)\left[1 / 18 R_{q \pi \eta}^{p} \chi_{q}-1 / 72 R_{s \pi \eta}^{p} R_{q s p}^{z} \chi_{p}+1 / 216 R_{s \pi \eta}^{p} R_{q s p}^{z} \chi_{q}+1 / 12 R_{p}^{c} \chi_{13}\right]$

$+\bar{A}\left(\chi_{p}\right) \bar{A}\left(\chi_{m}\right)\left[-1 / 27 R_{q m n}^{p} R_{n q q}^{m} \chi_{p}+1 / 9 R_{q m n}^{p} R_{n 13}^{m} \chi_{p}+1 / 27 R_{q m n}^{p} R_{n 13}^{m} \chi_{q}+1 / 27 R_{q m n}^{p} R_{m n 13}^{v} \chi_{p}\right.$

$-1 / 54 R_{q m n}^{p} R_{m n 13}^{v} \chi_{m}+1 / 162\left(R_{s m n}^{p}\right)^{2} R_{n s s}^{m} \chi_{s}-1 / 54 R_{n q q}^{m} R_{p}^{c} \chi_{p}+1 / 216 R_{p}^{c} R_{m n 13}^{v} \chi_{p}$

$\left.+1 / 72 R_{p}^{c} R_{m n 13}^{v} \chi_{q}-1 / 36 R_{p}^{c} R_{m n 13}^{v} \chi_{m}\right]+\bar{A}\left(\chi_{p}\right) \bar{A}\left(\chi_{13}\right)\left[1 / 18 \chi_{p}-1 / 36 \chi_{13}\right]$

$+\bar{A}\left(\chi_{p}\right) \bar{A}\left(\chi_{s t}\right)\left[1 / 162 R_{p p s t}^{u} \chi_{p}-1 / 81 R_{p q s t}^{u} \chi_{p}+1 / 81 R_{p p s t}^{u m} \chi_{p}-1 / 81 R_{p q s t}^{u m} \chi_{p}-1 / 162 R_{p p s t}^{w}\right]$

$+\bar{A}\left(\chi_{p}\right) \bar{B}\left(\chi_{p}, \chi_{p}, 0\right)\left[11 / 54 R_{q \pi \eta}^{p} R_{p}^{c} \chi_{p} \chi_{13}-1 / 27 R_{q \pi \eta}^{p} R_{p}^{c} \chi_{p}^{2}-1 / 18 R_{q \pi \eta}^{p} R_{p}^{c} \chi_{13}^{2}+1 / 54 R_{q \pi \eta}^{p} R_{p}^{d} \chi_{p}\right]$

$+\bar{A}\left(\chi_{p}\right) \bar{B}\left(\chi_{p}, \chi_{m}, 0\right)\left[2 / 27 R_{n 13}^{m} R_{p}^{c} \chi_{p}^{2}+1 / 27 R_{n 13}^{m} R_{p}^{c} \chi_{1} \chi_{3}\right]-\bar{A}\left(\chi_{p}\right) \bar{B}\left(\chi_{q}, \chi_{q}, 0\right)\left[1 / 54 R_{q \pi \eta}^{p} R_{q}^{d} \chi_{q}\right.$

$\left.+1 / 36 R_{p}^{c} R_{q}^{d} \chi_{13}\right]+2 / 27 \bar{A}\left(\chi_{p}\right) \bar{B}\left(\chi_{1}, \chi_{3}, 0\right) R_{p \pi \eta}^{q} R_{p}^{c} \chi_{p} \chi_{13}+1 / 27 \bar{A}\left(\chi_{p}\right) \bar{B}\left(\chi_{1}, \chi_{3}, 0, k\right) R_{p \pi \eta}^{q} R_{p}^{c} \chi_{p}$

$+\bar{A}\left(\chi_{p}, \varepsilon\right) \pi_{16}\left[-1 / 4 \bar{\chi}_{1} R_{q \pi \eta}^{p} \chi_{13}+1 / 8 \bar{\chi}_{1} R_{p}^{c} \chi_{13}+5 / 18 R_{q \pi \eta}^{p} \chi_{p} \chi_{13}+7 / 36 R_{p}^{c} \chi_{p} \chi_{13}+5 / 72 R_{p}^{c} \chi_{q} \chi_{13}\right.$

$\left.-1 / 72 R_{p}^{d} \chi_{13}\right]+\bar{A}\left(\chi_{p s}\right) \pi_{16}\left[-1 / 4 \chi_{p} \chi_{13}+1 / 8 \chi_{13} \chi_{s}+1 / 4 \chi_{13}^{2}+3 / 8 \bar{\chi}_{1} \chi_{13}\right]+4 \bar{A}\left(\chi_{p s}\right) L_{0}^{r} \chi_{p s} \chi_{13}$

$+10 \bar{A}\left(\chi_{p s}\right) L_{3}^{r} \chi_{p s} \chi_{13}-8 \bar{A}\left(\chi_{p s}\right) L_{5}^{r} \chi_{p s} \chi_{13}+16 \bar{A}\left(\chi_{p s}\right) L_{8}^{r} \chi_{p s} \chi_{13}-1 / 4 \bar{A}\left(\chi_{p s}\right) \bar{A}\left(\chi_{q t}\right) \chi_{13}$

$+\bar{A}\left(\chi_{p s}\right) \bar{A}\left(\chi_{m}\right)\left[1 / 27 R_{n p p}^{m} \chi_{p}-5 / 216 R_{n p s}^{m} \chi_{p}-1 / 24 R_{n p s}^{m} \chi_{q}+1 / 12 R_{n q q}^{m} \chi_{13}-5 / 72 R_{n q s}^{m} \chi_{p}\right.$

$\left.-19 / 216 R_{n q s}^{m} R_{p s m}^{z} \chi_{q}+1 / 72 R_{n 13}^{m} \chi_{p}\right]+\bar{A}\left(\chi_{p s}\right) \bar{B}\left(\chi_{p}, \chi_{m}, 0\right)\left[-2 / 9 R_{n q s}^{m} \chi_{p} \chi_{13}-2 / 27 R_{n q s}^{m} \chi_{p} \chi_{s}\right.$

$\left.-1 / 27 R_{n q s}^{m} \chi_{q} \chi_{s}-1 / 27 R_{n 13}^{m} \chi_{p}^{2}+1 / 27 R_{n 13}^{m} \chi_{1} \chi_{3}\right]+1 / 12 \bar{A}\left(\chi_{p s}\right) \bar{B}\left(\chi_{q}, \chi_{q}, 0\right) R_{q}^{d} \chi_{13}$

- $\bar{A}\left(\chi_{p s}\right) \bar{B}\left(\chi_{1}, \chi_{3}, 0\right)\left[1 / 27 R_{p \pi \eta}^{q} \chi_{p}^{2}+4 / 27 R_{s \pi \eta}^{q} \chi_{p s} \chi_{13}+1 / 27 R_{s \pi \eta}^{q} \chi_{1} \chi_{3}\right]$

$+\bar{A}\left(\chi_{p s}\right) \bar{B}\left(\chi_{1}, \chi_{3}, 0, k\right)\left[1 / 27 R_{p \pi \eta}^{q} \chi_{q}-2 / 27 R_{s \pi \eta}^{q} \chi_{p s}-2 / 27 R_{s \pi \eta}^{q} \chi_{13}\right]-\bar{A}\left(\chi_{p s}, \varepsilon\right) \pi_{16}\left[3 / 8 \chi_{13} \chi_{s}\right.$

$\left.+5 / 12 \chi_{13}^{2}+3 / 8 \bar{\chi}_{1} \chi_{13}\right]+\bar{A}\left(\chi_{m}\right) \pi_{16}\left[-1 / 8 \bar{\chi}_{1} R_{m n 13}^{v} \chi_{13}+1 / 24 R_{m n 13}^{v} \chi_{m} \chi_{13}-1 / 12 R_{m n 13}^{v} \chi_{13}^{2}\right]$

$-\bar{A}\left(\chi_{m}\right) L_{0}^{r}\left[8 R_{n 13}^{m} \chi_{m} \chi_{13}+4 / 3 R_{m n 13}^{v} \chi_{m} \chi_{13}\right]+16 \bar{A}\left(\chi_{m}\right) L_{1}^{r} \chi_{m} \chi_{13}+4 \bar{A}\left(\chi_{m}\right) L_{2}^{r} \chi_{m} \chi_{13}$

$-\bar{A}\left(\chi_{m}\right) L_{3}^{r}\left[8 R_{n 13}^{m} \chi_{m} \chi_{13}+10 / 3 R_{m n 13}^{v} \chi_{m} \chi_{13}\right]-16 \bar{A}\left(\chi_{m}\right) L_{4}^{r}\left[\chi_{m} \chi_{13}-\bar{\chi}_{1} R_{n 13}^{m} \chi_{13}\right]$

$+\bar{A}\left(\chi_{m}\right) L_{5}^{r}\left[16 / 3 R_{n 13}^{m} \chi_{m} \chi_{13}+16 / 3 R_{n 13}^{m} \chi_{13}^{2}+8 / 3 R_{m n 13}^{v} \chi_{m} \chi_{13}\right]+16 \bar{A}\left(\chi_{m}\right) L_{6}^{r}\left[\chi_{m} \chi_{13}\right.$

$\left.-\bar{\chi}_{1} R_{n 13}^{m} \chi_{13}\right]+\bar{A}\left(\chi_{m}\right) L_{7}^{r}\left[32 R_{n 13}^{m} \chi_{m} \chi_{13}-32 / 3 R_{n 13}^{m} \chi_{1} \chi_{3}-64 / 3 R_{n 13}^{m} \chi_{13}^{2}-16 / 3 R_{p}^{d} R_{m 456 n p}^{z}\right.$

$\left.+16 / 3 R_{q}^{d} R_{m 456 n p}^{z}-8 / 3\left(R_{m 456 n p}^{z}\right)^{2}+16 / 3 R_{m 456 n 1}^{z} R_{m 456 n 3}^{z}\right]+\bar{A}\left(\chi_{m}\right) L_{8}^{r}\left[-128 / 9 R_{n 13}^{m} \chi_{1} \chi_{3}\right.$

$-64 / 9 R_{n 13}^{m} \chi_{13}^{2}-16 / 9 R_{p}^{d} R_{m 456 n p}^{z}+16 / 9 R_{q}^{d} R_{m 456 n p}^{z}-8 / 9\left(R_{m 456 n p}^{z}\right)^{2}+16 / 9 R_{m 456 n 1}^{z} R_{m 456 n 3}^{z}$

$\left.-8 / 3 R_{m n 13}^{v} \chi_{m}^{2}-8 / 3 R_{m n 13}^{v} \chi_{1} \chi_{3}\right]+\bar{A}\left(\chi_{m}\right)^{2}\left[1 / 81 R_{s m n}^{p} R_{n p s}^{m} R_{n s s}^{m} \chi_{s}-1 / 81 R_{s m n}^{q} R_{n p s}^{m} R_{n s s}^{m} \chi_{s}\right.$

$+1 / 18\left(R_{n 13}^{m}\right)^{2} \chi_{13}-1 / 54 R_{n 13}^{m} R_{m n 13}^{v} \chi_{m}+1 / 54 R_{n 13}^{m} R_{m n 13}^{v} \chi_{13}+1 / 162\left(R_{n s s}^{m}\right)^{2} R_{m n 13}^{v} \chi_{s}$

$\left.-1 / 54\left(R_{m n 13}^{v}\right)^{2} \chi_{m}+1 / 72\left(R_{m n 13}^{v}\right)^{2} \chi_{13}\right]-\bar{A}\left(\chi_{m}\right) \bar{A}\left(\chi_{s t}\right)\left[1 / 27 R_{n 13}^{m} R_{m n s t}^{v} \chi_{13}+1 / 81 R_{p q s t}^{u m} \chi_{q}\right.$

$\left.+1 / 162 R_{m n 13}^{v} R_{m n s t}^{v} \chi_{m}+1 / 81 R_{p p s t}^{w m}-1 / 81 R_{p q s t}^{w m}+1 / 162 R_{p p s t}^{w m m}-1 / 81 R_{13 s t}^{w m m}\right]$

$+\bar{A}\left(\chi_{m}\right) \bar{B}\left(\chi_{p}, \chi_{p}, 0\right)\left[1 / 9 R_{q m n}^{p} R_{n p p}^{m} \chi_{p}^{2}-1 / 54 R_{n p p}^{m} R_{p}^{d} \chi_{p}-1 / 27 R_{n 13}^{m} R_{p}^{d} \chi_{p}-1 / 72 R_{p}^{d} R_{m n 13}^{v} \chi_{p}\right.$

$\left.+1 / 72 R_{p}^{d} R_{m n 13}^{v} \chi_{q}-1 / 36 R_{p}^{d} R_{m n 13}^{v} \chi_{m}\right]+\bar{A}\left(\chi_{m}\right) \bar{B}\left(\chi_{p}, \chi_{m}, 0\right)\left[-2 / 81 R_{s m n}^{p} R_{n q s}^{m} R_{n s s}^{m} \chi_{p} \chi_{s}\right.$

$\left.-1 / 81 R_{s m n}^{p} R_{n q s}^{m} R_{n s s}^{m} \chi_{q} \chi_{s}+2 / 27 R_{n p p}^{m} R_{n 13}^{m} \chi_{p}^{2}+1 / 27 R_{n p p}^{m} R_{n 13}^{m} \chi_{1} \chi_{3}\right]$

$+\bar{A}\left(\chi_{m}\right) \bar{B}\left(\chi_{p}, \chi_{n}, 0\right)\left[-2 / 81 R_{s m n}^{p} R_{n s s}^{m} R_{m q s}^{n} \chi_{p} \chi_{s}-1 / 81 R_{s m n}^{p} R_{n s s}^{m} R_{m q s}^{n} \chi_{q} \chi_{s}\right.$

$\left.+2 / 27 R_{n p p}^{m} R_{m 13}^{n} \chi_{p}^{2}+1 / 27 R_{n p p}^{m} R_{m 13}^{n} \chi_{1} \chi_{3}\right]-1 / 27 \bar{A}\left(\chi_{m}\right) \bar{B}\left(\chi_{m}, \chi_{m}, 0\right) R_{n 1 s}^{m} R_{n 3 s}^{m} R_{n s s}^{m} \chi_{13} \chi_{s}$

- $\bar{A}\left(\chi_{m}\right) \bar{B}\left(\chi_{m}, \chi_{n}, 0\right)\left[1 / 81 R_{n p s}^{m} R_{n s s}^{m} R_{m p s}^{n} \chi_{p} \chi_{s}+2 / 81 R_{n p s}^{m} R_{n s s}^{m} R_{m q s}^{n} \chi_{13} \chi_{s}\right]$

$+1 / 81 \bar{A}\left(\chi_{m}\right) \bar{B}\left(\chi_{m}, \chi_{n}, 0, k\right)\left[R_{n p s}^{m} R_{n s s}^{m} R_{m p s}^{n} \chi_{s}-R_{n p s}^{m} R_{n s s}^{m} R_{m q s}^{n} \chi_{s}\right]$

- $1 / 27 \bar{A}\left(\chi_{m}\right) \bar{B}\left(\chi_{n}, \chi_{n}, 0\right) R_{n s s}^{m} R_{m 1 s}^{n} R_{m 3 s}^{n} \chi_{13} \chi_{s}+\bar{A}\left(\chi_{m}\right) \bar{B}\left(\chi_{1}, \chi_{3}, 0\right)\left[2 / 27 R_{p m n}^{q} R_{n p p}^{m} \chi_{p} \chi_{13}\right.$ 
$\left.-2 / 81 R_{n s s}^{m} R_{s m n}^{1} R_{s m n}^{3} \chi_{13} \chi_{s}\right]+\bar{A}\left(\chi_{m}\right) \bar{B}\left(\chi_{1}, \chi_{3}, 0, k\right)\left[1 / 27 R_{p m n}^{q} R_{n p p}^{m} \chi_{p}-1 / 81 R_{n s s}^{m} R_{s m n}^{1} R_{s m n}^{3} \chi_{s}\right]$

$+\bar{A}\left(\chi_{m}, \varepsilon\right) \pi_{16}\left[-1 / 4 \chi_{m} \chi_{13}+1 / 8 \bar{\chi}_{1} R_{m n 13}^{v} \chi_{13}+1 / 4 R_{n 13}^{m} \chi_{m} \chi_{13}+5 / 9 R_{n 13}^{m} \chi_{13}^{2}+1 / 8 R_{m n 13}^{v} \chi_{m} \chi_{13}\right.$

$\left.+5 / 36 R_{m n 13}^{v} \chi_{13}^{2}\right]+\bar{A}\left(\chi_{\pi}\right) \bar{A}\left(\chi_{\eta}\right)\left[1 / 270 \bar{\chi}_{1} R_{\pi \eta 13}^{v} R_{\eta \pi 13}^{v}+1 / 45 R_{n 13}^{m} R_{n m 13}^{v} \chi_{n}+1 / 270 R_{n 13}^{m} R_{n m 13}^{v} \chi_{13}\right.$

$-1 / 81 R_{n s s}^{m} R_{\eta p s}^{\pi} R_{\pi p s}^{\eta} \chi_{s}+1 / 81 R_{n s s}^{m} R_{\eta p s}^{\pi} R_{\pi q s}^{\eta} \chi_{s}-29 / 1080 R_{\eta p p}^{\pi} R_{\pi p p}^{\eta} \chi_{p}+1 / 72 R_{\eta p p}^{\pi} R_{\pi p p}^{\eta} \chi_{q}$

$\left.-17 / 540 R_{\eta p p}^{\pi} R_{\pi q q}^{\eta} \chi_{13}+1 / 5 R_{\eta 13}^{\pi} R_{\pi 13}^{\eta} \chi_{13}-1 / 162 R_{\eta s s}^{\pi} R_{\pi s s}^{\eta} R_{m n 13}^{v} \chi_{s}\right]-\bar{A}\left(\chi_{1}\right) \bar{A}\left(\chi_{3}\right)\left[1 / 54 R_{q \pi \eta}^{p} R_{q}^{c} \chi_{q}\right.$

$\left.-1 / 9 R_{3 \pi \eta}^{1} R_{1 \pi \eta}^{3} \chi_{13}+1 / 36 R_{1}^{c} R_{3}^{c} \chi_{13}\right]+8 \bar{A}\left(\chi_{13}\right) L_{1}^{r} \chi_{13}^{2}+20 \bar{A}\left(\chi_{13}\right) L_{2}^{r} \chi_{13}^{2}+32 \bar{A}\left(\chi_{13}\right) L_{6}^{r} \chi_{13}^{2}$

$-1 / 4 \bar{A}\left(\chi_{13}\right)^{2} \chi_{13}+\bar{A}\left(\chi_{13}\right) \bar{B}\left(\chi_{1}, \chi_{3}, 0\right)\left[1 / 9 \chi_{1} \chi_{3}+4 / 9 \chi_{13}^{2}\right]+4 / 9 \bar{A}\left(\chi_{13}\right) \bar{B}\left(\chi_{1}, \chi_{3}, 0, k\right) \chi_{13}$

$-31 / 18 \bar{A}\left(\chi_{13}, \varepsilon\right) \pi_{16} \chi_{13}^{2}-1 / 4 \bar{A}\left(\chi_{1 s}\right) \bar{A}\left(\chi_{3 s}\right) \chi_{13}+32 \bar{A}\left(\chi_{s t}\right) L_{1}^{r} \chi_{13} \chi_{s t}+8 \bar{A}\left(\chi_{s t}\right) L_{2}^{r} \chi_{13} \chi_{s t}$

- $32 \bar{A}\left(\chi_{s t}\right) L_{4}^{r} \chi_{13} \chi_{s t}+32 \bar{A}\left(\chi_{s t}\right) L_{6}^{r} \chi_{13} \chi_{s t}+\bar{A}\left(\chi_{s t}\right) \bar{B}\left(\chi_{p}, \chi_{m}, 0\right)\left[1 / 81 R_{p p s t}^{u m} \chi_{p} \chi_{m}-1 / 81 R_{p q s t}^{u m} \chi_{p} \chi_{m}\right.$

$\left.-1 / 81 R_{p q s t}^{u m} \chi_{1} \chi_{3}+2 / 81 R_{p q s t}^{w m} \chi_{p}+1 / 81 R_{p q s t}^{w m} \chi_{q}\right]-1 / 81 \bar{A}\left(\chi_{s t}\right) \bar{B}\left(\chi_{p}, \chi_{m}, 0, k\right)\left[R_{p p s t}^{u m} \chi_{p}\right.$

$\left.+R_{p q s t}^{u m} \chi_{p}\right]+2 / 9 \bar{A}\left(\chi_{s t}\right) \bar{B}\left(\chi_{m}, \chi_{m}, 0\right) R_{n 13}^{m} R_{n s t}^{m} \chi_{13} \chi_{s t}+\bar{A}\left(\chi_{s t}\right) \bar{B}\left(\chi_{\pi}, \chi_{\eta}, 0\right)\left[1 / 81 R_{p p s t}^{u \pi \eta} \chi_{\pi} \chi_{\eta}\right.$

$\left.-1 / 81 R_{p q s t}^{u \pi \eta} \chi_{\pi} \chi_{\eta}+1 / 81 R_{p p s t}^{w \pi \eta} \chi_{p}+2 / 81 R_{p q s t}^{w \pi \eta} \chi_{13}\right]+\bar{A}\left(\chi_{s t}\right) \bar{B}\left(\chi_{\pi}, \chi_{\eta}, 0, k\right)\left[-1 / 81 R_{p p s t}^{u \pi \eta} \chi_{p}\right.$

$\left.-2 / 81 R_{p q s t}^{u \pi \eta} \chi_{13}-1 / 81 R_{p p s t}^{w \pi \eta}+1 / 81 R_{p q s t}^{w \pi \eta}\right]-\bar{A}\left(\chi_{s t}\right) \bar{B}\left(\chi_{1}, \chi_{3}, 0\right)\left[1 / 81 R_{13 s t}^{u} \chi_{1} \chi_{3}-2 / 81 R_{13 s t}^{w} \chi_{13}\right]$

- $\bar{A}\left(\chi_{s t}\right) \bar{B}\left(\chi_{1}, \chi_{3}, 0, k\right)\left[2 / 81 R_{13 s t}^{u} \chi_{13}-1 / 81 R_{13 s t}^{w}\right]-1 / 2 \bar{A}\left(\chi_{s t}, \varepsilon\right) \pi_{16} \chi_{13} \chi_{s t}$

$-\bar{B}\left(\chi_{p}, \chi_{p}, 0\right) \pi_{16}\left[1 / 8 \bar{\chi}_{1} R_{p}^{d} \chi_{13}+1 / 24 R_{p}^{d} \chi_{q} \chi_{13}\right]-4 / 3 \bar{B}\left(\chi_{p}, \chi_{p}, 0\right) L_{0}^{r} R_{p}^{d} \chi_{p} \chi_{13}$

$-10 / 3 \bar{B}\left(\chi_{p}, \chi_{p}, 0\right) L_{3}^{r} R_{p}^{d} \chi_{p} \chi_{13}+8 \bar{B}\left(\chi_{p}, \chi_{p}, 0\right) L_{4}^{r} \bar{\chi}_{1} R_{q \pi \eta}^{p} \chi_{p} \chi_{13}-\bar{B}\left(\chi_{p}, \chi_{p}, 0\right) L_{5}^{r}\left[16 / 3 R_{q \pi \eta}^{p} \chi_{p} \chi_{13}^{2}\right.$

$\left.-8 R_{q \pi \eta}^{p} \chi_{p}^{2} \chi_{13}\right]-16 \bar{B}\left(\chi_{p}, \chi_{p}, 0\right) L_{6}^{r} \bar{\chi}_{1} R_{q \pi \eta}^{p} \chi_{p} \chi_{13}+\bar{B}\left(\chi_{p}, \chi_{p}, 0\right) L_{8}^{r}\left[32 / 3 R_{q \pi \eta}^{p} \chi_{p} \chi_{13}^{2}-16 R_{q \pi \eta}^{p} \chi_{p}^{2} \chi_{13}\right]$

$+\bar{B}\left(\chi_{p}, \chi_{p}, 0\right)^{2}\left[1 / 8 R_{q \pi \eta}^{p} R_{p}^{d} \chi_{p} \chi_{13}-1 / 72 R_{q \pi \eta}^{p} R_{p}^{d} \chi_{q} \chi_{13}\right]+\bar{B}\left(\chi_{p}, \chi_{p}, 0\right) \bar{B}\left(\chi_{p}, \chi_{m}, 0\right)\left[2 / 27 R_{n 13}^{m} R_{p}^{d} \chi_{p}^{2}\right.$

$\left.+1 / 27 R_{n 13}^{m} R_{p}^{d} \chi_{1} \chi_{3}\right]+2 / 27 \bar{B}\left(\chi_{p}, \chi_{p}, 0\right) \bar{B}\left(\chi_{1}, \chi_{3}, 0\right) R_{p \pi \eta}^{q} R_{p}^{d} \chi_{p} \chi_{13}$

$+1 / 27 \bar{B}\left(\chi_{p}, \chi_{p}, 0\right) \bar{B}\left(\chi_{1}, \chi_{3}, 0, k\right) R_{p \pi \eta}^{q} R_{p}^{d} \chi_{p}+\bar{B}\left(\chi_{p}, \chi_{p}, 0, \varepsilon\right) \pi_{16}\left[1 / 8 \bar{\chi}_{1} R_{p}^{d} \chi_{13}+1 / 8 R_{p}^{d} \chi_{p} \chi_{13}\right.$

$\left.+5 / 36 R_{p}^{d} \chi_{13}^{2}\right]+\bar{B}\left(\chi_{p}, \chi_{m}, 0\right) L_{7}^{r}\left[32 / 3 R_{p}^{d} R_{m 456 n q}^{z} \chi_{p}+16 / 3 R_{p}^{d} R_{m 456 n q}^{z} \chi_{q}\right]$

$+\bar{B}\left(\chi_{p}, \chi_{m}, 0\right) L_{8}^{r}\left[32 / 9 R_{p}^{d} R_{m 456 n q}^{z} \chi_{p}+16 / 9 R_{p}^{d} R_{m 456 n q}^{z} \chi_{q}\right]+8 \bar{B}\left(\chi_{m}, \chi_{m}, 0\right) L_{4}^{r} \bar{\chi}_{1} R_{n 13}^{m} \chi_{m} \chi_{13}$

$+8 / 3 \bar{B}\left(\chi_{m}, \chi_{m}, 0\right) L_{5}^{r} R_{n 13}^{m} \chi_{m}^{2} \chi_{13}-16 \bar{B}\left(\chi_{m}, \chi_{m}, 0\right) L_{6}^{r} \bar{\chi}_{1} R_{n 13}^{m} \chi_{m} \chi_{13}$

$+16 \bar{B}\left(\chi_{m}, \chi_{m}, 0\right) L_{7}^{r} R_{m 456 n 1}^{z} R_{m 456 n 3}^{z} \chi_{13}-\bar{B}\left(\chi_{m}, \chi_{m}, 0\right) L_{8}^{r}\left[16 / 3 R_{n 13}^{m} \chi_{m}^{2} \chi_{13}\right.$

$\left.-16 / 3 R_{m 456 n 1}^{z} R_{m 456 n 3}^{z} \chi_{13}\right]+\bar{B}\left(\chi_{\pi}, \chi_{\eta}, 0\right) L_{7}^{r}\left[16 / 3 R_{\pi 456 \eta p}^{z} R_{\eta 456 \pi p}^{z} \chi_{p}+32 / 3 R_{\pi 456 \eta p}^{z} R_{\eta 456 \pi q}^{z} \chi_{13}\right]$

$+\bar{B}\left(\chi_{\pi}, \chi_{\eta}, 0\right) L_{8}^{r}\left[16 / 9 R_{\pi 456 \eta p}^{z} R_{\eta 456 \pi p}^{z} \chi_{p}+32 / 9 R_{\pi 456 \eta p}^{z} R_{\eta 456 \pi q}^{z} \chi_{13}\right]$

$-16 / 3 \bar{B}\left(\chi_{\pi}, \chi_{\eta}, 0, k\right) L_{7}^{r}\left[R_{\pi 456 \eta p}^{z} R_{\eta 456 \pi p}^{z}-R_{\pi 456 \eta p}^{z} R_{\eta 456 \pi q}^{z}\right]-16 / 9 \bar{B}\left(\chi_{\pi}, \chi_{\eta}, 0, k\right) L_{8}^{r}\left[R_{\pi 456 \eta p}^{z} R_{\eta 456 \pi p}^{z}\right.$

- $\left.R_{\pi 456 \eta p}^{z} R_{\eta 456 \pi q}^{z}\right]-1 / 36 \bar{B}\left(\chi_{1}, \chi_{1}, 0\right) \bar{B}\left(\chi_{3}, \chi_{3}, 0\right) R_{1}^{d} R_{3}^{d} \chi_{13}+32 / 3 \bar{B}\left(\chi_{1}, \chi_{3}, 0\right) L_{7}^{r} R_{1}^{d} R_{3}^{d} \chi_{13}$

$+32 / 9 \bar{B}\left(\chi_{1}, \chi_{3}, 0\right) L_{8}^{r} R_{1}^{d} R_{3}^{d} \chi_{13}+16 / 3 \bar{B}\left(\chi_{1}, \chi_{3}, 0, k\right) L_{7}^{r} R_{1}^{d} R_{3}^{d}+16 / 9 \bar{B}\left(\chi_{1}, \chi_{3}, 0, k\right) L_{8}^{r} R_{1}^{d} R_{3}^{d}$

$+H^{F}\left(1, \chi_{p}, \chi_{p}, \chi_{13}, \chi_{13}\right)\left[-1 / 4 \chi_{p} \chi_{13}-3 / 8 \chi_{13}^{2}-1 / 18\left(R_{q \pi \eta}^{p}\right)^{2} \chi_{13}^{2}+1 / 6 R_{q \pi \eta}^{p} R_{p}^{c} \chi_{13}^{2}-1 / 24\left(R_{p}^{c}\right)^{2} \chi_{13}^{2}\right]$

$+H^{F}\left(1, \chi_{p}, \chi_{1 s}, \chi_{3 s}, \chi_{13}\right)\left[-5 / 12 R_{q \pi \eta}^{p} \chi_{q} \chi_{13}+1 / 6 R_{q \pi \eta}^{p} \chi_{p s} \chi_{13}+1 / 8 R_{s \pi \eta}^{p} \chi_{q} \chi_{13}+7 / 24 R_{s \pi \eta}^{p} \chi_{13} \chi_{s}\right.$

$\left.-1 / 12 R_{p}^{c} \chi_{p s} \chi_{13}\right]+1 / 4 H^{F}\left(1, \chi_{p s}, \chi_{q t}, \chi_{s t}, \chi_{13}\right) \chi_{13} \chi_{s t}+H^{F}\left(1, \chi_{m}, \chi_{p}, \chi_{13}, \chi_{13}\right)\left[2 / 9 R_{q m n}^{p} R_{n 13}^{m} \chi_{13}^{2}\right.$

$\left.-1 / 18 R_{q m n}^{p} R_{m n 13}^{v} \chi_{13}^{2}+1 / 9 R_{n p p}^{m} R_{p}^{c} \chi_{13}^{2}+1 / 36 R_{p}^{c} R_{m n 13}^{v} \chi_{13}^{2}\right]$

$+H^{F}\left(1, \chi_{m}, \chi_{m}, \chi_{13}, \chi_{13}\right)\left[2 / 9\left(R_{n 13}^{m}\right)^{2} \chi_{13}^{2}+2 / 9 R_{n 13}^{m} R_{m n 13}^{v} \chi_{13}^{2}+5 / 72\left(R_{m n 13}^{v}\right)^{2} \chi_{13}^{2}\right]$

$+H^{F}\left(1, \chi_{m}, \chi_{1 s}, \chi_{3 s}, \chi_{13}\right)\left[-1 / 8 R_{n p s}^{m} \chi_{p} \chi_{13}-5 / 24 R_{n p s}^{m} \chi_{q} \chi_{13}+1 / 3 R_{n 13}^{m} \chi_{13}^{2}-1 / 24 R_{m n p s}^{v} \chi_{m} \chi_{13}\right.$

$\left.-1 / 24 R_{m n 13}^{v} \chi_{13} \chi_{s}\right]+H^{F}\left(1, \chi_{\pi}, \chi_{\eta}, \chi_{13}, \chi_{13}\right)\left[1 / 9 R_{\eta p p}^{\pi} R_{\pi p p}^{\eta} \chi_{13}^{2}+2 / 9 R_{\eta 13}^{\pi} R_{\pi 13}^{\eta} \chi_{13}^{2}\right.$

$\left.+1 / 36 R_{\pi \eta 13}^{v} R_{\eta \pi 13}^{v} \chi_{13}^{2}\right]+H^{F}\left(1, \chi_{1}, \chi_{13}, \chi_{3}, \chi_{13}\right)\left[-1 / 18 R_{q \pi \eta}^{p} R_{q}^{c} \chi_{13}^{2}+1 / 3 R_{3 \pi \eta}^{1} R_{1 \pi \eta}^{3} \chi_{13}^{2}\right.$

$\left.+1 / 36 R_{1}^{c} R_{3}^{c} \chi_{13}^{2}\right]+1 / 4 H^{F}\left(1, \chi_{13}, \chi_{13}, \chi_{13}, \chi_{13}\right) \chi_{13}^{2}-H^{F}\left(2, \chi_{p}, \chi_{p}, \chi_{13}, \chi_{13}\right)\left[1 / 18 R_{q \pi \eta}^{p} R_{p}^{d} \chi_{13}^{2}\right.$

$\left.-5 / 36 R_{p}^{c} R_{p}^{d} \chi_{13}^{2}\right]+H^{F}\left(2, \chi_{p}, \chi_{m}, \chi_{13}, \chi_{13}\right)\left[1 / 9 R_{n p p}^{m} R_{p}^{d} \chi_{13}^{2}+1 / 36 R_{p}^{d} R_{m n 13}^{v} \chi_{13}^{2}\right]$

$-H^{F}\left(2, \chi_{p}, \chi_{13}, \chi_{q}, \chi_{13}\right)\left[1 / 18 R_{p \pi \eta}^{q} R_{p}^{d} \chi_{13}^{2}-1 / 36 R_{q}^{c} R_{p}^{d} \chi_{13}^{2}\right]-1 / 12 H^{F}\left(2, \chi_{p}, \chi_{1 s}, \chi_{3 s}, \chi_{13}\right) R_{p}^{d} \chi_{p s} \chi_{13}$

$+5 / 72 H^{F}\left(5, \chi_{p}, \chi_{p}, \chi_{13}, \chi_{13}\right)\left(R_{p}^{d}\right)^{2} \chi_{13}^{2}+1 / 36 H^{F}\left(5, \chi_{1}, \chi_{3}, \chi_{13}, \chi_{13}\right) R_{1}^{d} R_{3}^{d} \chi_{13}^{2}$ 


$$
\begin{aligned}
& +H_{1}^{F}\left(1, \chi_{p}, \chi_{p}, \chi_{13}, \chi_{13}\right)\left[2 \chi_{13}^{2}+4 / 9\left(R_{q \pi \eta}^{p}\right)^{2} \chi_{13}^{2}-4 / 9 R_{q \pi \eta}^{p} R_{p}^{c} \chi_{13}^{2}+2 / 9\left(R_{p}^{c}\right)^{2} \chi_{13}^{2}\right] \\
& +2 / 3 H_{1}^{F}\left(1, \chi_{p}, \chi_{1 s}, \chi_{3 s}, \chi_{13}\right) R_{q \pi \eta}^{p} R_{s q p}^{z} \chi_{13}^{2}-2 / 3 H_{1}^{F}\left(1, \chi_{p s}, \chi_{q s}, \chi_{p}, \chi_{13}\right) R_{s \pi \eta}^{p} \chi_{13}^{2} \\
& -2 / 3 H_{1}^{F}\left(1, \chi_{p s}, \chi_{q s}, \chi_{m}, \chi_{13}\right) R_{n 13}^{m} R_{s p m}^{z} \chi_{13}^{2}+2 / 9 H_{1}^{F}\left(1, \chi_{13}, \chi_{p}, \chi_{m}, \chi_{13}\right)\left[R_{q m n}^{p} R_{m n 13}^{v} \chi_{13}^{2}\right. \\
& \left.-R_{n p p}^{m} R_{q p m}^{z} R_{p}^{c} \chi_{13}^{2}\right]-H_{1}^{F}\left(1, \chi_{13}, \chi_{m}, \chi_{m}, \chi_{13}\right)\left[2 / 9 R_{n 13}^{m} R_{m n 13}^{v} \chi_{13}^{2}+1 / 9\left(R_{m n 13}^{v}\right)^{2} \chi_{13}^{2}\right] \\
& \text { - } 2 / 9 H_{1}^{F}\left(1, \chi_{13}, \chi_{\pi}, \chi_{\eta}, \chi_{13}\right)\left[R_{\eta p p}^{\pi} R_{q \eta p}^{z} R_{\eta \pi 13}^{v} \chi_{13}^{2}-R_{\eta 13}^{\pi} R_{\eta \pi 13}^{v} \chi_{13}^{2}\right] \\
& +H_{1}^{F}\left(1, \chi_{13}, \chi_{1}, \chi_{3}, \chi_{13}\right)\left[2 / 9 R_{q \pi \eta}^{p} R_{q}^{c} \chi_{13}^{2}-4 / 9 R_{3 \pi \eta}^{1} R_{1 \pi \eta}^{3} \chi_{13}^{2}\right]+2 / 9 H_{1}^{F}\left(3, \chi_{13}, \chi_{p}, \chi_{p}, \chi_{13}\right)\left[R_{q \pi \eta}^{p} R_{p}^{d} \chi_{13}^{2}\right. \\
& \left.-R_{p}^{c} R_{p}^{d} \chi_{13}^{2}\right]+2 / 9 H_{1}^{F}\left(3, \chi_{13}, \chi_{p}, \chi_{q}, \chi_{13}\right) R_{p \pi \eta}^{q} R_{p}^{d} \chi_{13}^{2}+2 / 9 H_{1}^{F}\left(3, \chi_{13}, \chi_{p}, \chi_{m}, \chi_{13}\right) R_{n 13}^{m} R_{p q m}^{z} R_{p}^{d} \chi_{13}^{2} \\
& -1 / 9 H_{1}^{F}\left(7, \chi_{13}, \chi_{p}, \chi_{p}, \chi_{13}\right)\left(R_{p}^{d}\right)^{2} \chi_{13}^{2}-3 / 4 H_{21}^{F}\left(1, \chi_{p}, \chi_{p}, \chi_{13}, \chi_{13}\right) \chi_{13}^{2} \\
& -1 / 4 H_{21}^{F}\left(1, \chi_{p}, \chi_{1 s}, \chi_{3 s}, \chi_{13}\right) R_{q \pi \eta}^{p} R_{s q p}^{z} \chi_{13}^{2}+1 / 4 H_{21}^{F}\left(1, \chi_{p s}, \chi_{q s}, \chi_{p}, \chi_{13}\right)\left[R_{q \pi \eta}^{p} \chi_{13}^{2}+R_{s \pi \eta}^{p} \chi_{13}^{2}-R_{p}^{c} \chi_{13}^{2}\right] \\
& +1 / 4 H_{21}^{F}\left(1, \chi_{p s}, \chi_{q s}, \chi_{q}, \chi_{13}\right) R_{p \pi \eta}^{q} R_{s p q}^{z} \chi_{13}^{2}+1 / 4 H_{21}^{F}\left(1, \chi_{p s}, \chi_{q s}, \chi_{m}, \chi_{13}\right) R_{n 13}^{m} R_{p q m}^{z} R_{s p m}^{z} \chi_{13}^{2} \\
& -1 / 4 H_{21}^{F}\left(1, \chi_{m}, \chi_{1 s}, \chi_{3 s}, \chi_{13}\right) R_{n 13}^{m} R_{s 1 m}^{z} R_{s 3 m}^{z} \chi_{13}^{2}+H_{21}^{F}\left(1, \chi_{13}, \chi_{p}, \chi_{p}, \chi_{13}\right)\left[3 / 8 \chi_{13}^{2}+1 / 6\left(R_{q \pi \eta}^{p}\right)^{2} \chi_{13}^{2}\right. \\
& \left.-1 / 6 R_{q \pi \eta}^{p} R_{p}^{c} \chi_{13}^{2}+1 / 24\left(R_{p}^{c}\right)^{2} \chi_{13}^{2}\right]-H_{21}^{F}\left(1, \chi_{13}, \chi_{p}, \chi_{m}, \chi_{13}\right)\left[1 / 6 R_{q m n}^{p} R_{m n 13}^{v} \chi_{13}^{2}-1 / 12 R_{p}^{c} R_{m n 13}^{v} \chi_{13}^{2}\right] \\
& +1 / 24 H_{21}^{F}\left(1, \chi_{13}, \chi_{m}, \chi_{m}, \chi_{13}\right)\left(R_{m n 13}^{v}\right)^{2} \chi_{13}^{2}+1 / 12 H_{21}^{F}\left(1, \chi_{13}, \chi_{\pi}, \chi_{\eta}, \chi_{13}\right) R_{\pi \eta 13}^{v} R_{\eta \pi 13}^{v} \chi_{13}^{2} \\
& +H_{21}^{F}\left(1, \chi_{13}, \chi_{1}, \chi_{3}, \chi_{13}\right)\left[-1 / 6 R_{q \pi \eta}^{p} R_{q}^{c} \chi_{13}^{2}+1 / 3 R_{3 \pi \eta}^{1} R_{1 \pi \eta}^{3} \chi_{13}^{2}+1 / 12 R_{1}^{c} R_{3}^{c} \chi_{13}^{2}\right] \\
& +3 / 4 H_{21}^{F}\left(1, \chi_{13}, \chi_{13}, \chi_{13}, \chi_{13}\right) \chi_{13}^{2}+3 / 4 H_{21}^{F}\left(1, \chi_{s t}, \chi_{p s}, \chi_{q t}, \chi_{13}\right) \chi_{13}^{2} \\
& -1 / 4 H_{21}^{F}\left(3, \chi_{p s}, \chi_{p}, \chi_{q s}, \chi_{13}\right) R_{p}^{d} \chi_{13}^{2}-H_{21}^{F}\left(3, \chi_{13}, \chi_{p}, \chi_{p}, \chi_{13}\right)\left[1 / 6 R_{q \pi \eta}^{p} R_{p}^{d} \chi_{13}^{2}-1 / 12 R_{p}^{c} R_{p}^{d} \chi_{13}^{2}\right] \\
& -H_{21}^{F}\left(3, \chi_{13}, \chi_{p}, \chi_{q}, \chi_{13}\right)\left[1 / 6 R_{p \pi \eta}^{q} R_{p}^{d} \chi_{13}^{2}-1 / 12 R_{q}^{c} R_{p}^{d} \chi_{13}^{2}\right]+1 / 12 H_{21}^{F}\left(3, \chi_{13}, \chi_{p}, \chi_{m}, \chi_{13}\right) R_{p}^{d} R_{m n 13}^{v} \chi_{13}^{2} \\
& +1 / 24 H_{21}^{F}\left(7, \chi_{13}, \chi_{p}, \chi_{p}, \chi_{13}\right)\left(R_{p}^{d}\right)^{2} \chi_{13}^{2}+1 / 12 H_{21}^{F}\left(7, \chi_{13}, \chi_{1}, \chi_{3}, \chi_{13}\right) R_{1}^{d} R_{3}^{d} \chi_{13}^{2} \text {. }
\end{aligned}
$$

\section{ANALYTICAL RESULTS FOR THE DECAY CONSTANTS}

In the previous section, explicit analytical results for the masses of charged pseudoscalar mesons to NNLO in $\mathrm{PQ} \chi \mathrm{PT}$ were presented for all possible degrees of degeneracy in the quark masses. The decay constants of the pseudoscalar mesons are similarly known, and have for the most part been published earlier in Ref. [10]. In that reference, analytical results were presented for all cases except for the most difficult one with $d_{\mathrm{val}}=2$ and $d_{\text {sea }}=3$. However, the more advanced simplification methods used for the meson masses in this paper has also made it possible to bring that expression down to a manageable size. This is not surprising, since the results for the decay constants are in general slightly less complex than the ones for the meson masses. In order to obtain a complete set of NNLO calculations, the expression for the decay constant with $d_{\mathrm{val}}=2$ and $d_{\text {sea }}=3$ is presented here.

The decay constants $F_{a}$ of the pseudoscalar mesons are obtained from the definition

$$
\left\langle 0\left|A_{a}^{\mu}(0)\right| \phi_{a}(p)\right\rangle=i \sqrt{2} p^{\mu} F_{a},
$$

in terms of the axial current operator $A_{a}^{\mu}(0)$. In the following developments, the flavor index $a$ has been suppressed for simplicity. The Feynman diagrams that contribute to the axial current operator at NNLO, or $\mathcal{O}\left(p^{6}\right)$, are those shown in Fig. 2] Diagrams of $\mathcal{O}\left(p^{2}\right)$ and $\mathcal{O}\left(p^{4}\right)$ also contribute to Eq. (63) via the renormalization of the pseudoscalar meson wave function $\phi_{a}(p)$. From Eq. (63), the expression for the decay constant of a pseudoscalar meson to $\mathcal{O}\left(p^{6}\right)$ is given by

$$
\begin{aligned}
\frac{F_{\text {phys }}}{\sqrt{Z}} & =F_{0}+F_{4}\left(M_{\text {phys }}^{2}, \chi_{i}\right) \\
& +F_{6}\left(M_{\text {phys }}^{2}, \chi_{i}\right)+\mathcal{O}\left(p^{8}\right),
\end{aligned}
$$

where $\sqrt{Z}$ is the wave function renormalization factor. Here the subscripts of the matrix elements $F$ indicate the chiral order, and should not be confused with the flavor index $a$ in Eq. (63). Thus $F_{4}$ and $F_{6}$ denote the matrix elements of the axial current operator at NLO and NNLO, respectively. In the above equation, the lowest order contribution $F_{2}$ has already been identified with $F_{0}$. The wave function renormalization is given in terms of the self-energy diagrams by

$$
Z^{-1} \equiv 1-\left.\frac{\partial \Sigma\left(p^{2}, \chi_{i}\right)}{\partial p^{2}}\right|_{M_{\mathrm{phys}}^{2}}
$$

which becomes, when expanded such that all contributions up to $\mathcal{O}\left(p^{6}\right)$ are taken into account,

$$
\begin{aligned}
\sqrt{Z} & =1+\frac{\Sigma^{\prime}}{2}+\frac{3}{8} \Sigma^{\prime 2}+\cdots \\
\Sigma^{\prime} & =\left.\frac{\partial \Sigma_{4}\left(p^{2}, \chi_{i}\right)}{\partial p^{2}}\right|_{M_{\text {phys }}^{2}}+\left.\frac{\partial \Sigma_{6}\left(p^{2}, \chi_{i}\right)}{\partial p^{2}}\right|_{M_{\text {phys }}^{2}}
\end{aligned}
$$




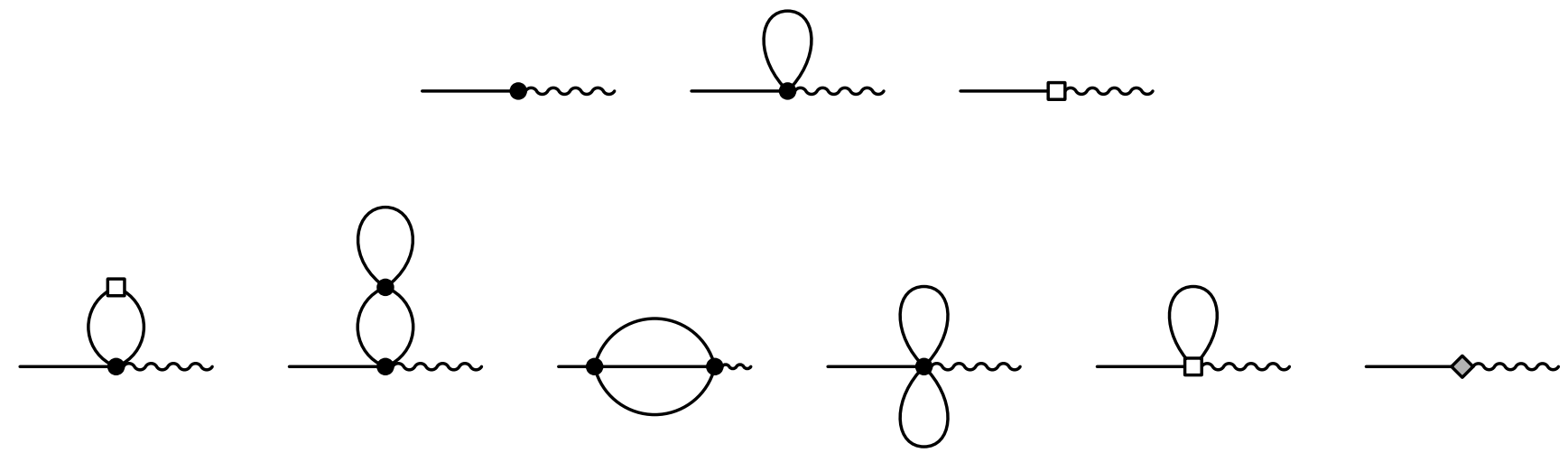

FIG. 2: Feynman diagrams up to $\mathcal{O}\left(p^{6}\right)$ or two loops, for the matrix element $F\left(M_{\text {phys }}^{2}, \chi_{i}\right)$ of the axial current operator $A_{a}^{\mu}(0)$. Filled circles denote vertices of the $\mathcal{L}_{2}$ Lagrangian, whereas open squares and diamonds denote vertices of the $\mathcal{L}_{4}$ and $\mathcal{L}_{6}$ Lagrangians, respectively. In the top row, the first diagram from the left is of $\mathcal{O}\left(p^{2}\right)$, whereas the other two diagrams are of $\mathcal{O}\left(p^{4}\right)$. The diagrams of $\mathcal{O}\left(p^{6}\right)$, which give the NNLO correction to the decay constant, are shown in the bottom row.

and is thus seen to contain self-energy contributions of $\mathcal{O}\left(p^{4}\right)$ as well as $\mathcal{O}\left(p^{6}\right)$. It should be noted that the two terms in the expression for $\Sigma^{\prime}$ are of chiral order $p^{2}$ and $p^{4}$, respectively. That the self-energy of $\mathcal{O}\left(p^{8}\right)$ does not contribute at NNLO is evident from Eq. (64), since the matrix element of the axial current operator itself is of $\mathcal{O}\left(p^{2}\right)$ or higher. By combining all the above results and expressing everything, analogously to Eq. (51), in terms of the lowest order meson mass $M_{0}$, the final result for the decay constant at NNLO is obtained as

$$
\begin{aligned}
F_{\text {phys }} & =F_{0}+\overbrace{F_{4}\left(\chi_{i}\right)+\left.F_{0} \frac{\partial \Sigma_{4}\left(p^{2}, \chi_{i}\right)}{2 \partial p^{2}}\right|_{M_{0}^{2}}}^{\mathcal{O}\left(p^{4}\right) \text { contribution }} \\
& +F_{0} \frac{3}{8}\left(\left.\frac{\partial \Sigma_{4}\left(p^{2}, \chi_{i}\right)}{\partial p^{2}}\right|_{M_{0}^{2}}\right)^{2}+\left.F_{0} \frac{\partial \Sigma_{6}\left(p^{2}, \chi_{i}\right)}{2 \partial p^{2}}\right|_{M_{0}^{2}} \\
& +\left.F_{4}\left(\chi_{i}\right) \frac{\partial \Sigma_{4}\left(p^{2}, \chi_{i}\right)}{2 \partial p^{2}}\right|_{M_{0}^{2}}+F_{6}\left(M_{0}^{2}, \chi_{i}\right)+\mathcal{O}\left(p^{8}\right),
\end{aligned}
$$

where all noncontributing terms have been discarded. In principle, the NLO result indicated by braces in the above equation depends on $M_{\text {phys }}^{2}$ and has to be expanded, analogously to the calculation of the meson masses, around $M_{0}^{2}$ up to $\mathcal{O}\left(p^{6}\right)$. However, the extra terms generated by such an expansion can be eliminated using the identities

$$
\frac{\partial F_{4}\left(p^{2}, \chi_{i}\right)}{\partial p^{2}}=0, \quad \frac{\partial^{2} \Sigma_{4}\left(p^{2}, \chi_{i}\right)}{\partial\left(p^{2}\right)^{2}}=0 .
$$

The former one follows from the fact that the matrix element $F_{4}$ does not depend on $p^{2}$, and the latter one is valid since the highest power of the momentum $p$ that appears in the self-energy $\Sigma_{4}$ is $p^{2}$. The NNLO decay constants of the charged pseudoscalar mesons so obtained depend on the $\mathcal{O}\left(p^{6}\right)$ LEC:s $K_{19}^{r}$ through $K_{23}^{r}$.
The physical decay constant of a charged pseudoscalar meson $\Phi_{i j}$ is given to NNLO in the form

$$
F_{\text {phys }}=F_{0}\left[1+\frac{f^{(4) \mathrm{vs}}}{F_{0}^{2}}+\frac{f_{\mathrm{ct}}^{(6) \mathrm{vs}}+f_{\text {loops }}^{(6) \mathrm{vs}}}{F_{0}^{4}}+\mathcal{O}\left(p^{8}\right)\right] \text {, }
$$

where the $\mathcal{O}\left(p^{4}\right)$ and $\mathcal{O}\left(p^{6}\right)$ contributions have been separated and denoted by $f$ rather than $\delta$ in order to minimize the potential for confusion with the rather similar expressions for the pseudoscalar meson masses. Again, the NNLO contribution $f^{(6)}$ has been further split into the contributions from the chiral loops and from the $\mathcal{O}\left(p^{6}\right)$ counterterms. As for the meson masses, the superscripts (v) and (s) indicate the values of $d_{\text {val }}$ and $d_{\text {sea }}$, respectively. Up to NLO, the pseudoscalar meson decay constant for $d_{\text {val }}=2$ and $d_{\text {sea }}=3$ is given by

$$
\begin{aligned}
f^{(4) 23}= & 12 L_{4}^{r} \bar{\chi}_{1}+4 L_{5}^{r} \chi_{13} \\
& +\bar{A}\left(\chi_{p}\right)\left[1 / 6 R_{q \pi \eta}^{p}-1 / 12 R_{p}^{c}\right] \\
& +1 / 4 \bar{A}\left(\chi_{p s}\right)-1 / 12 \bar{A}\left(\chi_{m}\right) R_{m n 13}^{v} \\
& -1 / 12 \bar{B}\left(\chi_{p}, \chi_{p}, 0\right) R_{p}^{d}
\end{aligned}
$$

and agrees as expected with Refs. 3, 7]. The LEC:s at $\mathcal{O}\left(p^{6}\right)$ give the following contribution to the pseudoscalar meson decay constant for $d_{\text {val }}=2$ and $d_{\text {sea }}=3$,

$$
\begin{aligned}
f_{\mathrm{ct}}^{(6) 23}= & 4 K_{19}^{r} \chi_{p}^{2}+24 K_{20}^{r} \bar{\chi}_{1} \chi_{13}+24 K_{21}^{r} \bar{\chi}_{2} \\
& +72 K_{22}^{r} \bar{\chi}_{1}^{2}+8 K_{23}^{r} \chi_{1} \chi_{3}
\end{aligned}
$$

The expression for the chiral loop contribution is rather long, but it can be brought to a manageable form with the new notation introduced earlier in this paper. The result for $d_{\text {val }}=2$ and $d_{\text {sea }}=3$ is 


$$
\begin{aligned}
& f_{\text {loops }}^{(6) 23}=\pi_{16} L_{0}^{r}\left[4 / 9 \chi_{\pi} \chi_{\eta}-1 / 2 \chi_{1} \chi_{3}+\chi_{13}^{2}-13 / 3 \bar{\chi}_{1} \chi_{13}-35 / 18 \bar{\chi}_{2}\right]-2 \pi_{16} L_{1}^{r} \chi_{13}^{2}-\pi_{16} L_{2}^{r}\left[11 / 3 \chi_{\pi} \chi_{\eta}+\chi_{13}^{2}\right. \\
& \left.+13 / 3 \bar{\chi}_{2}\right]+\pi_{16} L_{3}^{r}\left[4 / 9 \chi_{\pi} \chi_{\eta}-7 / 12 \chi_{1} \chi_{3}+11 / 6 \chi_{13}^{2}-17 / 6 \bar{\chi}_{1} \chi_{13}-43 / 36 \bar{\chi}_{2}\right]+\pi_{16}^{2}\left[-15 / 64 \chi_{\pi} \chi_{\eta}\right. \\
& \left.-59 / 384 \chi_{1} \chi_{3}+65 / 384 \chi_{13}^{2}-1 / 2 \bar{\chi}_{1} \chi_{13}-43 / 128 \bar{\chi}_{2}\right]-48 L_{4}^{r} L_{5}^{r} \bar{\chi}_{1} \chi_{13}-72 L_{4}^{r 2} \bar{\chi}_{1}^{2}-8 L_{5}^{r 2} \chi_{13}^{2} \\
& +\bar{A}\left(\chi_{p}\right) \pi_{16}\left[-1 / 8 \bar{\chi}_{1} R_{q \pi \eta}^{p}+1 / 16 \bar{\chi}_{1} R_{p}^{c}-1 / 12 R_{q \pi \eta}^{p} \chi_{13}+1 / 48 R_{p}^{c} \chi_{q}+1 / 48 R_{p}^{c} \chi_{13}\right] \\
& +\bar{A}\left(\chi_{p}\right) L_{0}^{r}\left[8 / 3 R_{q \pi \eta}^{p} \chi_{p}+2 / 3 R_{p}^{c} \chi_{p}+2 / 3 R_{p}^{d}\right]+\bar{A}\left(\chi_{p}\right) L_{3}^{r}\left[2 / 3 R_{q \pi \eta}^{p} \chi_{p}+5 / 3 R_{p}^{c} \chi_{p}+5 / 3 R_{p}^{d}\right] \\
& -\bar{A}\left(\chi_{p}\right) L_{4}^{r}\left[2 \bar{\chi}_{1} R_{q \pi \eta}^{p}-\bar{\chi}_{1} R_{p}^{c}\right]-\bar{A}\left(\chi_{p}\right) L_{5}^{r}\left[2 / 3 R_{q \pi \eta}^{p} \chi_{13}+1 / 3 R_{p}^{c} \chi_{13}\right]+\bar{A}\left(\chi_{p}\right)^{2}\left[1 / 16+1 / 72\left(R_{q \pi \eta}^{p}\right)^{2}\right. \\
& \left.-1 / 72 R_{q \pi \eta}^{p} R_{p}^{c}+1 / 288\left(R_{p}^{c}\right)^{2}\right]-\bar{A}\left(\chi_{p}\right) \bar{A}\left(\chi_{p s}\right)\left[1 / 36 R_{q \pi \eta}^{p}+5 / 72 R_{s \pi \eta}^{p}-7 / 144 R_{p}^{c}\right] \\
& \text { - } \bar{A}\left(\chi_{p}\right) \bar{A}\left(\chi_{q s}\right)\left[1 / 36 R_{q \pi \eta}^{p}+1 / 24 R_{s \pi \eta}^{p}+1 / 48 R_{p}^{c}\right]-\bar{A}\left(\chi_{p}\right) \bar{A}\left(\chi_{m}\right)\left[1 / 72 R_{q m n}^{p} R_{m n 13}^{v}-1 / 144 R_{p}^{c} R_{m n 13}^{v}\right] \\
& +1 / 8 \bar{A}\left(\chi_{p}\right) \bar{A}\left(\chi_{13}\right)-1 / 108 \bar{A}\left(\chi_{p}\right) \bar{A}\left(\chi_{s t}\right)\left(R_{s \pi \eta}^{p}\right)^{2} R_{t s s p p}^{z}+\bar{A}\left(\chi_{p}\right) \bar{B}\left(\chi_{p}, \chi_{p}, 0\right)\left[1 / 4 \chi_{p}-1 / 18 R_{q \pi \eta}^{p} R_{p}^{c} \chi_{p}\right. \\
& \left.-1 / 72 R_{q \pi \eta}^{p} R_{p}^{d}+1 / 18\left(R_{p}^{c}\right)^{2} \chi_{p}+1 / 144 R_{p}^{c} R_{p}^{d}\right]+1 / 18 \bar{A}\left(\chi_{p}\right) \bar{B}\left(\chi_{p}, \chi_{m}, 0\right) R_{q m p}^{z} R_{p}^{c} R_{m n 13}^{v} \chi_{p} \\
& \text { - } \bar{A}\left(\chi_{p}\right) \bar{B}\left(\chi_{q}, \chi_{q}, 0\right)\left[1 / 72 R_{q \pi \eta}^{p} R_{q}^{d}-1 / 144 R_{p}^{c} R_{q}^{d}\right]-1 / 12 \bar{A}\left(\chi_{p}\right) \bar{B}\left(\chi_{p s}, \chi_{p s}, 0\right) R_{s \pi \eta}^{p} \chi_{p s} \\
& -1 / 18 \bar{A}\left(\chi_{p}\right) \bar{B}\left(\chi_{1}, \chi_{3}, 0\right) R_{p \pi \eta}^{q} R_{p}^{c} \chi_{p}+1 / 18 \bar{A}\left(\chi_{p}\right) \bar{C}\left(\chi_{p}, \chi_{p}, \chi_{p}, 0\right) R_{p}^{c} R_{p}^{d} \chi_{p}+\bar{A}\left(\chi_{p}, \varepsilon\right) \pi_{16}\left[1 / 8 \bar{\chi}_{1} R_{q \pi \eta}^{p}\right. \\
& \left.-1 / 16 \bar{\chi}_{1} R_{p}^{c}-1 / 16 R_{p}^{c} \chi_{p}-1 / 16 R_{p}^{d}\right]+\bar{A}\left(\chi_{p s}\right) \pi_{16}\left[1 / 16 \chi_{p s}-3 / 16 \chi_{q s}-3 / 16 \bar{\chi}_{1}\right]-2 \bar{A}\left(\chi_{p s}\right) L_{0}^{r} \chi_{p s} \\
& -5 \bar{A}\left(\chi_{p s}\right) L_{3}^{r} \chi_{p s}-3 \bar{A}\left(\chi_{p s}\right) L_{4}^{r} \bar{\chi}_{1}+\bar{A}\left(\chi_{p s}\right) L_{5}^{r} \chi_{13}-1 / 32 \bar{A}\left(\chi_{p s}\right)^{2}-1 / 16 \bar{A}\left(\chi_{p s}\right) \bar{A}\left(\chi_{p t}\right) \\
& +1 / 16 \bar{A}\left(\chi_{p s}\right) \bar{A}\left(\chi_{q t}\right)+\bar{A}\left(\chi_{p s}\right) \bar{A}\left(\chi_{m}\right)\left[7 / 144 R_{n p p}^{m}-5 / 72 R_{n p s}^{m}-1 / 48 R_{n q q}^{m}+5 / 72 R_{n q s}^{m}-1 / 36 R_{n 13}^{m}\right] \\
& +\bar{A}\left(\chi_{p s}\right) \bar{B}\left(\chi_{p}, \chi_{p}, 0\right)\left[1 / 24 R_{s \pi \eta}^{p} \chi_{p}-5 / 24 R_{s \pi \eta}^{p} \chi_{p s}\right]-\bar{A}\left(\chi_{p s}\right) \bar{B}\left(\chi_{p}, \chi_{m}, 0\right)\left[1 / 18 R_{n p s}^{m} R_{q p m}^{z} \chi_{p}\right. \\
& \left.+1 / 9 R_{n p s}^{m} R_{q p m}^{z} \chi_{p s}\right]-1 / 48 \bar{A}\left(\chi_{p s}\right) \bar{B}\left(\chi_{q}, \chi_{q}, 0\right) R_{q}^{d}+1 / 18 \bar{A}\left(\chi_{p s}\right) \bar{B}\left(\chi_{1}, \chi_{3}, 0\right) R_{s \pi \eta}^{q} \chi_{s} \\
& +1 / 9 \bar{A}\left(\chi_{p s}\right) \bar{B}\left(\chi_{1}, \chi_{3}, 0, k\right) R_{s \pi \eta}^{q}+3 / 16 \bar{A}\left(\chi_{p s}, \varepsilon\right) \pi_{16}\left[\chi_{s}+\bar{\chi}_{1}\right]+\bar{A}\left(\chi_{m}\right) \pi_{16}\left[1 / 16 \bar{\chi}_{1} R_{m n 13}^{v}\right. \\
& \left.-1 / 48 R_{m n 13}^{v} \chi_{m}+1 / 16 R_{m n 13}^{v} \chi_{13}\right]+\bar{A}\left(\chi_{m}\right) L_{0}^{r}\left[4 R_{n 13}^{m} \chi_{m}+2 / 3 R_{m n 13}^{v} \chi_{m}\right]-8 \bar{A}\left(\chi_{m}\right) L_{1}^{r} \chi_{m} \\
& -2 \bar{A}\left(\chi_{m}\right) L_{2}^{r} \chi_{m}+\bar{A}\left(\chi_{m}\right) L_{3}^{r}\left[4 R_{n 13}^{m} \chi_{m}+5 / 3 R_{m n 13}^{v} \chi_{m}\right]+\bar{A}\left(\chi_{m}\right) L_{4}^{r}\left[4 \chi_{m}+\bar{\chi}_{1} R_{m n 13}^{v}\right] \\
& -\bar{A}\left(\chi_{m}\right) L_{5}^{r}\left[4 / 3 R_{n 13}^{m} \chi_{13}+1 / 3 R_{m n 13}^{v} \chi_{13}\right]+1 / 288 \bar{A}\left(\chi_{m}\right)^{2}\left(R_{m n 13}^{v}\right)^{2}-1 / 108 \bar{A}\left(\chi_{m}\right) \bar{A}\left(\chi_{s t}\right) R_{m n 13}^{v} R_{m n s t}^{v} \\
& +\bar{A}\left(\chi_{m}\right) \bar{B}\left(\chi_{p}, \chi_{p}, 0\right)\left[-1 / 18 R_{q m n}^{p} R_{n p p}^{m} \chi_{p}-1 / 108\left(R_{s m n}^{p}\right)^{2} R_{n s s}^{m} \chi_{s}+1 / 18 R_{n p p}^{m} R_{p}^{c} \chi_{p}+1 / 144 R_{p}^{d} R_{m n 13}^{v}\right] \\
& +\bar{A}\left(\chi_{m}\right) \bar{B}\left(\chi_{p}, \chi_{m}, 0\right)\left[-1 / 54 R_{s m n}^{p} R_{n p s}^{m} R_{n s s}^{m} \chi_{s}+1 / 54 R_{s m n}^{p} R_{n q s}^{m} R_{n s s}^{m} \chi_{s}+1 / 18\left(R_{n p p}^{m}\right)^{2} \chi_{p}\right. \\
& \left.-1 / 18 R_{n p p}^{m} R_{n 13}^{m} \chi_{p}\right]+\bar{A}\left(\chi_{m}\right) \bar{B}\left(\chi_{p}, \chi_{n}, 0\right)\left[-1 / 54 R_{s m n}^{p} R_{n s s}^{m} R_{m p s}^{n} \chi_{s}+1 / 54 R_{s m n}^{p} R_{n s s}^{m} R_{m q s}^{n} \chi_{s}\right. \\
& \left.+1 / 18 R_{n p p}^{m} R_{m p p}^{n} \chi_{p}-1 / 18 R_{n p p}^{m} R_{m 13}^{n} \chi_{p}\right]-1 / 12 \bar{A}\left(\chi_{m}\right) \bar{B}\left(\chi_{p s}, \chi_{p s}, 0\right) R_{n p s}^{m} \chi_{p s} \\
& \text { - 1/108 } \bar{A}\left(\chi_{m}\right) \bar{B}\left(\chi_{m}, \chi_{m}, 0\right)\left(R_{n s s}^{m}\right)^{2} R_{m n 13}^{v} \chi_{s}-1 / 54 \bar{A}\left(\chi_{m}\right) \bar{B}\left(\chi_{m}, \chi_{n}, 0\right)\left[R_{n p s}^{m} R_{n s s}^{m} R_{m p s}^{n} \chi_{s}\right. \\
& \text { - } \left.R_{n q s}^{m} R_{n s s}^{m} R_{m p s}^{n} \chi_{s}\right]-1 / 108 \bar{A}\left(\chi_{m}\right) \bar{B}\left(\chi_{n}, \chi_{n}, 0\right) R_{n s s}^{m} R_{m s s}^{n} R_{n m 13}^{v} \chi_{s} \\
& \text { - } \bar{A}\left(\chi_{m}\right) \bar{B}\left(\chi_{1}, \chi_{3}, 0\right)\left[1 / 18 R_{p m n}^{q} R_{n p p}^{m} \chi_{p}-1 / 54 R_{n s s}^{m} R_{s m n}^{1} R_{s m n}^{3} \chi_{s}\right] \\
& +1 / 18 \bar{A}\left(\chi_{m}\right) \bar{C}\left(\chi_{p}, \chi_{p}, \chi_{p}, 0\right) R_{n p p}^{m} R_{p}^{d} \chi_{p}+\bar{A}\left(\chi_{m}, \varepsilon\right) \pi_{16}\left[1 / 8 \chi_{m}-1 / 16 \bar{\chi}_{1} R_{m n 13}^{v}-1 / 8 R_{n 13}^{m} \chi_{m}\right. \\
& \left.-1 / 16 R_{m n 13}^{v} \chi_{m}\right]+1 / 144 \bar{A}\left(\chi_{\pi}\right) \bar{A}\left(\chi_{\eta}\right) R_{\pi \eta 13}^{v} R_{\eta \pi 13}^{v}+\bar{A}\left(\chi_{1}\right) \bar{A}\left(\chi_{3}\right)\left[-1 / 72 R_{q \pi \eta}^{p} R_{q}^{c}+1 / 36 R_{3 \pi \eta}^{1} R_{1 \pi \eta}^{3}\right. \\
& \left.+1 / 144 R_{1}^{c} R_{3}^{c}\right]-4 \bar{A}\left(\chi_{13}\right) L_{1}^{r} \chi_{13}-10 \bar{A}\left(\chi_{13}\right) L_{2}^{r} \chi_{13}+1 / 8 \bar{A}\left(\chi_{13}\right)^{2}-1 / 2 \bar{A}\left(\chi_{13}\right) \bar{B}\left(\chi_{1}, \chi_{3}, 0, k\right) \\
& +1 / 4 \bar{A}\left(\chi_{13}, \varepsilon\right) \pi_{16} \chi_{13}+1 / 16 \bar{A}\left(\chi_{1 s}\right) \bar{A}\left(\chi_{3 s}\right)-16 \bar{A}\left(\chi_{s t}\right) L_{1}^{r} \chi_{s t}-4 \bar{A}\left(\chi_{s t}\right) L_{2}^{r} \chi_{s t}+8 \bar{A}\left(\chi_{s t}\right) L_{4}^{r} \chi_{s t} \\
& +1 / 18 \bar{A}\left(\chi_{s t}\right) \bar{B}\left(\chi_{p}, \chi_{p}, 0\right) R_{s \pi \eta}^{p} R_{t \pi \eta}^{p} \chi_{s t}+1 / 54 \bar{A}\left(\chi_{s t}\right) \bar{B}\left(\chi_{p}, \chi_{m}, 0\right)\left[R_{p p s t}^{w m}-R_{p q s t}^{w m}\right] \\
& -1 / 54 \bar{A}\left(\chi_{s t}\right) \bar{B}\left(\chi_{p}, \chi_{m}, 0, k\right)\left[R_{p p s t}^{u m}-R_{p q s t}^{u m}\right]+1 / 18 \bar{A}\left(\chi_{s t}\right) \bar{B}\left(\chi_{m}, \chi_{m}, 0\right) R_{n s t}^{m} R_{m n 13}^{v} \chi_{s t} \\
& +1 / 54 \bar{A}\left(\chi_{s t}\right) \bar{B}\left(\chi_{\pi}, \chi_{\eta}, 0\right)\left[R_{p p s t}^{w \pi \eta}-R_{p q s t}^{w \pi \eta}\right]-1 / 54 \bar{A}\left(\chi_{s t}\right) \bar{B}\left(\chi_{\pi}, \chi_{\eta}, 0, k\right)\left[R_{p p s t}^{u \pi \eta}-R_{p q s t}^{u \pi \eta}\right] \\
& -1 / 54 \bar{A}\left(\chi_{s t}\right) \bar{B}\left(\chi_{1}, \chi_{3}, 0\right) R_{13 s t}^{w}+1 / 54 \bar{A}\left(\chi_{s t}\right) \bar{B}\left(\chi_{1}, \chi_{3}, 0, k\right) R_{13 s t}^{u}+1 / 4 \bar{A}\left(\chi_{s t}, \varepsilon\right) \pi_{16} \chi_{s t} \\
& +\bar{B}\left(\chi_{p}, \chi_{p}, 0\right) \pi_{16}\left[1 / 16 \bar{\chi}_{1} R_{p}^{d}+1 / 96 R_{p}^{d} \chi_{p}+1 / 32 R_{p}^{d} \chi_{q}\right]+2 / 3 \bar{B}\left(\chi_{p}, \chi_{p}, 0\right) L_{0}^{r} R_{p}^{d} \chi_{p} \\
& +5 / 3 \bar{B}\left(\chi_{p}, \chi_{p}, 0\right) L_{3}^{r} R_{p}^{d} \chi_{p}+\bar{B}\left(\chi_{p}, \chi_{p}, 0\right) L_{4}^{r}\left[-4 \bar{\chi}_{1} R_{q \pi \eta}^{p} \chi_{p}+2 \bar{\chi}_{1} R_{p}^{c} \chi_{p}+3 \bar{\chi}_{1} R_{p}^{d}\right] \\
& +\bar{B}\left(\chi_{p}, \chi_{p}, 0\right) L_{5}^{r}\left[-4 / 3 R_{q \pi \eta}^{p} \chi_{1} \chi_{3}+2 / 3 R_{p}^{c} \chi_{p}^{2}-1 / 3 R_{p}^{d} \chi_{13}\right]+\bar{B}\left(\chi_{p}, \chi_{p}, 0\right) L_{6}^{r}\left[8 \bar{\chi}_{1} R_{q \pi \eta}^{p} \chi_{p}-4 \bar{\chi}_{1} R_{p}^{c} \chi_{p}\right. \\
& \left.-4 \bar{\chi}_{1} R_{p}^{d}\right]+4 \bar{B}\left(\chi_{p}, \chi_{p}, 0\right) L_{7}^{r}\left(R_{p}^{d}\right)^{2}+\bar{B}\left(\chi_{p}, \chi_{p}, 0\right) L_{8}^{r}\left[8 / 3 R_{q \pi \eta}^{p} \chi_{1} \chi_{3}-4 / 3 R_{p}^{c} \chi_{p}^{2}+4 / 3\left(R_{p}^{d}\right)^{2}\right]
\end{aligned}
$$


$+\bar{B}\left(\chi_{p}, \chi_{p}, 0\right)^{2}\left[-1 / 18 R_{q \pi \eta}^{p} R_{p}^{d} \chi_{p}+1 / 18 R_{p}^{c} R_{p}^{d} \chi_{p}+1 / 288\left(R_{p}^{d}\right)^{2}\right]$

$+1 / 18 \bar{B}\left(\chi_{p}, \chi_{p}, 0\right) \bar{B}\left(\chi_{p}, \chi_{m}, 0\right) R_{q m p}^{z} R_{p}^{d} R_{m n 13}^{v} \chi_{p}-1 / 18 \bar{B}\left(\chi_{p}, \chi_{p}, 0\right) \bar{B}\left(\chi_{1}, \chi_{3}, 0\right) R_{p \pi \eta}^{q} R_{p}^{d} \chi_{p}$

$+1 / 18 \bar{B}\left(\chi_{p}, \chi_{p}, 0\right) \bar{C}\left(\chi_{p}, \chi_{p}, \chi_{p}, 0\right)\left(R_{p}^{d}\right)^{2} \chi_{p}-1 / 16 \bar{B}\left(\chi_{p}, \chi_{p}, 0, \varepsilon\right) \pi_{16}\left[\bar{\chi}_{1} R_{p}^{d}+R_{p}^{d} \chi_{p}\right]$

$+8 \bar{B}\left(\chi_{p}, \chi_{m}, 0\right) L_{7}^{r} R_{q p m}^{z} R_{p}^{d} R_{m 456 n p}^{z}+8 / 3 \bar{B}\left(\chi_{p}, \chi_{m}, 0\right) L_{8}^{r} R_{q p m}^{z} R_{p}^{d} R_{m 456 n p}^{z}-6 \bar{B}\left(\chi_{p s}, \chi_{p s}, 0\right) L_{4}^{r} \bar{\chi}_{1} \chi_{p s}$

$-2 \bar{B}\left(\chi_{p s}, \chi_{p s}, 0\right) L_{5}^{r} \chi_{p s}^{2}+12 \bar{B}\left(\chi_{p s}, \chi_{p s}, 0\right) L_{6}^{r} \bar{\chi}_{1} \chi_{p s}+4 \bar{B}\left(\chi_{p s}, \chi_{p s}, 0\right) L_{8}^{r} \chi_{p s}^{2}$

$+2 \bar{B}\left(\chi_{m}, \chi_{m}, 0\right) L_{4}^{r} \bar{\chi}_{1} R_{m n 13}^{v} \chi_{m}+2 / 3 \bar{B}\left(\chi_{m}, \chi_{m}, 0\right) L_{5}^{r} R_{m n 13}^{v} \chi_{m}^{2}-4 \bar{B}\left(\chi_{m}, \chi_{m}, 0\right) L_{6}^{r}, \bar{\chi}_{1} R_{m n 13}^{v} \chi_{m}$

$+4 \bar{B}\left(\chi_{m}, \chi_{m}, 0\right) L_{7}^{r} R_{311 m m}^{z}\left(R_{m 456 n 1}^{z}\right)^{2}+4 / 3 \bar{B}\left(\chi_{m}, \chi_{m}, 0\right) L_{8}^{r}\left[R_{311 m m}^{z}\left(R_{m 456 n 1}^{z}\right)^{2}-R_{m n 13}^{v} \chi_{m}^{2}\right]$

$+8 \bar{B}\left(\chi_{\pi}, \chi_{\eta}, 0\right) L_{7}^{r} R_{311 \pi \eta}^{z} R_{\pi 456 \eta 1}^{z} R_{\eta 456 \pi 1}^{z}+8 / 3 \bar{B}\left(\chi_{\pi}, \chi_{\eta}, 0\right) L_{8}^{r} R_{311 \pi \eta}^{z} R_{\pi 456 \eta 1}^{z} R_{\eta 456 \pi 1}^{z}$

$+1 / 144 \bar{B}\left(\chi_{1}, \chi_{1}, 0\right) \bar{B}\left(\chi_{3}, \chi_{3}, 0\right) R_{1}^{d} R_{3}^{d}-8 \bar{B}\left(\chi_{1}, \chi_{3}, 0\right) L_{7}^{r} R_{1}^{d} R_{3}^{d}-8 / 3 \bar{B}\left(\chi_{1}, \chi_{3}, 0\right) L_{8}^{r} R_{1}^{d} R_{3}^{d}$

$+4 \bar{C}\left(\chi_{p}, \chi_{p}, \chi_{p}, 0\right) L_{4}^{r} \bar{\chi}_{1} R_{p}^{d} \chi_{p}+4 / 3 \bar{C}\left(\chi_{p}, \chi_{p}, \chi_{p}, 0\right) L_{5}^{r} R_{p}^{d} \chi_{p}^{2}-8 \bar{C}\left(\chi_{p}, \chi_{p}, \chi_{p}, 0\right) L_{6}^{r} \bar{\chi}_{1} R_{p}^{d} \chi_{p}$

$-8 / 3 \bar{C}\left(\chi_{p}, \chi_{p}, \chi_{p}, 0\right) L_{8}^{r} R_{p}^{d} \chi_{p}^{2}+H^{F}\left(1, \chi_{p}, \chi_{p}, \chi_{13}, \chi_{13}\right)\left[1 / 8 \chi_{p}-1 / 16 \chi_{13}-1 / 36\left(R_{q \pi \eta}^{p}\right)^{2} \chi_{13}\right.$

$\left.+1 / 36 R_{q \pi \eta}^{p} R_{p}^{c} \chi_{13}-1 / 144\left(R_{p}^{c}\right)^{2} \chi_{13}\right]+H^{F}\left(1, \chi_{p}, \chi_{1 s}, \chi_{3 s}, \chi_{13}\right)\left[-1 / 12 R_{q \pi \eta}^{p} \chi_{q s}+1 / 24 R_{q \pi \eta}^{p} \chi_{13}\right.$

$\left.-1 / 16 R_{s \pi \eta}^{p} \chi_{p}+1 / 48 R_{s \pi \eta}^{p} \chi_{q}+1 / 24 R_{p}^{c} \chi_{p s}\right]-1 / 8 H^{F}\left(1, \chi_{p s}, \chi_{q t}, \chi_{s t}, \chi_{13}\right) \chi_{s t}$

$+H^{F}\left(1, \chi_{m}, \chi_{p}, \chi_{13}, \chi_{13}\right)\left[1 / 36 R_{q m n}^{p} R_{m n 13}^{v} \chi_{13}-1 / 72 R_{p}^{c} R_{m n 13}^{v} \chi_{13}\right]$

$-1 / 144 H^{F}\left(1, \chi_{m}, \chi_{m}, \chi_{13}, \chi_{13}\right)\left(R_{m n 13}^{v}\right)^{2} \chi_{13}+1 / 48 H^{F}\left(1, \chi_{m}, \chi_{1 s}, \chi_{3 s}, \chi_{13}\right)\left[-R_{n p s}^{m} \chi_{p}+R_{n p s}^{m} \chi_{q}\right.$

$\left.+R_{m n p s}^{v} \chi_{m}+R_{m n 13}^{v} \chi_{s}\right]-1 / 72 H^{F}\left(1, \chi_{\pi}, \chi_{\eta}, \chi_{13}, \chi_{13}\right) R_{\pi \eta 13}^{v} R_{\eta \pi 13}^{v} \chi_{13}$

$+H^{F}\left(1, \chi_{1}, \chi_{13}, \chi_{3}, \chi_{13}\right)\left[1 / 36 R_{q \pi \eta}^{p} R_{q}^{c} \chi_{13}-1 / 18 R_{3 \pi \eta}^{1} R_{1 \pi \eta}^{3} \chi_{13}-1 / 72 R_{1}^{c} R_{3}^{c} \chi_{13}\right]$

$-1 / 8 H^{F}\left(1, \chi_{13}, \chi_{13}, \chi_{13}, \chi_{13}\right) \chi_{13}+H^{F}\left(2, \chi_{p}, \chi_{p}, \chi_{13}, \chi_{13}\right)\left[1 / 36 R_{q \pi \eta}^{p} R_{p}^{d} \chi_{13}-1 / 72 R_{p}^{c} R_{p}^{d} \chi_{13}\right]$

$-1 / 72 H^{F}\left(2, \chi_{p}, \chi_{m}, \chi_{13}, \chi_{13}\right) R_{p}^{d} R_{m n 13}^{v} \chi_{13}+H^{F}\left(2, \chi_{p}, \chi_{13}, \chi_{q}, \chi_{13}\right)\left[1 / 36 R_{p \pi \eta}^{q} R_{p}^{d} \chi_{13}-1 / 72 R_{q}^{c} R_{p}^{d} \chi_{13}\right]$

$+1 / 24 H^{F}\left(2, \chi_{p}, \chi_{1 s}, \chi_{3 s}, \chi_{13}\right) R_{p}^{d} \chi_{p s}-1 / 144 H^{F}\left(5, \chi_{p}, \chi_{p}, \chi_{13}, \chi_{13}\right)\left(R_{p}^{d}\right)^{2} \chi_{13}$

$-1 / 72 H^{F}\left(5, \chi_{1}, \chi_{3}, \chi_{13}, \chi_{13}\right) R_{1}^{d} R_{3}^{d} \chi_{13}+H^{F^{\prime}}\left(1, \chi_{p}, \chi_{p}, \chi_{13}, \chi_{13}\right)\left[-1 / 8 \chi_{p} \chi_{13}-3 / 16 \chi_{13}^{2}\right.$

$\left.-1 / 36\left(R_{q \pi \eta}^{p}\right)^{2} \chi_{13}^{2}+1 / 12 R_{q \pi \eta}^{p} R_{p}^{c} \chi_{13}^{2}-1 / 48\left(R_{p}^{c}\right)^{2} \chi_{13}^{2}\right]+H^{F^{\prime}}\left(1, \chi_{p}, \chi_{1 s}, \chi_{3 s}, \chi_{13}\right)\left[-5 / 24 R_{q \pi \eta}^{p} \chi_{q} \chi_{13}\right.$

$\left.+1 / 12 R_{q \pi \eta}^{p} \chi_{p s} \chi_{13}+1 / 16 R_{s \pi \eta}^{p} \chi_{q} \chi_{13}+7 / 48 R_{s \pi \eta}^{p} \chi_{13} \chi_{s}-1 / 24 R_{p}^{c} \chi_{p s} \chi_{13}\right]$

$+1 / 8 H^{F^{\prime}}\left(1, \chi_{p s}, \chi_{q t}, \chi_{s t}, \chi_{13}\right) \chi_{13} \chi_{s t}+H^{F^{\prime}}\left(1, \chi_{m}, \chi_{p}, \chi_{13}, \chi_{13}\right)\left[1 / 9 R_{q m n}^{p} R_{n 13}^{m} \chi_{13}^{2}\right.$

$\left.-1 / 36 R_{q m n}^{p} R_{m n 13}^{v} \chi_{13}^{2}+1 / 18 R_{n p p}^{m} R_{p}^{c} \chi_{13}^{2}+1 / 72 R_{p}^{c} R_{m n 13}^{v} \chi_{13}^{2}\right]$

$+H^{F^{\prime}}\left(1, \chi_{m}, \chi_{m}, \chi_{13}, \chi_{13}\right)\left[1 / 9\left(R_{n 13}^{m}\right)^{2} \chi_{13}^{2}+1 / 9 R_{n 13}^{m} R_{m n 13}^{v} \chi_{13}^{2}+5 / 144\left(R_{m n 13}^{v}\right)^{2} \chi_{13}^{2}\right]$

$+H^{F^{\prime}}\left(1, \chi_{m}, \chi_{1 s}, \chi_{3 s}, \chi_{13}\right)\left[-1 / 16 R_{n p s}^{m} \chi_{p} \chi_{13}-5 / 48 R_{n p s}^{m} \chi_{q} \chi_{13}+1 / 6 R_{n 13}^{m} \chi_{13}^{2}-1 / 48 R_{m n p s}^{v} \chi_{m} \chi_{13}\right.$

$\left.-1 / 48 R_{m n 13}^{v} \chi_{13} \chi_{s}\right]+H^{F^{\prime}}\left(1, \chi_{\pi}, \chi_{\eta}, \chi_{13}, \chi_{13}\right)\left[1 / 18 R_{\eta p p}^{\pi} R_{\pi p p}^{\eta} \chi_{13}^{2}+1 / 9 R_{\eta 13}^{\pi} R_{\pi 13}^{\eta} \chi_{13}^{2}\right.$

$\left.+1 / 72 R_{\pi \eta 13}^{v} R_{\eta \pi 13}^{v}, \chi_{13}^{2}\right]+H^{F^{\prime}}\left(1, \chi_{1}, \chi_{13}, \chi_{3}, \chi_{13}\right)\left[-1 / 36 R_{q \pi \eta}^{p} R_{q}^{c} \chi_{13}^{2}+1 / 6 R_{3 \pi \eta}^{1} R_{1 \pi \eta}^{3} \chi_{13}^{2}\right.$

$\left.+1 / 72 R_{1}^{c} R_{3}^{c} \chi_{13}^{2}\right]+1 / 8 H^{F^{\prime}}\left(1, \chi_{13}, \chi_{13}, \chi_{13}, \chi_{13}\right) \chi_{13}^{2}-H^{F^{\prime}}\left(2, \chi_{p}, \chi_{p}, \chi_{13}, \chi_{13}\right)\left[1 / 36 R_{q \pi \eta}^{p} R_{p}^{d} \chi_{13}^{2}\right.$

$\left.-5 / 72 R_{p}^{c} R_{p}^{d} \chi_{13}^{2}\right]+H^{F^{\prime}}\left(2, \chi_{p}, \chi_{m}, \chi_{13}, \chi_{13}\right)\left[1 / 18 R_{n p p}^{m} R_{p}^{d} \chi_{13}^{2}+1 / 72 R_{p}^{d} R_{m n 13}^{v} \chi_{13}^{2}\right]$

$-H^{F^{\prime}}\left(2, \chi_{p}, \chi_{13}, \chi_{q}, \chi_{13}\right)\left[1 / 36 R_{p \pi \eta}^{q} R_{p}^{d} \chi_{13}^{2}-1 / 72 R_{q}^{c} R_{p}^{d} \chi_{13}^{2}\right]-1 / 24 H^{F^{\prime}}\left(2, \chi_{p}, \chi_{1 s}, \chi_{3 s}, \chi_{13}\right) R_{p}^{d} \chi_{p s} \chi_{13}$

$+5 / 144 H^{F^{\prime}}\left(5, \chi_{p}, \chi_{p}, \chi_{13}, \chi_{13}\right)\left(R_{p}^{d}\right)^{2} \chi_{13}^{2}+1 / 72 H^{F^{\prime}}\left(5, \chi_{1}, \chi_{3}, \chi_{13}, \chi_{13}\right) R_{1}^{d} R_{3}^{d} \chi_{13}^{2}$

$+H_{1}^{F^{\prime}}\left(1, \chi_{p}, \chi_{p}, \chi_{13}, \chi_{13}\right)\left[\chi_{13}^{2}+2 / 9\left(R_{q \pi \eta}^{p}\right)^{2} \chi_{13}^{2}-2 / 9 R_{q \pi \eta}^{p} R_{p}^{c} \chi_{13}^{2}+1 / 9\left(R_{p}^{c}\right)^{2} \chi_{13}^{2}\right]$

$+1 / 3 H_{1}^{F^{\prime}}\left(1, \chi_{p}, \chi_{1 s}, \chi_{3 s}, \chi_{13}\right) R_{q \pi \eta}^{p} R_{s q p}^{z} \chi_{13}^{2}-1 / 3 H_{1}^{F^{\prime}}\left(1, \chi_{p s}, \chi_{q s}, \chi_{p}, \chi_{13}\right) R_{s \pi \eta}^{p} \chi_{13}^{2}$

$-1 / 3 H_{1}^{F^{\prime}}\left(1, \chi_{p s}, \chi_{q s}, \chi_{m}, \chi_{13}\right) R_{n 13}^{m} R_{s p m}^{z} \chi_{13}^{2}+1 / 9 H_{1}^{F^{\prime}}\left(1, \chi_{13}, \chi_{p}, \chi_{m}, \chi_{13}\right)\left[R_{q m n}^{p} R_{m n 13}^{v} \chi_{13}^{2}\right.$

- $\left.R_{n p p}^{m} R_{q p m}^{z} R_{p}^{c} \chi_{13}^{2}\right]-H_{1}^{F^{\prime}}\left(1, \chi_{13}, \chi_{m}, \chi_{m}, \chi_{13}\right)\left[1 / 9 R_{n 13}^{m} R_{m n 13}^{v} \chi_{13}^{2}+1 / 18\left(R_{m n 13}^{v}\right)^{2} \chi_{13}^{2}\right]$

$-1 / 9 H_{1}^{F^{\prime}}\left(1, \chi_{13}, \chi_{\pi}, \chi_{\eta}, \chi_{13}\right)\left[R_{\eta p p}^{\pi} R_{q \eta p}^{z} R_{\eta \pi 13}^{v} \chi_{13}^{2}-R_{\eta 13}^{\pi} R_{\eta \pi 13}^{v} \chi_{13}^{2}\right]$

$+H_{1}^{F^{\prime}}\left(1, \chi_{13}, \chi_{1}, \chi_{3}, \chi_{13}\right)\left[1 / 9 R_{q \pi \eta}^{p} R_{q}^{c} \chi_{13}^{2}-2 / 9 R_{3 \pi \eta}^{1} R_{1 \pi \eta}^{3} \chi_{13}^{2}\right]$ 


$$
\begin{aligned}
& +1 / 9 H_{1}^{F^{\prime}}\left(3, \chi_{13}, \chi_{p}, \chi_{p}, \chi_{13}\right)\left[R_{q \pi \eta}^{p} R_{p}^{d} \chi_{13}^{2}-R_{p}^{c} R_{p}^{d} \chi_{13}^{2}\right]+1 / 9 H_{1}^{F^{\prime}}\left(3, \chi_{13}, \chi_{p}, \chi_{q}, \chi_{13}\right) R_{p \pi \eta}^{q} R_{p}^{d} \chi_{13}^{2} \\
& +1 / 9 H_{1}^{F^{\prime}}\left(3, \chi_{13}, \chi_{p}, \chi_{m}, \chi_{13}\right) R_{n 13}^{m} R_{p q m}^{z} R_{p}^{d} \chi_{13}^{2}-1 / 18 H_{1}^{F^{\prime}}\left(7, \chi_{13}, \chi_{p}, \chi_{p}, \chi_{13}\right)\left(R_{p}^{d}\right)^{2} \chi_{13}^{2} \\
& -3 / 8 H_{21}^{F^{\prime}}\left(1, \chi_{p}, \chi_{p}, \chi_{13}, \chi_{13}\right) \chi_{13}^{2}-1 / 8 H_{21}^{F^{\prime}}\left(1, \chi_{p}, \chi_{1 s}, \chi_{3 s}, \chi_{13}\right) R_{q \pi \eta}^{p} R_{s q p}^{z} \chi_{13}^{2} \\
& +1 / 8 H_{21}^{F^{\prime}}\left(1, \chi_{p s}, \chi_{q s}, \chi_{p}, \chi_{13}\right)\left[R_{q \pi \eta}^{p} \chi_{13}^{2}+R_{s \pi \eta}^{p} \chi_{13}^{2}-R_{p}^{c} \chi_{13}^{2}\right]+1 / 8 H_{21}^{F^{\prime}}\left(1, \chi_{p s}, \chi_{q s}, \chi_{q}, \chi_{13}\right) R_{p \pi \eta}^{q} R_{s p q}^{z} \chi_{13}^{2} \\
& +1 / 8 H_{21}^{F^{\prime}}\left(1, \chi_{p s}, \chi_{q s}, \chi_{m}, \chi_{13}\right) R_{n 13}^{m} R_{p q m}^{z} R_{s p m}^{z} \chi_{13}^{2}-1 / 8 H_{21}^{F^{\prime}}\left(1, \chi_{m}, \chi_{1 s}, \chi_{3 s}, \chi_{13}\right) R_{n 13}^{m} R_{s 1 m}^{z} R_{s 3 m}^{z} \chi_{13}^{2} \\
& +H_{21}^{F^{\prime}}\left(1, \chi_{13}, \chi_{p}, \chi_{p}, \chi_{13}\right)\left[3 / 16 \chi_{13}^{2}+1 / 12\left(R_{q \pi \eta}^{p}\right)^{2} \chi_{13}^{2}-1 / 12 R_{q \pi \eta}^{p} R_{p}^{c} \chi_{13}^{2}+1 / 48\left(R_{p}^{c}\right)^{2} \chi_{13}^{2}\right] \\
& -H_{21}^{F^{\prime}}\left(1, \chi_{13}, \chi_{p}, \chi_{m}, \chi_{13}\right)\left[1 / 12 R_{q m n}^{p} R_{m n 13}^{v} \chi_{13}^{2}-1 / 24 R_{p}^{c} R_{m n 13}^{v} \chi_{13}^{2}\right] \\
& +1 / 48 H_{21}^{F^{\prime}}\left(1, \chi_{13}, \chi_{m}, \chi_{m}, \chi_{13}\right)\left(R_{m n 13}^{v}\right)^{2} \chi_{13}^{2}+1 / 24 H_{21}^{F^{\prime}}\left(1, \chi_{13}, \chi_{\pi}, \chi_{\eta}, \chi_{13}\right) R_{\pi \eta 13}^{v} R_{\eta \pi 13}^{v} \chi_{13}^{2} \\
& +H_{21}^{F^{\prime}}\left(1, \chi_{13}, \chi_{1}, \chi_{3}, \chi_{13}\right)\left[-1 / 12 R_{q \pi \eta}^{p} R_{q}^{c} \chi_{13}^{2}+1 / 6 R_{3 \pi \eta}^{1} R_{1 \pi \eta}^{3} \chi_{13}^{2}+1 / 24 R_{1}^{c} R_{3}^{c} \chi_{13}^{2}\right] \\
& +3 / 8 H_{21}^{F^{\prime}}\left(1, \chi_{13}, \chi_{13}, \chi_{13}, \chi_{13}\right) \chi_{13}^{2}+3 / 8 H_{21}^{F^{\prime}}\left(1, \chi_{s t}, \chi_{p s}, \chi_{q t}, \chi_{13}\right) \chi_{13}^{2} \\
& -1 / 8 H_{21}^{F^{\prime}}\left(3, \chi_{p s}, \chi_{p}, \chi_{q s}, \chi_{13}\right) R_{p}^{d} \chi_{13}^{2}-H_{21}^{F^{\prime}}\left(3, \chi_{13}, \chi_{p}, \chi_{p}, \chi_{13}\right)\left[1 / 12 R_{q \pi \eta}^{p} R_{p}^{d} \chi_{13}^{2}-1 / 24 R_{p}^{c} R_{p}^{d} \chi_{13}^{2}\right] \\
& -H_{21}^{F^{\prime}}\left(3, \chi_{13}, \chi_{p}, \chi_{q}, \chi_{13}\right)\left[1 / 12 R_{p \pi \eta}^{q} R_{p}^{d} \chi_{13}^{2}-1 / 24 R_{q}^{c} R_{p}^{d} \chi_{13}^{2}\right] \\
& +1 / 24 H_{21}^{F^{\prime}}\left(3, \chi_{13}, \chi_{p}, \chi_{m}, \chi_{13}\right) R_{p}^{d} R_{m n 13}^{v} \chi_{13}^{2}+1 / 48 H_{21}^{F^{\prime}}\left(7, \chi_{13}, \chi_{p}, \chi_{p}, \chi_{13}\right)\left(R_{p}^{d}\right)^{2} \chi_{13}^{2} \\
& +1 / 24 H_{21}^{F^{\prime}}\left(7, \chi_{13}, \chi_{1}, \chi_{3}, \chi_{13}\right) R_{1}^{d} R_{3}^{d} \chi_{13}^{2} \text {. }
\end{aligned}
$$
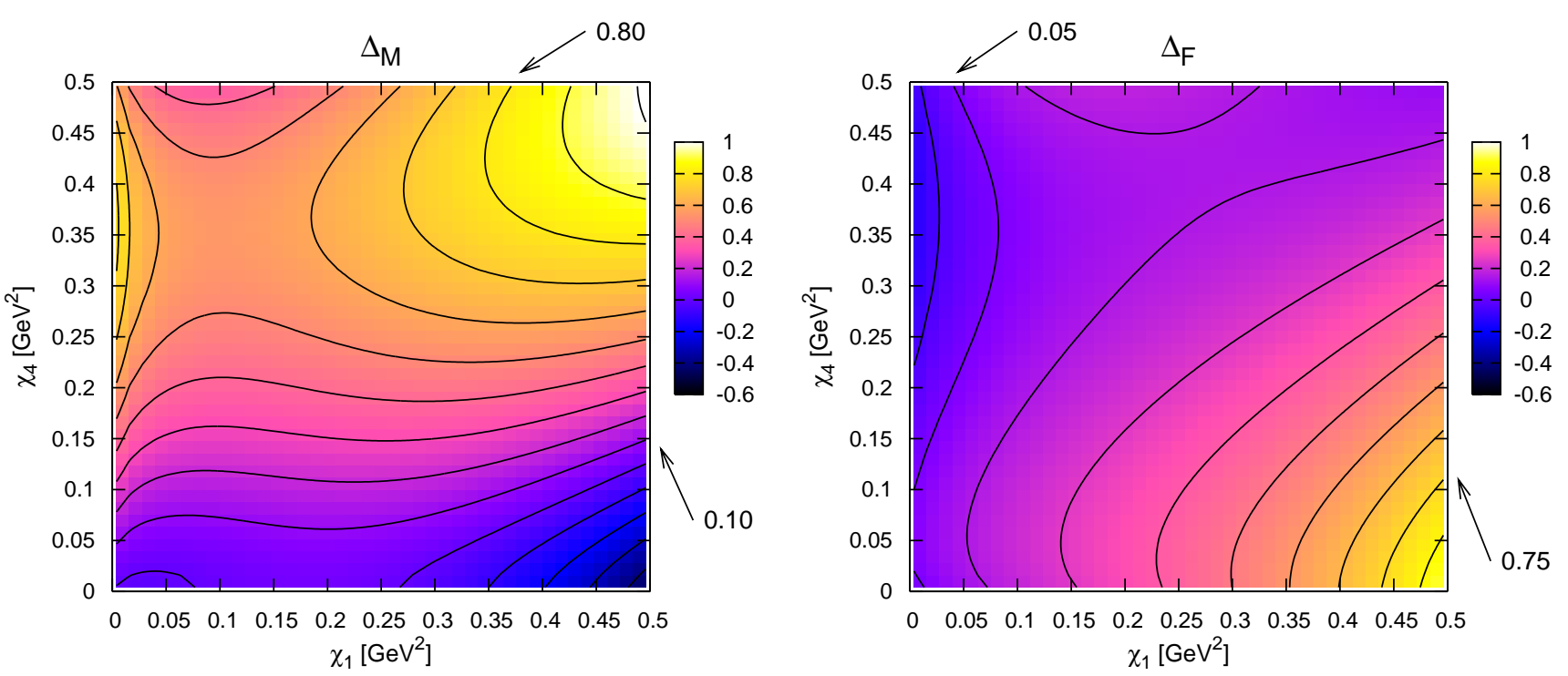

FIG. 3: The relative shifts of the charged pseudoscalar meson mass $\Delta_{M}$ and decay constant $\Delta_{F}$ to NNLO for $d_{\mathrm{val}}=1$ and $d_{\text {sea }}=1$, as a function of the valence and sea-quark masses $\chi_{1}$ and $\chi_{4}$. The quantity plotted represents the sum of the NLO and NNLO shifts, and the difference between two successive contour lines in the plots is 0.10 . The values chosen for the LEC:s correspond to "fit 10" as discussed in the text.

\section{NUMERICAL RESULTS AND DISCUSSION}

\section{A. Checks on the Calculation}

It is mandatory, in such a lengthy and complex calculation, that all possible measures be taken to ensure the correctness of the analytical as well as the numeri- cal results. First of all, the divergence structure and the cancellation of nonlocal divergencies has been found to behave as expected. However, finiteness alone is not a very rigorous check on the end results, as several of the two-loop sunset integrals are convergent because of the appearance of double poles in the propagators. A much more rigorous check is provided by the fact that most 

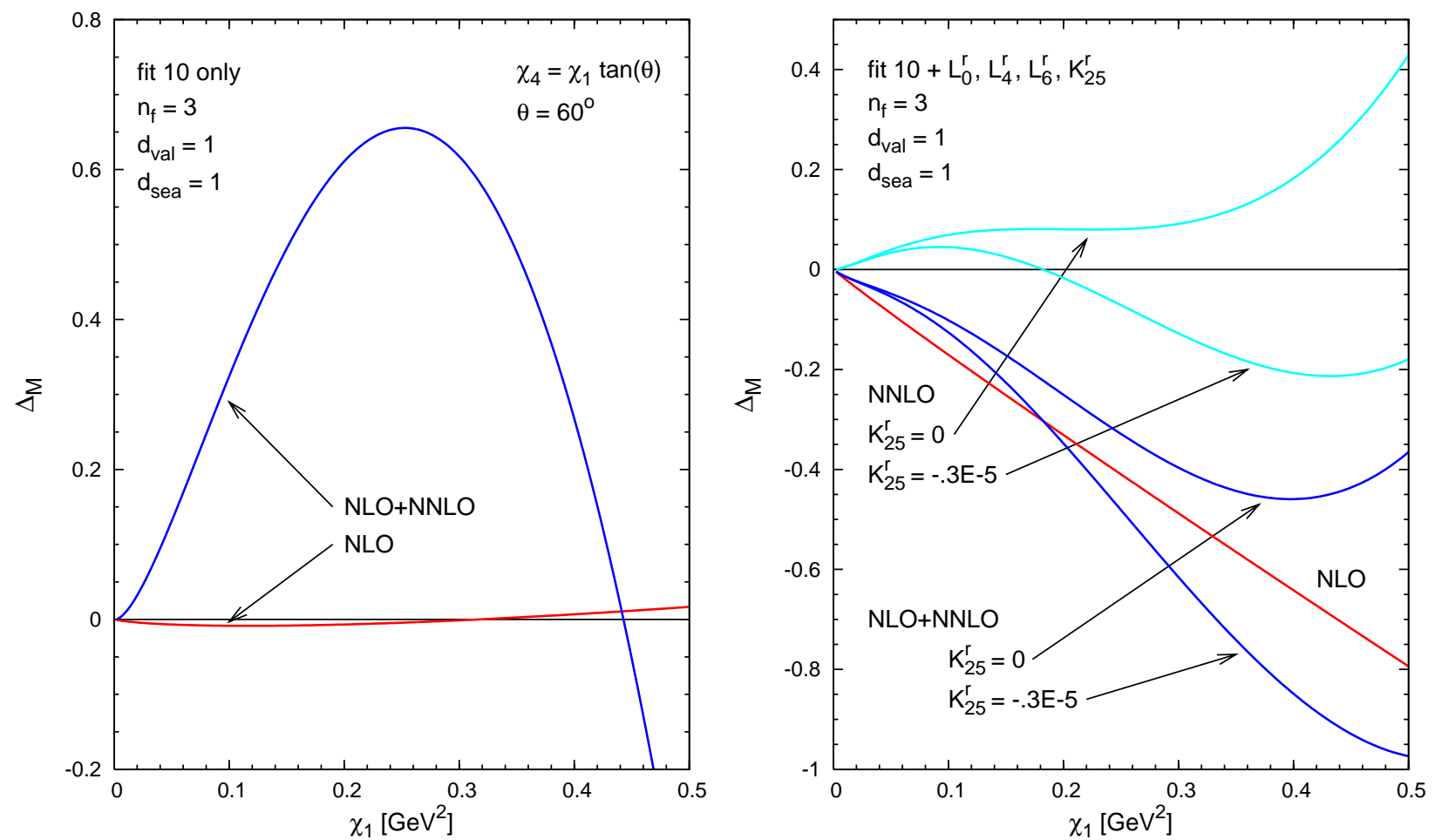

FIG. 4: Comparison of the NLO, NNLO and total NLO+NNLO shifts of the charged meson mass for $d_{\text {val }}=1$ and $d_{\text {sea }}=1$. The left-hand plot shows the NLO and NLO+NNLO results for the set of LEC:s labeled 'fit 10', which has been used for

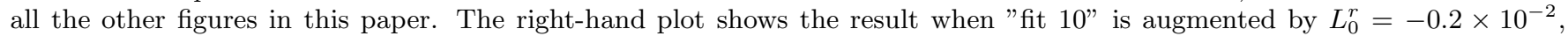
$L_{4}^{r}=0.1 \times 10^{-3}$, and $L_{6}^{r}=-0.1 \times 10^{-3}$. The effects of introducing a nonzero value for the $\mathcal{O}\left(p^{6}\right)$ LEC $K_{25}^{r}$ is also demonstrated for the NNLO and NLO+NNLO results.

parts of the calculation have been performed independently by each one of the authors, such that the computer programs written to handle the symbolic manipulations (mainly using FORM) have been developed independently. At the end of the calculation, and at several intermediate steps during the process, the output from each program has been compared and cross-checked, to make sure that perfect agreement was found. These checks are highly nontrivial, since the form of the (equivalent) outputs is by no means unique. Firstly, the exact appearance of the result depends on the order in which the various symmetries inherent in the Feynman diagrams have been implemented. Secondly, the various loop integrals and propagator residues $R$ satisfy a large number of nontrivial relations, yielding further possibilities to rewrite the result. In the very end, the output from one computer program was simplified and compressed (by more than an order of magnitude) to bring it into a publishable size. Again, that expression was thoroughly checked to determine whether the compactified analytical expressions were still equivalent to the other two versions.

The computer programs for the numerical treatment of the analytical expressions have also been produced in- dependently of each other. Thus the risk for accidental cross-contamination has been minimized as each code was produced in a different programming language ( $\mathrm{f} 77$, f90 and $\mathrm{C++}$ ). All three programs have been found to agree with each other, and the permutation symmetries under exchange of quark masses have been verified up to the numerical precision. As a final check, the general mass cases have been found to converge numerically, as expected, towards the less complex ones when pairs of quark masses become degenerate.

\section{B. Presentation of Numerical Results}

In view of the complexity of the analytical NNLO results in $\mathrm{PQ} \chi \mathrm{PT}$, a graphical presentation of the results is clearly called for. In general, the corrections to the pseudoscalar meson masses and decay constants are real-valued functions of all the sea and valence quark masses. Since, for the nondegenerate cases, this involves the graphical representation of a function which depends on up to five different quark masses, an exhaustive plot is obviously difficult to produce. Except for the most de- 


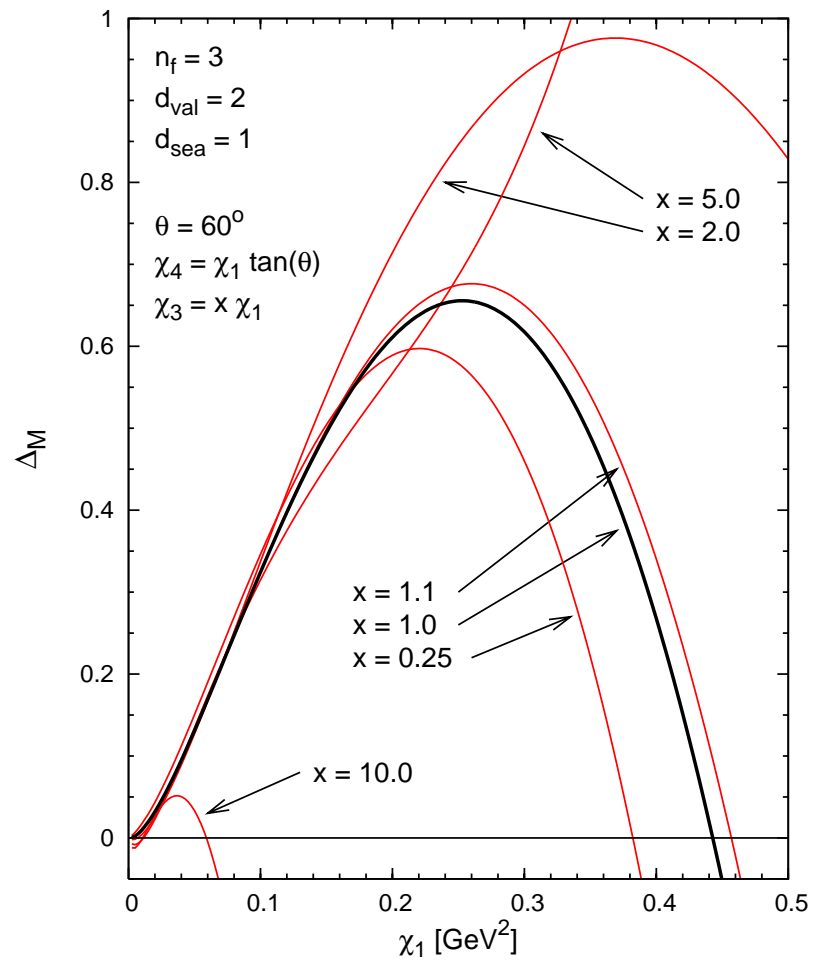

FIG. 5: The combined NLO and NNLO shifts $\Delta_{M}$ of the charged pseudoscalar meson mass, plotted for $d_{\text {val }}=2$ and $d_{\text {sea }}=1$, for $\theta=60^{\circ}$ in the $\chi_{1}-\chi_{4}$ plane, and for the proportionality factors $x=\{0.25,1.1,2.0,5.0,10.0\}$ between the valence quark masses $\chi_{3}$ and $\chi_{1}$.

generate $(1+1)$ mass case, the choice has therefore been to present everything as a function of the valence quark mass $\chi_{1}$ only, by parameterizing the dependence on all other quark masses in terms of $\chi_{1}$. Furthermore, due to the size and complexity of the analytical expressions, their practical usefulness depends highly on the availability of software which can produce numerical output from these expressions in a reliable manner. In view of this, the software which was used for the figures in this paper will be made available in the near future from the website [14].

In addition to the quark mass dependence, the NNLO expressions are also functions of a number of largely unknown LEC:s. In the long run, these LEC:s should of course be determined by a fit of the PQ $\chi \mathrm{PT}$ formulas to Lattice QCD data. At the present time, this is not yet possible, although suitable simulation results should become available in the near future. Therefore, the present work makes use of the LEC:s determined by a fit to experimental data, referred to as "fit 10", which is presented in Ref. 23]. That fit has $F_{0}=87.7 \mathrm{MeV}$ and a renormalization scale of $\mu=770 \mathrm{MeV}$. The NNLO LEC:s $K_{i}^{r}$ and the NLO LEC:s $L_{4}^{r}, L_{6}^{r}$ and $L_{0}^{r}$ were not determined in "fit 10", and they have thus been set to zero for simplicity. However, some results for nonzero values of these LEC:s are presented in Fig. 4. It should be noted that $L_{0}^{r}$ cannot, as discussed earlier in this paper, be determined from experimental data, since it is a distinguishable quantity only in the PQ theory. Some recent results on $L_{4}^{r}$ and $L_{6}^{r}$ have been obtained in Ref. 24], but they have nevertheless been set to zero in most of the plots in this paper, since the present numerics are mainly intended for illustrative purposes.

In the next subsections, the NNLO meson masses and decay constants are presented in terms of the relative shifts $\Delta_{M}$ and $\Delta_{F}$ respectively, which represent the change in the indicated quantity due to the NLO and NNLO contributions. For the pseudoscalar meson mass, $\Delta_{M}$ is defined by

$$
\Delta_{M}=M_{\text {phys }}^{2} / \chi_{i j}-1,
$$

where $\chi_{i j}$ has again been substituted for the lowest order result. The calculation of $\Delta_{M}$ thus involves the numerical evaluation of $M_{\text {phys }}^{2}$ in Eq. (52) to the order of the plot in question. For clarity, it is useful to recall here the definitions

$$
\begin{aligned}
\chi_{i} & =2 B_{0} m_{q i}, \\
\chi_{i j} & =\left(\chi_{i}+\chi_{j}\right) / 2,
\end{aligned}
$$

where $m_{q i}$ denotes the (current) mass of the quark $q_{i}$. In $\chi \mathrm{PT}$, this corresponds to the lowest order mass of a meson composed of a quark and an antiquark, each of mass $m_{q i}$. As an example, the quantity $\chi_{1}$ plotted on the horizontal axes of Figs. 3 and 4 represents the lowest order mass of the valence quark meson $\bar{q}_{1} q_{1}$, and is given by $\chi_{1}=2 B_{0} m_{1}$. Where no confusion can arise, the quantities $\chi_{i}$ are sometimes referred to as "quark masses".

The decay constant shift $\Delta_{F}$ is defined in a similar way, such that

$$
\Delta_{F}=F_{\text {phys }} / F_{0}-1,
$$

where $F_{\text {phys }}$ of Eq. (70) is again evaluated to the desired order. Since the decay constant has been treated in detail in Ref. [10], the numerical analysis of this paper will mainly focus on the pseudoscalar meson masses. The dependence of the results on the sea-quark mass $\chi_{4}$ and the valence quark mass $\chi_{3}$ is parameterized in terms of an angle $\theta$ in the $\chi_{1}-\chi_{4}$ plane and a proportionality factor $x$, according to

$$
\begin{aligned}
& \chi_{4}=\tan \theta \chi_{1}, \\
& \chi_{3}=x \chi_{1},
\end{aligned}
$$

and the deviations of the remaining sea quark masses from $\chi_{4}$ are similarly given by

$$
\begin{aligned}
& \chi_{5}=y \chi_{4}, \\
& \chi_{6}=z \chi_{4} .
\end{aligned}
$$



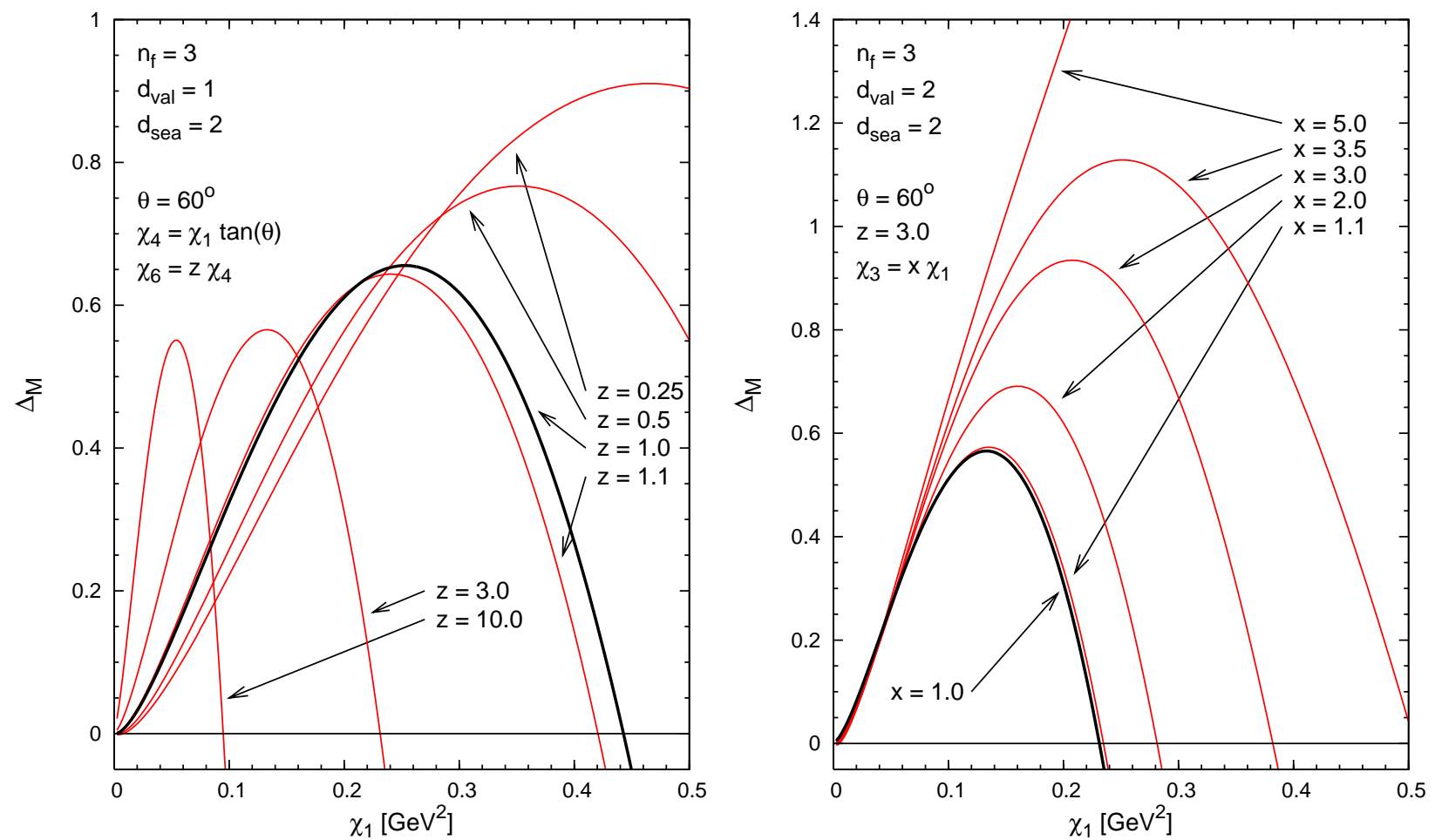

FIG. 6: The combined NLO and NNLO shifts $\Delta_{M}$ of the charged meson mass plotted for $d_{\text {sea }}=2$ and $\theta=60^{\circ}$. The left-hand plot shows the results for $d_{\mathrm{val}}=1$ and a ratio between the sea-quark masses $\chi_{6}$ and $\chi_{4}$ of $z=\{0.25,0.5,1.0,1.1,3.0,10.0\}$. The right-hand plot shows the $d_{\mathrm{val}}=2$ result for $z=3.0$ and a ratio between the valence quark masses $\chi_{3}$ and $\chi_{1}$ of $x=\{1.0,1.1,2.0,3.0,3.5,5.0\}$.

The values of $x, y, z$ and $\theta$ shown in the various plots in this paper have been chosen for convenience, and do not carry any particular significance. A value $\theta>45^{\circ}$ was chosen since the sea-quark masses are then heavier than the valence ones, a situation which is encountered in realistic Lattice QCD simulations. The values for the other quark mass ratios have been picked so as to illustrate a large range of possible quark mass combinations, but they have otherwise been arbitrarily chosen.

The expressions calculated to NNLO for degenerate quark masses should be numerically recoverable as limits of the more general ones, which serves as a useful consistency check. This has been checked for all cases studied, and is explicitly demonstrated for each of the proportionality factors $x, y$ and $z$. For each diagram, one of the plot curves from a more degenerate mass case (the thicker black curve) has been included for comparison. The curve with the new mass ratio, $x, y$ or $z$ equal to 1 shows the more degenerate case and the curve with the ratio equal to 1.1 shows how the more degenerate case is approached.

A naive validity criterion for $\mathrm{PQ} \chi \mathrm{PT}$ is that all masses should satisfy approximately $\chi_{i} \leq 0.3 \mathrm{GeV}^{2}$, but this should naturally be separately checked for each quantity in question. Note that the plots presented in this paper have been extended to include also regions where this constraint is not satisfied. A general study of the convergence of the $\mathrm{PQ} \chi \mathrm{PT}$ expansion up to NNLO is beyond the scope of this paper, as realistic values obtained from Lattice QCD simulations should be used for all LEC:s before any meaningful statements concerning the relative magnitude of the NNLO corrections can be made. The question of convergence then hinges on whether it is possible to describe the Lattice QCD data in such a way that the NNLO correction is of a reasonable magnitude. However, on a qualitative level, the convergence is typically better for the decay constant than for the mass, and the inclusion of nonzero values for the $L_{i}^{r}$ tends to improve the convergence. Nevertheless, it should be kept in mind that these statements are mainly valid for the LEC:s of "fit 10" since a general study of the convergence has not yet been performed.

It should also be noted that an example which demonstrates the effect of variation of the $\mathcal{O}\left(p^{4}\right)$ LEC:s as well as the introduction of nonzero values for the $\mathcal{O}\left(p^{6}\right)$ LEC:s on the convergence of the chiral expansion (in the most degenerate mass case) is shown in Fig. 4 and discussed in Sect. $\mathrm{VC}$ 

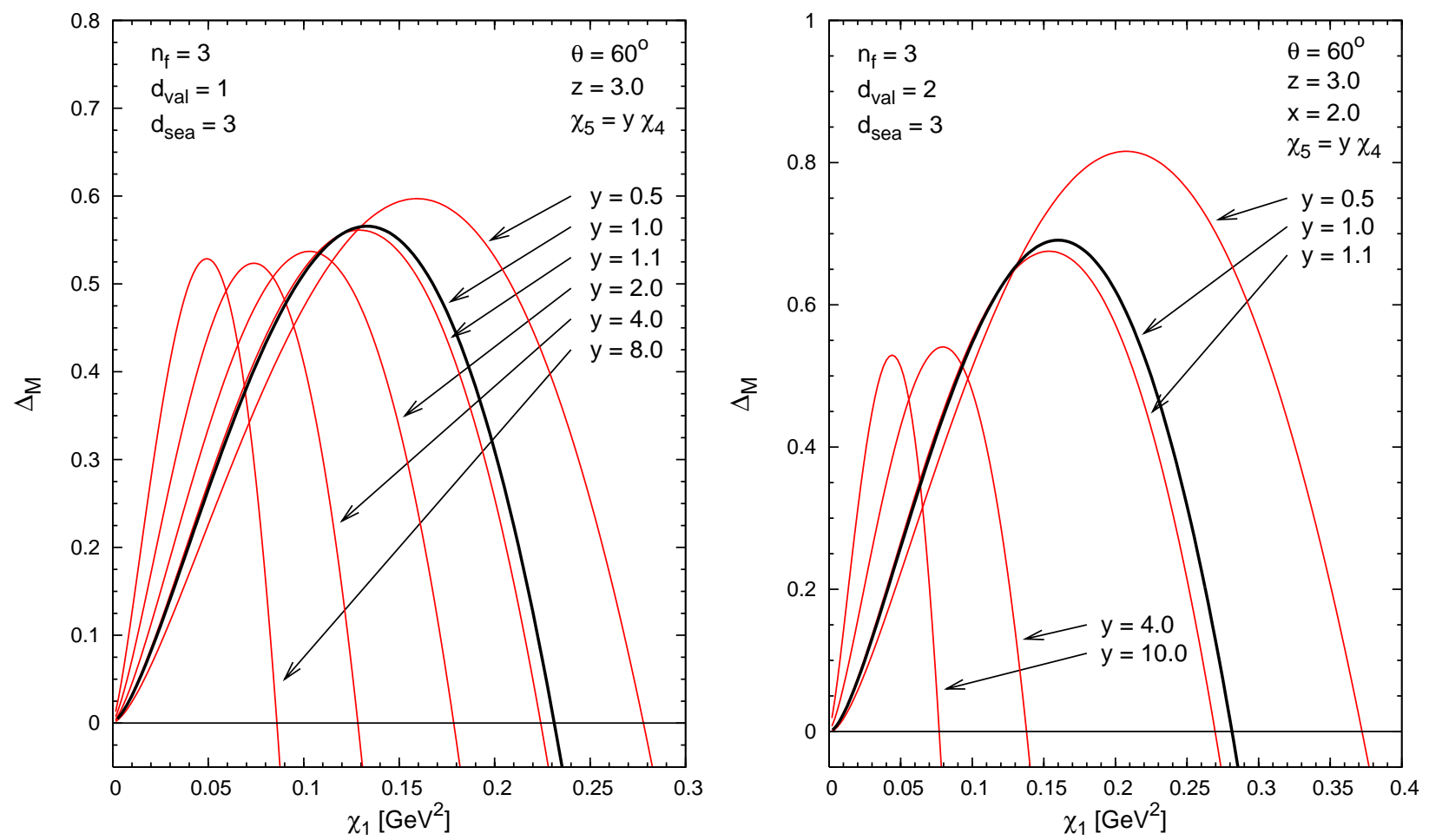

FIG. 7: The combined NLO and NNLO shifts of the charged meson mass, in the left-hand plot for $d_{\text {val }}=1$ and $d_{\text {sea }}=3$, with $\theta=60^{\circ}, z=3.0$ and a ratio $\chi_{5} / \chi_{4}$ of $y=\{0.5,1.0,1.1,2.0,4.0,8.0\}$. The right-hand plot shows the case of $d_{\mathrm{val}}=2$ and $d_{\text {sea }}=3$, with $\theta=60^{\circ}, z=3.0, x=2.0$ and $y=\{0.5,1.0,1.1,4.0,10.0\}$

\section{Numerical Results for $d_{\text {sea }}=1$}

Numerical results for the simplest possible case, where both valence quarks are degenerate, i.e. $x=1$, have already appeared in a number of earlier works. In particular, the case of degenerate sea-quark masses, $y=z=1$, has been considered extensively in Refs. 9] and 10] both for the meson mass and the decay constant, as well as for different choices of LEC:s. In addition to this, Fig. 3 shows a two-dimensional plot of the mass shift $\Delta_{M}$ and the decay constant shift $\Delta_{F}$ over the whole parameter space up to $0.5 \mathrm{GeV}^{2}$. These include thus $\Delta_{M}$ and $\Delta_{F}$ for all values of $\theta$.

An important question in the context of PQ $\chi \mathrm{PT}$ calculations is the issue of convergence of the chiral expansion as a function of the input quark masses. At present, this question cannot be easily answered since the behavior of the NNLO expressions given in this paper depends rather sensitively on the values of the LEC:s. This point is well illustrated by Fig. 4 where the NLO mass shift for $\theta=60^{\circ}, d_{\mathrm{val}}=1$ and $d_{\text {sea }}=1$ has been given explicitly. For the set of LEC:s labeled "fit 10", the NLO result is very small and the total result is completely dominated by the NNLO contribution. For this set of LEC:s, there is obviously no good convergence and the overall corrections are rather large for most values of $\chi_{1}$. Furthermore, the strong curvature exhibited by the NNLO result is not typical of the behavior seen in Lattice QCD simulations. It should be noted that unquenched $\chi \mathrm{PT}$ also shows similar behavior 23] for these values of the LEC:s.

This apparently pathological behavior can be completely changed by appropriately tuning the values of the LEC:s, as shown in Fig. 4 From the results shown in that plot, one can conclude that "fit 10" with small changes is actually completely compatible with excellent convergence of the PQ $\chi \mathrm{PT}$ expansion. In fact, by choosing $L_{0}^{r}=-0.2 \times 10^{-2}, L_{4}^{r}=0.1 \times 10^{-3}$ and $L_{6}^{r}=-0.1 \times 10^{-3}$, the dramatic curvature in the NNLO contribution is straightened out and the near total cancellation in the NLO result is eliminated. The total NLO+NNLO result then lies close to the NLO one even for rather large values of $\chi_{1}$ and $\chi_{4}$. The effects of considering nonzero values of the LEC:s at $\mathcal{O}\left(p^{6}\right)$ can be sizeable as well. The changes that result from the introduction of a naturally sized value of $K_{25}^{r}=-0.3 \times 10^{-5}$ are also shown in Fig. 4] Overall, these changes in the values of the LEC:s yield a nicely convergent chiral expansion and a rather smooth and featureless final result. 


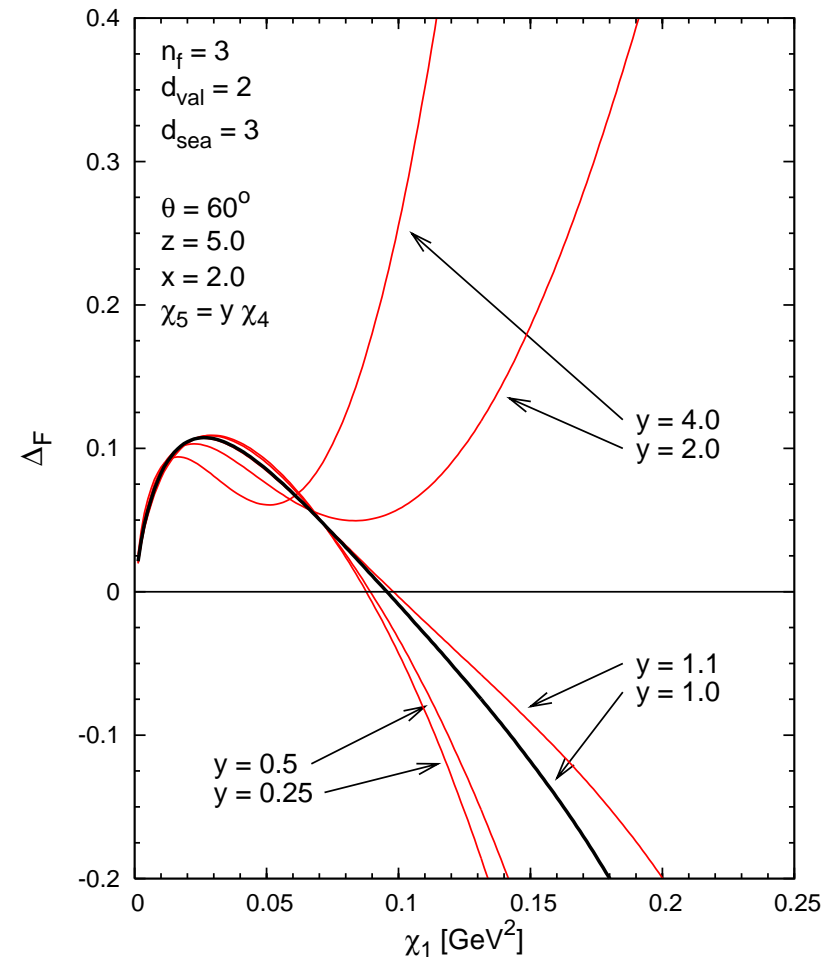

FIG. 8: The combined relative NLO and NNLO shifts of the charged meson decay constant, $\Delta_{F}$, plotted for $d_{\mathrm{val}}=2$ and $d_{\text {sea }}=3$, with $\theta=60^{\circ}, z=5.0, x=2.0$ and $y=$ $\{0.25,0.5,1.1,1,2,4\}$. Note that further plots for smaller values of $d_{\text {val }}$ and $d_{\text {sea }}$ can be found in Ref. 11].

For the case of $d_{\mathrm{val}}=2$ and $d_{\text {sea }}=1$, the mass shift $\Delta_{M}$ has been plotted in Fig. 5 as a function of the input valence quark mass $\chi_{1}$ for several values of the valence quark mass ratio $x$. Note that the shifts $\Delta$ should vanish in the chiral limit, $\chi_{i} \rightarrow 0$, which is obviously satisfied by the plots since the sea-quark masses also vanish for $\chi_{1} \rightarrow 0$. On the other hand, it is apparent from Fig. 3 that the shifts $\Delta$ do not vanish if the valence quark mass is set to zero for a constant value of the sea-quark mass. This effect is due to the quenched chiral logarithms and constitutes a generic feature of the PQ theory. Furthermore, Fig. 5 shows that the results for the $(2+1)$ mass case converge well toward the $(1+1)$ mass case (the thick black curve) in the limit $x \rightarrow 1$.

\section{Numerical Results for $d_{\text {sea }}=2$ and $d_{\text {sea }}=3$}

The expressions for $d_{\text {sea }}=2$ have two sea-quark mass parameters, $\chi_{4}$ and $\chi_{6}$. In the numerical analysis, the latter is given in terms of the former by the ratio $z$ of Eq. (78). As for $d_{\text {sea }}=1$, the sea-quark masses are parameterized with respect to the valence quark mass $\chi_{1}$ by the angle $\theta$, as in Eq. (77). Similarly, for $d_{\text {val }}=2$ the relationship between the valence quark masses $\chi_{1}$ and $\chi_{3}$ is given by the ratio $x$ as defined in Eq. (77). For the case of $d_{\text {sea }}=2$, the behavior of $\Delta_{M}$ as a function of $\chi_{1}$ is illustrated, for different choices of $x$ and $z$, in Fig. 6] As for the previous mass case, all mass shifts vanish in the chiral limit for all cases shown in Fig. 6] In the lefthand plot of Fig. [6] the thick black curve of the mass case $(1+1)$ has been plotted for the same parameters as in Fig. [5] and as expected, the NNLO correction again approaches this curve in the limit $z \rightarrow 1$. In the righthand plot of Fig. [6] the thick black curve corresponds to the mass case $(1+2)$, with $60^{\circ}$ and $z=3.0$. There, the convergence to the more degenerate quark mass configuration is quite fast, as the plot for $x=1.1$ lies very close to that curve.

Finally, the case with $d_{\text {sea }}=3$ depends on all three sea-quark mass parameters $\chi_{4}, \chi_{5}$ and $\chi_{6}$, given in terms of the $\theta$ and $y, z$ defined in Eqs. (77) and (78). In the left-hand plot of Fig. $7 \Delta_{M}$ is shown as a function of $\chi_{1}$ for $d_{\text {val }}=1$ and $d_{\text {sea }}=3$, and in the right-hand plot the case with $d_{\text {val }}=2$ and $d_{\text {sea }}=3$ is shown. As for all previous cases, the mass shifts vanish in the chiral limit. In both plots of Fig. 7 the thick black curves can be found among the lines plotted for $d_{\text {sea }}=2$ in Fig. [6] Again, the convergence to the respective thick black lines is seen to be properly attained when $y \rightarrow 1$. For completeness, a plot of the relative NNLO correction to the decay constant in shown in Fig. 8. Plots for the more degenerate mass cases can be found in Ref. [10.

\section{E. Determination of LECs from Lattice QCD simulations}

As noted in the previous sections, the main motivation for NNLO calculations in $\mathrm{PQ} \chi \mathrm{PT}$ is that such expressions can be used to analyze the results of PQ Lattice QCD simulations in terms of the LEC:s of unquenched QCD. Up to this point, the numerical results presented in this paper have illustrated the quark mass dependence of the NNLO expressions for a given set of LEC:s. In this section, the attention is turned towards finding efficient methods of determining the LEC:s from fits of the NNLO expressions to future Lattice QCD data. It should be kept in mind that the LEC:s denoted by $L_{i}^{r}$ and $K_{i}^{r}$ always refer to the $L_{i}^{r(3 p q)}$ and the $K_{i}^{r(3 p q)}$ of the PQ theory with $n_{\text {sea }}=3$. An equivalent discussion for the case of $n_{\text {sea }}=2$ can be found in Ref. [11].

At NLO, the expressions for the masses and decay constants of the pseudoscalar mesons can be separated into a tree-level contribution, which is a function of the $L_{i}^{r}$, and a remaining part independent of the $L_{i}^{r}$, which involves the chiral logarithms. In the following developments, the average quark mass is denoted by $\bar{\chi}$, where the index denoting the power of the sea quark masses averaged has been dropped for simplicity. Note also the use of the product of lowest order sea-meson masses $\chi_{\pi} \chi_{\eta}$, which was defined in Eq. (28). For the most general case of $d_{\mathrm{val}}=2$ and $d_{\mathrm{sea}}=3$, the dependence on the $L_{i}^{r}$ is then 
proportional to

$$
f_{\mathrm{ct}}^{(4) 23} \sim 3 \bar{\chi} L_{4}^{r}+\chi_{13} L_{5}^{r}
$$

for the decay constant, and

$$
\delta_{\mathrm{ct}}^{(4) 23} \sim 3 \bar{\chi}\left(2 L_{6}^{r}-L_{4}^{r}\right)+\chi_{13}\left(2 L_{8}^{r}-L_{5}^{r}\right)
$$

for the mass 7], from which an overall factor of $\chi_{13}$ has been removed. The expressions for the more degenerate quark mass cases can then be obtained by consideration of the appropriate limits. For the expressions in Eqs. (79) and (80), these limits can be taken straightforwardly, and it can be seen at once that only the simplest $(1+1)$ mass case is required in order to determine all the NLO LEC:s present in the expressions, since one can determine $L_{4}^{r}$ and $L_{5}^{r}$ by fitting Eq. (79) to Lattice QCD data, and then use this knowledge to determine the remaining two constants from Eq. (80). The more complicated cases with nondegenerate quarks are thus redundant for a determination of the LEC:s at NLO.

A similar analysis at NNLO becomes more challenging, not because of the analytical complexity of the NNLO expressions, but rather because the chiral logarithms are no longer independent of the $L_{i}^{r}$. On the other hand, the dependence of the NNLO expressions on the $K_{i}^{r}$ is similar to that of the NLO ones on the $L_{i}^{r}$, with the exception that the $K_{i}^{r}$ parameters at NNLO are slightly more numerous than the $L_{i}^{r}$ at NLO. In all, the $\mathcal{O}\left(p^{6}\right)$ expressions depend on a total of $12 K_{i}^{r}$ parameters, all of which occur in the expression for the meson mass, whereas the expression for the decay constants only depends on five of them. For the decay constants, the expression for $d_{\text {val }}=2$ and $d_{\text {sea }}=3$ can be factorized into the form

$$
\begin{aligned}
f_{\mathrm{ct}}^{(6) 23} \sim & 2 \chi_{13}^{2} K_{19}^{r}-\chi_{1} \chi_{3}\left(K_{19}^{r}-K_{23}^{r}\right) \\
& +3 \bar{\chi} \chi_{13} K_{20}^{r}+9 \bar{\chi}^{2}\left(K_{21}^{r}+K_{22}^{r}\right) \\
& -6 \chi_{\pi} \chi_{\eta} K_{21}^{r}
\end{aligned}
$$

up to an overall numerical factor which has been omitted. From this expression, it is then possible to determine all 5 LEC:s $K_{19}^{r}$ through $K_{23}^{r}$. The $(1+1)$ mass case suffices to determine three combinations of LEC:s from Eq. (81), but in order to separate $K_{19}^{r}$ from $K_{23}^{r}$ at least one case with $d_{\text {val }}=2$ has to be considered, otherwise only the combination $K_{19}^{r}+K_{23}^{r}$ is accessible, as can be readily seen by setting $\chi_{3} \rightarrow \chi_{1}$ in Eq. (81). Similarly, in order to separate $K_{21}^{r}$ from $K_{22}^{r}$, at least one case with $d_{\text {sea }}=2$ has to be considered, since only the combination $K_{21}^{r}+$ $3 K_{22}^{r}$ can be distinguished for completely degenerate seaquarks, i.e. $d_{\text {sea }}=1$. This analysis is identical in form to the one for $\mathrm{PQ} \chi \mathrm{PT}$ with $n_{\text {sea }}=2$ given in Ref. [11]. It is actually possible, by making the replacements $\chi_{13} \rightarrow$ $\chi_{12}, \bar{\chi} \rightarrow 2 / 3 \chi_{34}$ and $\chi_{\pi} \chi_{\eta} \rightarrow \chi_{3} \chi_{4} / 3$ to recover the corresponding expression for $n_{\text {sea }}=2$ from the present one given in Eq. (81).

The dependence of the meson masses on the $K_{i}^{r}$ is somewhat more involved, but the structure in the quark mass combinations is similar to that of Eq. (81). Explicitly, the dependence of the NNLO shift of the pseudoscalar meson mass on the $K_{i}^{r}$ is proportional to

$$
\begin{aligned}
\delta_{\mathrm{ct}}^{(6) 23} \sim & -2 \chi_{13}^{2}\left(K_{17}^{r}+K_{19}^{r}-3 K_{25}^{r}-K_{39}^{r}\right) \\
& +\chi_{1} \chi_{3}\left(K_{19}^{r}-K_{23}^{r}-3 K_{25}^{r}\right) \\
& -6 \bar{\chi} \chi_{13}\left(K_{18}^{r}+K_{20}^{r} / 2-K_{26}^{r}-K_{40}^{r}\right) \\
& -9 \bar{\chi}^{2}\left(K_{21}^{r}+K_{22}^{r}-K_{26}^{r}-3 K_{27}^{r}\right) \\
& +6 \chi_{\pi} \chi_{\eta}\left(K_{21}^{r}-K_{26}^{r}\right),
\end{aligned}
$$

from which a factor of $\chi_{13}$ as well as an overall numerical factor has been removed. As for Eq. (81), the $(1+1)$ mass case here gives access to 3 combinations of LEC:s, plus an additional combination for each of the $(1+2)$ and $(2+1)$ mass cases. In all, 5 additional combinations may be determined from the mass expression, even though the dependencies are slightly more entangled. However, it is clear that simulations with nondegenerate quark masses are needed in order to obtain independent information about as many of the $K_{i}^{r}$ as possible. On the other hand, when one determines the $L_{i}^{r}$ from NNLO fits, it may be convenient to work in the $(1+1)$ mass case only, since it is then still possible to distinguish all of the $L_{i}^{r}$, while the number of distinct combinations of $K_{i}^{r}$ parameters is as low as possible, which makes the fitting somewhat less complicated.

It is also useful to compare the three-flavor result in Eq. (82) with the analogous formula in the two-flavor treatment of Ref. [1]. As for the $\mathcal{O}\left(p^{6}\right)$ terms from the three-flavor decay constant, the terms from the mass expressions can be similarly translated into the expression for the two-flavor case using the replacements mentioned in connection with Eq. (81). Any apparent differences in the numerical prefactors are then only due to the slightly different notational conventions between the present work and that of Ref. 11]. Some additional $L_{i}^{r}$ show up in the NNLO expressions, namely $L_{0}^{r}$ through $L_{3}^{r}$ and $L_{7}^{r}$. It has been noted in Ref. [7] that $L_{7}^{r}$ can be determined from the properties of the double pole of the PQ neutral meson propagators. This quantity has not yet been calculated up to NNLO. The other four $L_{i}^{r}$ are those which are relevant for meson-meson scattering. In the present analysis, their values have been used as input, but they could in principle be separated from the $K_{i}^{r}$ since the terms where they are present depend nonanalytically on the quark masses.

While the number of free parameters in the NNLO expressions which need to be fitted to the Lattice QCD data is certainly large, the above analysis has demonstrated that their number is neither unmanageable nor overwhelming. In particular, the possibility of producing Lattice QCD data for different combinations of sea and valence quark masses should simplify matters considerably. 


\section{CONCLUSIONS}

In this paper, a complete calculation of the mass of a charged pseudoscalar meson to NNLO in PQ $\chi \mathrm{PT}$ has been performed, and explicit analytical formulas of a reasonable length have been given for all relevant degrees of degeneracy in the input quark masses. In addition, the hitherto missing NNLO result with three nondegenerate sea-quarks for the decay constant of a charged pseudoscalar meson has been provided here. These expressions could be brought into a manageable size, since the residues of the PQ propagators in the neutral meson sector satisfy a large number of relations between sums of products of ratios of differences of quark masses. As elaborated in Sect. $\mathrm{VA}$ the results have passed a variety of nontrivial consistency checks, analytical as well as numerical.

In the numerical analysis of the NNLO expressions, various plots for different choices of the input quark masses were presented to indicate the typical size of the corrections. From these plots, it is certainly evident that the total NLO + NNLO corrections may be uncomfortably large, unless the input quark masses assume very small values. However, it is known that the NNLO corrections in unquenched $\chi \mathrm{PT}$ are rather large as well [20, 23], which is especially true for the case of the meson mass. The fact that the partially quenched as well as the unquenched expressions depend sensitively on the values of the $L_{i}^{r}$ as well as the $K_{i}^{r}$ suggests that this problem may be entirely due to the omission of the $K_{i}^{r}$ from the numerical analysis. Thus, once a realistic set of values for the LEC:s is available, the convergence of the expansion up to NNLO should be dramatically improved. Nevertheless, one may readily conclude that the NNLO effects are bound to be nonneglegible for values of the quark masses that are presently used in Lattice QCD simulations.

As yet, no fits to available Lattice QCD data have been attempted, as such a comparison is beyond the scope of this article. It may be noted that an extrapolation of the simulation results to zero lattice spacing and to infinite volume is necessary for a rigorous comparison with the $\mathrm{PQ} \chi \mathrm{PT}$ expressions, but on the other hand it is possible to simply include such effects as extra uncertainties in the determined values of the LEC:s.

\section{Acknowledgments}

The program FORM 3.0 has been used extensively in these calculations 22]. This work is supported by the European Union TMR network, Contract No. HPRN - CT - 2002 - 00311 (EURIDICE) and the EU - Research Infrastructure Activity RII3 - CT - 2004 - 506078 (HadronPhysics). TL acknowledges Martin Savage, Aurel Bulgac and Massimiliano Procura for instructive discussions, and the Mikael Björnberg memorial foundation for a travel grant.
[1] S. Weinberg, Physica A 96, 327 (1979);

[2] J. Gasser and H. Leutwyler, Ann. Phys. 158, 142 (1984); J. Gasser and H. Leutwyler, Nucl. Phys. B 250, 465 (1985).

[3] C. W. Bernard and M. F. L. Golterman, Phys. Rev. D 46, 853 (1992) arXiv:hep-lat/9204007.

[4] S. R. Sharpe, Phys. Rev. D 46, 3146 (1992) arXiv:hep-lat/9205020.

[5] A. Morel, J. Phys. (France) 48, 1111 (1987).

[6] C. W. Bernard and M. F. L. Golterman, Phys. Rev. D 49, 486 (1994) arXiv:hep-lat/9306005.

[7] S. R. Sharpe and N. Shoresh, Phys. Rev. D 62, 094503 (2000) arXiv:hep-lat/0006017.

[8] S. R. Sharpe and N. Shoresh, Phys. Rev. D 64, 114510 (2001) arXiv:hep-lat/0108003.

[9] J. Bijnens, N. Danielsson and T. A. Lähde, Phys. Rev. D 70, 111503 (2004) arXiv:hep-lat/0406017.

[10] J. Bijnens and T. A. Lähde, Phys. Rev. D 71, 094502 (2005) arXiv:hep-lat/0501014.

[11] J. Bijnens and T. A. Lähde, Phys. Rev. D 72, 074502 (2005) arXiv:hep-lat/0506004.

[12] F. Farchioni, I. Montvay and E. Scholz $[q q+q$ Collaboration], Eur. Phys. J. C 37, 197 (2004) arXiv:hep-lat/0403014.

[13] C. Aubin et al. [MILC Collaboration], Phys. Rev. D 70, 114501 (2004) arXiv:hep-lat/0407028.
[14] The analytical formulas may be downloaded via: http://www. thep.lu.se/ bijnens/chpt.html. The programs are available on request from the authors.

[15] S. Scherer, in: Advances in nuclear physics, vol. 27, pp. 277-538 hep-ph/0210398; G. Ecker, hep-ph/0011026 A. Pich, hep-ph/9806303

[16] J. Bijnens, G. Colangelo and G. Ecker, Ann. Phys. 280, 100 (2000) arXiv:hep-ph/9907333.

[17] J. Bijnens, G. Colangelo and G. Ecker, JHEP 9902, 020 (1999) arXiv:hep-ph/9902437.

[18] P. H. Damgaard and K. Splittorff, Phys. Rev. D 62, 054509 (2000) arXiv:hep-lat/0003017.

[19] S. R. Sharpe and R. S. Van de Water, Nucl. Phys. Proc. Suppl. 129 (2004) 245 hep-lat/0308010.

[20] G. Amorós, J. Bijnens and P. Talavera, Nucl. Phys. B 568, 319 (2000) arXiv:hep-ph/9907264.

[21] M. Peskin and D. Schroeder, "An Introduction to quantum field theory," Perseus Books, Reading MA, USA (1995).

[22] J. A. Vermaseren, arXiv:math-ph/0010025

[23] G. Amorós, J. Bijnens and P. Talavera, Nucl. Phys. B 602, 87 (2001) arXiv:hep-ph/0101127.

[24] J. Bijnens, P. Dhonte and P. Talavera, JHEP 0401, 050 (2004) arXiv:hep-ph/0401039, JHEP 0405, 036 (2004) arXiv:hep-ph/0404150. 